\title{
Stoking the Flames of Wellness: An Exploration of Factors that Influence West Virginia Firefighters' Health Behaviors
}

Chelsea B. Wooding

West Virginia University

Follow this and additional works at: https://researchrepository.wvu.edu/etd

\section{Recommended Citation}

Wooding, Chelsea B., "Stoking the Flames of Wellness: An Exploration of Factors that Influence West Virginia Firefighters' Health Behaviors" (2014). Graduate Theses, Dissertations, and Problem Reports. 485. https://researchrepository.wvu.edu/etd/485

This Dissertation is protected by copyright and/or related rights. It has been brought to you by the The Research Repository @ WVU with permission from the rights-holder(s). You are free to use this Dissertation in any way that is permitted by the copyright and related rights legislation that applies to your use. For other uses you must obtain permission from the rights-holder(s) directly, unless additional rights are indicated by a Creative Commons license in the record and/ or on the work itself. This Dissertation has been accepted for inclusion in WVU Graduate Theses, Dissertations, and Problem Reports collection by an authorized administrator of The Research Repository @ WVU.

For more information, please contact researchrepository@mail.wvu.edu. 
Stoking the Flames of Wellness: An Exploration of Factors that Influence West Virginia Firefighters' Health Behaviors

\title{
Chelsea B. Wooding, M.S., M.A.
}

\author{
Dissertation submitted \\ to the College of Physical Activity and Sport Sciences \\ at West Virginia University \\ in partial fulfillment of the requirements for the degree of \\ Doctor of Philosophy in \\ Sport and Exercise Psychology
}

Samuel Zizzi, Ed.D., Chair

Christiaan Abildso, Ph.D., MPH

Malayna Bernstein, Ph.D.

Christine Schimmel, Ed.D.

Jack C. Watson, II, Ph.D.

Department of Sport Sciences

Morgantown, West Virginia

2014

Keywords: Firefighters, Health Behaviors, Physical Activity, Nutrition, Qualitative Copyright 2014 Chelsea Wooding 


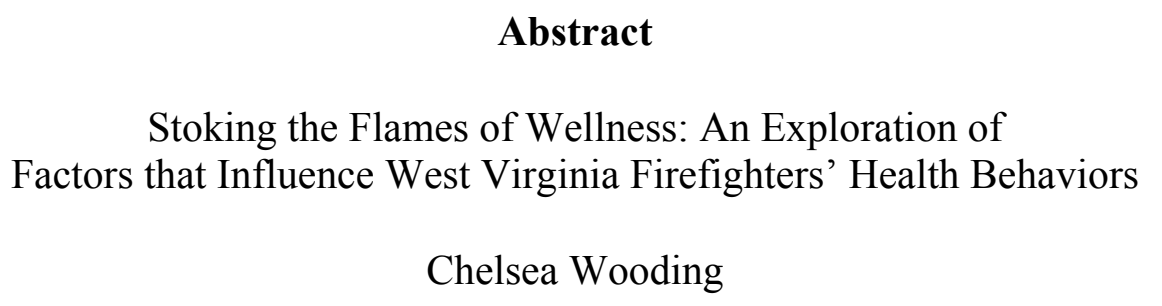

The concern over health and wellness among firefighters in the US has escalated recently due to increasing obesity rates, on-duty cardiovascular event risk, and job stress. Besides unique aspects of the work, a number of other barriers to health and wellness have been identified within the firefighter culture. Using a qualitative approach with multiple sources of data, the current study sought to answer the question, "what impacts the health of firefighters in West Virginia?" Eight focus groups, and Photovoice data from nine participants were inductively analyzed using guidelines from consensual qualitative research. House tours were also done to provide ethnographic data. Seven main factors were identified as impacting firefighters' health in West Virginia: stress, nutrition, general factors, physical activity, sleep, motivation, and job related concerns. Participants also discussed potential solutions to health concerns among firefighters, such as incentive and education programs. Recommendations for future studies, including possible interventions, are discussed. 


\section{Acknowledgements}

To my dissertation chair and advisor, Dr. Sam Zizzi, there are not words to thank you enough. You adopted us all without hesitation, and despite your incredibly busy schedule, you always made sure that we got the time and attention we needed. I know I am not always the easiest person to handle, and your patience and guidance throughout this project and during my time at WVU were invaluable. I truly believe I am a better teacher, practitioner, researcher, and person upon leaving this program, and that is in large part thanks to you. Thank you for everything.

Dr. Watson, your advice about this project and beyond has been incredibly beneficial. Your willingness to invest in your students is inspiring; regardless of how busy you were, you always made time for us. I know the five of us will treasure the time we got to spend with you at job lunches. Please know that the energy you dedicate to all of the students is greatly appreciated.

Malayna, I do not know where this project would be without you. I always enjoyed qualitative research, but your passion for qualitative methods is absolutely contagious! I am so thankful you agreed to be a part of my committee. Thank you for listening to all of my crazy ideas, and helping me figure out how to make them reality.

Christiaan, your advice, and warnings, about travelling through the state helped me mentally prepare for what I was getting into, and your advice about methodology and writing made this project so much stronger. Thank you for being a part of this project, even though you were transitioning to a new department and had so many other things on your plate.

Dr. Schimmel, thank you for all of your advice, and for helping me contact one of the departments. Without you, data collection would have taken even longer. Your support over these last few years, both in and out of the classroom, has been so helpful. Thank you for always encouraging me!

To my wonderful husband, Daron, these last four years might have been harder on you than they were on me in some respects. Thank you for having more strength and patience than I could ever imagine, and thank you for never letting me give up, even though I was not happy with you in those moments. I cannot wait for the next chapter in our journey together. I love you!

To Adrian, Anna, Mac, Megan, Sae-Mi, and Marc, it takes a village to write a dissertation, and I cannot thank you enough for your help. Even at the end of a semester, you all still choose to dedicate some of your truly precious time to help me. I appreciate you all so much. Here's to some good research karma! And to all of my fellow students in the program, thank you for all of the incredible memories and laughs throughout the years - you have a special place in my heart.

And of course, to my mom and family, your unwavering support never ceases to amaze me. Thank you for believing in me when I had no faith left in myself. I would not have gotten through so many years of school without you. And yes, I promise to be done with school now, at least for a little bit! 


\section{Table of Contents}

Introduction 1

Features of Firefighting Linked to Ill Health 1

Volunteer and Career Firefighter Cultures 3

Approaches to Understanding the Phenomenon 3

$\begin{array}{ll}\text { Theoretical Framework } & 6\end{array}$

$\begin{array}{ll}\text { Social Ecological Model } & 6\end{array}$

$\begin{array}{ll}\text { Methods } & 7\end{array}$

$\begin{array}{lr}\text { Sampling Procedures and Participants } & 8\end{array}$

$\begin{array}{ll}\text { Data Collection Procedures } & 9\end{array}$

$\begin{array}{ll}\text { Analysis } & 12\end{array}$

$\begin{array}{ll}\text { Results } & 14\end{array}$

$\begin{array}{ll}\text { Participant Demographics } & 14\end{array}$

$\begin{array}{ll}\text { Focus Groups } & 15\end{array}$

$\begin{array}{ll}\text { House Tours } & 15\end{array}$

$\begin{array}{ll}\text { Photovoice } & 16\end{array}$

$\begin{array}{ll}\text { Overall Results } & 16\end{array}$

$\begin{array}{ll}\text { Discussion } & 32\end{array}$

Potential Interventions $\quad 36$

Recommendations for Future Research 39

$\begin{array}{ll}\text { Strengths } & 40\end{array}$

$\begin{array}{ll}\text { Limitations } & 41\end{array}$

Recommendations for the WV State Firemen's Association 42 
$\begin{array}{ll}\text { References } & 44\end{array}$

Appendices

Appendix A: Extended Review of the Literature 62

Appendix B: Consent Form 106

$\begin{array}{ll}\text { Appendix C: Demographic Survey } & 109\end{array}$

Appendix D: Focus Group Protocol 111

Appendix E: Extended Results 112

Appendix F: Department Maps 156 


\section{List of Tables}

Table 1 - Participant Demographic Information, Means and SD 50

Table 2 - Participant Demographic Information, Frequencies 51

Table 3 - Focus Group Frequency of Themes and Sub-Themes 52

Table 4 - Photovoice Frequency of Themes and Sub-Themes 54

Table 5 - Results in SEM Landscape - Volunteer 56

Table 6 - Results in SEM Landscape - Career 58 


\section{List of Figures}

Figure 1 - SEM Landscape - Volunteer Photos 60

Figure 2 - SEM Landscape - Career Photos $\quad 61$ 


\section{Introduction}

Images of firefighters in popular media tend to portray strong, healthy people, and for good reason. It is comforting to know that those members of society whose job is to take care of and rescue us are strong and healthy enough to successfully perform their duties. The reality, however, is that the health disparities facing the larger American population are plaguing the fire service as well. A range of factors has led to major concerns over the health and well being of firefighters. Research has documented increasing obesity rates, on-duty cardiovascular event risk, and job stress (Durand et al., 2011). In a recent sample of 200 firefighters from five suburban fire departments, more than $50 \%$ qualified as overweight (defined as a body mass index, or BMI, of greater than 25; Durand et al., 2011). Another study using 527 male career firefighters from 10 departments in Kansas and Missouri reported 38\% of the sample was obese (BMI > 30; Byczek, Walton, Conrad, Reichelt, \& Samo, 2004). Obesity among firefighters is important, because the negative association between elevated BMI and health status has been well established (Kales et al., 1999). In fact sudden cardiac death is the number one cause of onduty death among firefighters (Fahy, LeBlanc, \& Molis, 2012). From 2007 to 2011 , 41\% of onduty deaths were caused by sudden cardiac arrest.

\section{Features of Firefighting Linked to Ill Health}

Firefighting is a high stress job (Roberts, O’Dea, Boyce, \& Mannix, 2002). In the course of firefighters' work, they fear for their own personal safety and that of fellow fire fighters, as well as the general public, whose safety is their primary responsibility (Williford, Duey, Olson, Howard, \& Wang, 1999). Research has shown that maintaining minimum levels of fitness helps protects firefighters against the deleterious effects of stress (Guidotti, 1992).

Unique among other physically demanding jobs, firefighters experience occasional peaks 
of strenuous work amidst extended periods of inactivity (Guidotti, 1992). Long phases of free time are often filled with sedentary behavior, a factor related to an increased risk of cardiovascular disease (CVD; Tremblay, Colley, Saunders, Healy, \& Owen, 2010). A range of other contextual factors compounds this already dangerous combination of high stress activity within a sedentary lifestyle for firefighters. First, minimum fitness standards for firefighting have not been established (Durand et al., 2011; Rhea, Alvar, \& Gray, 2004). Similarly, no national mandatory fitness standards are in place for firefighters (Kales, Soteriades, Christophi, \& Christiani, 2007). This lack of standards leaves each fire department responsible to set its own expectations regarding firefighters' fitness levels, causing great variability in fitness levels between firehouses (Byczek et al., 2004). Without clear expectations of an ideal for firefighter fitness, it is difficult for members of the fire service to know what types of exercise are most appropriate or helpful (Jahnke, Poston, Jitnarin \& Haddock, 2012). Furthermore, food options available in the surrounding community and within each firehouse are often a barrier to health, as firefighters report that unhealthy choices, such as donuts and pizza, are readily available and too tempting to resist (Frattaroli et al., 2012).

In addition to unhealthy eating and unclear fitness standards, disrupted and irregular sleep also exacerbates struggles in maintaining health, as firefighters are never sure when they might receive a call (Jahnke et al., 2012). Research has shown short durations of sleep to be significantly associated with obesity in adults; a significant and consistent negative association exists between BMI and hours of sleep (Cappuccio et al., 2008). Unfortunately, a vicious cycle is created in that less sleep is associated with an increase in BMI, and obesity increases the odds of being a short sleeper (Cappuccio et al., 2008). More seriously, an in-depth meta-analysis of research examining the effects of sleep showed that short duration of sleep (defined as anywhere 
from less than five to seven hours of sleep per night) was associated with greater risk of death, findings that remained consistent regardless of age, gender, socioeconomic status, and definition of short duration of sleep (Cappuccio, D’Elia, Strazzullo, \& Miller, 2010).

\section{Volunteer and Career Firefighter Cultures}

Most of the research on firefighters to date has focused on career firefighters, yet volunteers represent the majority of active firefighters. In 2011, of approximately 1.1 million firefighters working in the United States, 69\% were VFFs, while 31\% were CFFs (NFPA, 2013). There are important differences in the experiences of each community that suggest a more nuanced look at the cultures.

First, the two communities are not identical in their health profiles. The rates of being overweight and obese among VFFs are as high as $78.4 \%$ and $43.2 \%$ respectively, rates similar to, or slightly higher than, CFFs at 79.5\% and 33.5\% respectively (Poston et al., 2011). Additionally, the context of their work differs. Volunteer firefighters (VFFs) face additional challenges that could make maintaining health and wellness harder than career firefighters (CFFs). First, VFFs must balance working a full-time job while also working for the fire department (Frattaroli et al., 2012), making time for exercise and healthy eating less available. Additionally, volunteer fire departments often have fewer financial resources, so the cost of incorporating healthier foods or exercise equipment at fire stations might be a barrier (Scanlon \& Ablah, 2008). Clearly, even though there are many shared issues between the two communities, there are significant differences worth attending to.

\section{Approaches to Understanding the Phenomenon}

Until recently, most work examining firefighters' health was quantitative, gathering mass amounts of often decontextualized information about health outcomes or status, such as number 
of deaths or statistics on aerobic capacity. Whereas this data was helpful in painting the landscape in broad brushstrokes and alerting stakeholders to the major concerns within the community, this research does not answer questions about the complex contextual factors that might contribute to firefighters' poor health, nor does it help us understand the relationship between those factors and health. In other words, how does the context of firefighters' work impact their health? Answers to this question will allow stakeholders to develop interventions that are responsive the actual needs of firefighters.

Recently, two research programs started the work of understanding these health factors within the larger context of firefighters' everyday experiences, from the firefighters' perspective. Jahnke and colleagues explored firefighters' perscpectives about increased risk for injury and disease within the fire service (2012). Focus groups were used to learn more about the perceptions, attitudes, and opinions of 332 career firefighters, officers, chiefs, and other fire personnel across 28 departments from western, central, and eastern regions of the United States. These CFFs identified the lack of national standards for firefighter health, lack of departmental mandates, and lack of financial support for health and wellness as major barriers to health behavior (Jahnke et al., 2012). Additionally, firefighters claimed that departments gave limited attention to nutrition (Jahnke et al., 2012). Although participants showed an awareness of the negative health implications related to firefighting, they identified social norms as a major barrier to change (Jahnke et al., 2012).

A related study, conducted by Frattaroli and colleageus, sought to explore the CVD risk among the fire service to develop an intervention based on firefighters' feedback (2012). The researchers used information from 98 volunteer firefighters in the Maryland area across 15 focus groups to inform their intervention. Overall, firefighters admitted to a lack of physical activity 
and nutritious eating. During the focus groups, VFFs discussed barriers to health and wellness within the fire service, including the demanding nature of firefighting and the stress they faced (Frattaroli et al., 2012). Participants focused much of their discussion on their stuggle finding healthy, affordable, quick foods, but firefighters were open to improving their food environment. Even though participants identified personal motivation as the most important factor in increasing physical activity, they were unable to offer concrete suggestions on how to effectively intervene. Firefighters also discussed an inability to prioritize health in modern life, and time limitations from balancing their employment with serving at the fire house (Frattaroli et al., 2012).

Although Jahnke and Frattaroli's research teams have contributed valuable information to the existing literature by providing firefighters' perspectives and focusing on how context is related to outcomes, the studies are not without limitations. For instance, the samples consisted of only CFFs or VFFs, so the findings might not generalize to the other firefighting community. Additionally, information about the rural or urban nature of the department locations was not provided in the previous studies, so it is unclear how variables that are specifically related to rural or suburban locations might factor into the results. Finally, the researchers relied almost exclusively on focus groups for data collection, which inherently have some limitations such as groupthink (Janis, 1972). Moreover, single focus groups provide a snapshot of a group's discussion at a particular moment in time and cannot capture how thinking might evolve or change across different contexts or moments in time. Additionally, in these studies, participants were not provided an opportunity to share their experiences outside the department. The current project, building from these previous studies, attempts to capture a larger picture of firefighter health beyond a single time or space. 


\section{Theoretical Framework}

\section{Social Ecological Model}

Health promotion research began shifting its focus from individual-focused analysis of health, to more environmental-focused strategies in the 1980s (Stokols, 1996). Now, ecological models are more commonplace in the public health field (Golden \& Earp, 2012). Researchers use the social ecological model (SEM) to understand myriad factors that influence health behavior. Social ecological models are helpful in public health research because they "offer a theoretical framework for understanding the dynamic interplay among persons, groups, and their sociophysical milieus" (Stokols, 1996, p. 283). In other words, according to SEM, behavior is dictated by multiple levels of influence (Sallis et al., 2008). Additionally, SEM is a unique approach to learning about various aspects of health because it highlights the interdependence of personal and situational factors rather than focusing on only one factor such as environmental or biological characteristics (Stokols, 1992, 1996).

Bronfenbrenner developed the "ecology of human movement," which suggested new ways to study human development (1977, p. 514). Bronfenbrenner emphasized the interaction between a person and their environment, made up of four successive levels: microsystems (e.g., home, workplace), mesosystems (interactions between microsystems), exosystem (e.g., neighborhoods, schools), and macrosystem (legislation, media, cultures). Although each individual level is important, the key in SEM is how the multiple levels interact to influence behavior.

To date, limited research has explored the culture of firefighting through the SEM lens to gain a better understanding of health behaviors. One exception, conducted by Staley and colleagues, used SEM as a conceptual framework to explore multi-level influences that might 
explain the limitations of past interventions attempting to help firefighters become more fit (2011). The authors identified various facilitators of, and barriers to, fitness at the intrapersonal (e.g., beliefs, motivation, nutrition), interpersonal (e.g., crew dependability, social cohesiveness, crew and captain norms), and organizational (e.g., work environment factors, participation strategies, management support or lack of support) levels. Even though the findings provide support for using SEM within the firefighter culture, participants in this study were all CFFs at only four departments in the southeastern United States (Staley et al., 2011). Consequently, the generalizability of the findings could be limited to firefighters in other regions of the country, or to volunteer firefighters. It is important for researchers to continue using SEM as a guide for understanding multi-level factors that might influence firefighters in other parts of the United States.

The purpose of the study was to gain a better understanding of health behaviors among career and volunteer firefighters in an area of the United States that does not appear to have been examined in previous research. Although we used SEM as a guiding framework for the current study, we also wanted it to be exploratory, because we recognized that we might not know the important influences on health across firefighters' life ecologies. For this reason, our instruments for inquiry needed to be open-ended and participant-driven, so that participants could draw for us the meaningful areas of influence within their individual ecologies.

\section{Methods}

Our goal for the current study was to learn more about the perspectives of career and volunteer firefighters with regards to health and wellness. We used a variety of data collection strategies with both career and volunteer firefighters in different regions of a primarily rural state to continue the ongoing conversation about firefighter health. 


\section{Sampling Procedures and Participants}

Purposive and snowball sampling were used to identify and recruit career and volunteer fire departments in West Virginia. Specifically, multistage sampling was used to first identify career fire departments throughout the state, and then match volunteer fire departments within the same county. Ultimately, four focus groups were conducted at career departments and four focus groups were conducted at volunteer departments. No two volunteer or career departments were in the same county, to ensure that multiple areas of the state were represented in the focus groups. Additionally, among the departments selected, each of the four levels of Rural-Urban Commuting Area (RUCA) Codes (i.e., urban, large rural, small rural, and isolated rural; Rural Health Research Center, 2013) were represented in both volunteer and career departments. Our goal was to recruit a wide range of participants that would include firefighters in both rural and urban settings, representing differences that have not yet been explored in research.

To gain access to the often close and familiar world of firefighters, the lead researcher contacted the 26 career fire departments in WV until one in each level of RUCA codes agreed to take part in the study. Then, volunteer departments in those same counties were contacted until one in each county agreed to participate. Recruiting proved challenging because a number of career departments were difficult to contact, and others $(n=3$ career departments) were unable to participate because of low numbers of firefighters within their department. We attempted to gather a group of six to ten firefighters at each department based on minimum recommendations of previous literature (Chrzanowska, 2002); however, because of size limitations of departments in more rural areas of the state, a lower minimum of four participants was set (Marshall \& Rossman, 2006), and one focus group was limited to only three participants. We recognize that this limits the generalizability of our findings, yet we feel that even with our smaller numbers, 
the range and diversity of our participant sample extends current work in the field.

\section{Data Collection Procedures}

We followed a similar data collection protocol at each department. Upon arrival at the department, we asked to go on a house tour, both to build rapport with the group, as well as to see the full context in which the firefighters work on a daily basis. The lead researcher facilitated the focus group interviews after the house tours. Finally, participants were invited to participate in a final portion of the study, Photovoice, to provide further information on factors that influence their health. Before data collection began, the lead researcher wrote a bracketing statement based on guidelines provided by Tufford and Newman (2010) to identify her biases and assumptions.

House tour. Before each focus group, the lead researcher requested a tour of the firehouse with the chief or other lead person on duty to learn more about the setting in which the firefighters work. Other firefighters who were present were also invited to join the tour. Tours provided an opportunity for the researcher to establish rapport with the participants before the focus groups and to position the firefighters as the experts within their own professional environment. During the tour, the lead researcher created an outline for a map of each firehouse that captured the position and composition of each room, and opportunities for, along with barriers to, health behaviors. Together with the focus group data, these maps helped paint the complex picture of how each firehouse promotes and discourages health behavior, in both policy and built environment. Additionally, though the discussion that took place during the house tour was not transcribed or formally analyzed, walking tours provided informal data about ways in which people relate to certain spaces and generated place-specific information which was helpful in providing context to the focus group discussions (Evans \& Jones, 2011). Tours lasted 
anywhere from ten to 40 minutes, and six of the eight tours were led by the chief, and two tours were led by firefighters who held leadership positions at the department. During the tours, we requested to see places where the firefighters eat, drink, or engage in active or sedentary behaviors. Information gathered from the house tours and maps of each department highlighted influences of health behaviors at the microsystem of Bronfenbrenner's (1977) Social Ecological Model of health behavior.

Focus Group Procedures. One pilot focus group was conducted at a volunteer fire department in the local community to ensure that the focus group prompt was effective and to help refine the facilitator's moderating skills. Upon refining the protocol and her approach, the lead researcher conducted the eight focus group interviews in the study.

At the beginning of each focus group, participants filled out a basic demographic questionnaire that asked for each participant's age, height, weight, gender, years as a firefighter, and information on health conditions such as diabetes, heart disease, and cholesterol. Information gathered from the demographic questionnaire was kept entirely anonymous and was not connected back to focus group recordings. It was important to gather survey data because it provides context for the sample of firefighters who participated in the study, and gives an opportunity to learn more about the demographic makeup of rural career and volunteer firefighters.

The start of the focus group included time for introductions and rapport building in an attempt to build trust and comfort between the participants and interviewer. The primary researcher then asked one open-ended prompt, "What impacts your health as a firefighter in West Virginia?" The prompt was purposefully broad, to allow participants to direct the course of the discussion and to engage with those topics only most meaningful to them. After the initial 
question, probing questions were used to provide clarification, elaboration, and further exploration of other components of physical activity and nutrition of firefighters in WV. Such questions addressed barriers to and supports of health behavior, and changes that could be made to encourage healthy behavior among firefighters. When the focus group concluded, participants had an opportunity to ask questions, as well as expand on their answers if they chose. All focus groups were audio recorded for accuracy (Creswell, 2007).

Besides the lead researcher, a second researcher was also in the room managing the recording devices, ensuring protocol was followed, and taking notes on which participant was speaking when. On average, focus groups lasted about an hour, with the shortest lasting 43 minutes and the longest lasting 61 minutes. Any interruption, such as a call or alarm, was noted. During one focus group, a call came in requiring two participants to leave, and calls came in during another four of the eight focus groups, but the firefighters did not need to respond because the call was for a different department or service (i.e., EMS).

Photovoice. Photovoice is a data-collection technique that allows participants an opportunity to describe features of their community by using cameras to document specific images reflecting their realities (Wang \& Redwood-Jones, 2001). Upon the conclusion of each focus group, participants were invited to partake in a second part of the research study to learn more about individual experiences. Nine total participants opted-in to this portion of the study (seven volunteer, two career), falling within the recommended sample size of seven to 10 participants for Photovoice (Wang, 1999). Photovoice was an important addition to the methodological design because it provided an opportunity for participants to break away from the focus group narrative and represent their own individual experiences. In addition, Photovoice allowed for observation of firefighters' everyday practices on their time, without needing a full 
ethnography, which was beyond the scope of this project. Furthermore, Photovoice allowed participants to show and describe meaningful spaces outside of the firehouse, further expanding the researchers' understanding of the multiple ecological systems at play in the participants' health landscape.

Firefighters who chose to participate in this portion of the study were asked to "take pictures of factors in their daily lives that impact their health" for one week. In addition to the picture, participants were also asked to provide a brief description (one to two sentences) of the photo and how it influences their health. Seven of the nine firefighters that participated in Photovoice had a smartphone with camera and SMS capabilities, and sent photos with descriptions directly to the primary researcher via text message; two participants used a tablet with photo capabilities to take pictures and then send them via e-mail. Participants were given the option to receive text reminders each morning with the prompt originally provided; six firefighters chose to receive those reminders.

\section{Analysis}

The primary researcher transcribed the focus groups verbatim, and each was independently reviewed to verify their accuracy by a third party who was not at the focus groups. Transcripts were then analyzed in a three-person team using Consensual Qualitative Research (CQR), as outlined by Hill (2012). We chose CQR as a guide for the qualitative analysis because its use is becoming more popular in fields such as health psychology and sociology, and it provides an opportunity to understand each participant's narrative within the broader environmental context (Hill, 2012). Additionally, we did not want to limit the interpretation and analysis to just one researcher.

Once themes were identified within each individual focus group, the frequency of 
mentions for each theme were tallied and compared across focus groups to determine if any differences existed between type of department (career versus volunteer) or setting (urban, large rural, small rural, isolated rural). Using MANOVAs, department type and RUCA code were independent variables, and frequencies of the various themes were dependent variables.

To increase the trustworthiness and consistency of the data, a breakdown of domains and core ideas, including descriptions, were sent to the chief of each department to give the firefighters an opportunity for member checking, to ensure the validity and accuracy of the analysis (Kvale \& Brinkmann, 2009). Additionally, the final analysis was reviewed by an auditor, a researcher trained in qualitative research who was not part of the three-person research team, to provide a fresh perspective in checking the validity of the analysis (Hill, 2012). Using the three-person research team to reach consensus also balances subjectivity and reflexivity, adding to the trustworthiness of the analysis.

Pictures from the Photovoice portion of the study were also inductively analyzed based on the themes represented with relation to health behaviors. Both pictures and explanations were considered when coding and thematizing the Photovoice data. Analysis of the photos was done by the lead researcher and a secondary researcher with training in qualitative methodology who was not part of the three-person team analyzing the focus groups, to ensure there would be no bias in interpreting the pictures and their explanations. Although a tenant of pure Photovoice protocol is to share photographs with policy makers, to ensure confidentiality, the pictures received were not shared with anyone outside the research team. Additionally, Photovoice analysis also can include a "town hall" sort of forum for participants to further discuss their pictures and experiences. Again, to maintain confidentiality, this portion of traditional Photovoice analysis was not used. 


\section{Results}

In this section, we provide an outline of the general outcomes from each data source, and then we discuss the themes that emerged across these data sources in detail.

\section{Participant Demographics}

Survey data provided a general picture of the firefighter community in West Virginia. A total of 58 participants contributed to the eight focus groups; 27 career firefighters and 31 volunteer firefighters. The average age of participants was 38.79 years old; the youngest participant was 19 years old and the oldest was 66 years old. Four out of the 58 participants were women. Overall, $69 \%$ of the participants were married, and all 58 participants were white. About $26 \%$ of participants reported their annual household income was between $\$ 35,000$ and $\$ 49,999$, which would place them near the median household income in West Virginia from 2007 to 2011 (US Census Bureau, 2012). However, $48.3 \%$ of participants reported making $\$ 50,000$ or more per year. With regards to education, 36.2\% reported being a high school graduate, and $43.1 \%$ reported completing some college or technical school. Overall, 79.3\% of participants held a high school diploma, a percent close to United States Census Bureau data from West Virginia. Finally, when asked about seven different health risks from the BRFSS, 15.5\% of participants have been told by a doctor that they have high blood pressure, 19.0\% have high cholesterol, and $8.6 \%$ have diabetes.

On average, participants had been firefighting at their current department for 14 years. The shortest time a participant had been firefighting was just under a year, and the longest time was 45 years. There was no significant difference between career and volunteer firefighters in the total amount of years served firefighting, firefighting in West Virginia, or firefighting at their current department; nor was there any difference in the years served based on setting (i.e., urban, 
large rural, small rural, and isolated rural).

The average BMI for the participants was $28.78 \mathrm{~kg} / \mathrm{m}^{2}( \pm 4.66)$, with a low of $20.4 \mathrm{~kg} / \mathrm{m}^{2}$ and a high of $44.9 \mathrm{~kg} / \mathrm{m}^{2}$. The BMI of VFFs was slightly higher than CFFs at $29.47 \mathrm{~kg} / \mathrm{m}^{2}( \pm$ $5.22)$ versus $27.99 \mathrm{~kg} / \mathrm{m}^{2}( \pm 3.85)$ respectively. There were no significant differences in BMI between different settings, but those participants in isolated rural settings had an average BMI slightly higher than other settings $\left(30.63 \mathrm{~kg} / \mathrm{m}^{2}\right.$ for isolated rural, $28.85 \mathrm{~kg} / \mathrm{m}^{2}$ for small rural, $28.24 \mathrm{~kg} / \mathrm{m}^{2}$ for large rural, and $28.01 \mathrm{~kg} / \mathrm{m}^{2}$ for urban settings). For a summary of participant demographics, see Tables 1 and 2.

\section{Focus Groups}

From the focus group data, six main factors were identified as impacting the health of West Virginia firefighters: stress, nutrition, general factors impacting heath, physical activity, sleep, and motivation. Each of these factors was discussed by at least seven of the eight focus groups and was brought up multiple times within each focus group. Participants also shared their insight about potential solutions for the health and obesity problem facing the fire service. For a summary of focus group results, see Table 3 .

\section{House Tours}

The house tours proved informative for a variety of reasons. First, spending time with the chief and firefighters in a more relaxed setting touring a space in which they are comfortable helped build rapport. Additionally, conversations during the house tours proved invaluable in providing context for the focus group discussions. For instance, during one of the tours, a firefighter brought up the various grants available to fire departments. However, they were quick to explain that their firehouse rarely qualified for such grants because many grants are directed toward larger departments in metropolitan areas, or very small departments in rural areas. In 
other words, there is a subset of departments that are unable to benefit from the numerous grants available for funding because they do not qualify based on their size. Knowing this information about the limitations of the grants provided better understanding when firefighters discussed budgetary and financial concerns.

After touring and mapping the eight firehouses that were visited, clear discrepancies appeared. Specifically, CFFs had access to exercise equipment at their department while VFFs did not have exercise equipment within their firehouse. CFFs also have common rooms with large screen televisions and recliners, which could act as a barrier to physical activity. All departments had a large kitchen, fully stocked with various cooking equipment at the firehouse.

\section{Photovoice}

Nine participants took part in the Photovoice portion of the study; seven volunteer and two career firefighters. Between the nine participants, a total of 55 pictures were received. Participants sent an average of six photos, with some participants sending as few as one, and as many as 12 during the seven-day time frame. Three main themes emerged from the analysis of the photographs and descriptions: nutrition, physical activity, and other miscellaneous factors. For a summary of Photovoice results, see Table 4.

\section{Overall Results}

Stress. Stress was the most frequently discussed topic by participants during the focus groups (149 total mentions), who spoke about many different sources of stress (81 mentions), as well as various coping strategies used to counter that stress (64 mentions). Three of the focus groups also shared how stress affects their overall health.

The three most frequently discussed sources of stress for participants were seeing bad things on a call (nine mentions in three focus groups), the uncertainty of calls (nine mentions in 
four focus groups), and knowing the victim of an emergency (four mentions in three focus groups). Participants shared that seeing difficult sights on a call can stay with them for years after that emergency is resolved, causing stress both in the present and for years to come. One participant simply stated, "We've all seen things we shouldn't see." Another participant elaborated,

I've heard some people that's been firemen for 10, 15, 20, 30 years say you know, like, there's that one call that's gonna stay with them the rest of their life. It's not just gonna be a period of time, it's forever. I mean, that's something they're gonna think about. Additionally, the uncertainty of each call was a frequently discussed source of stress. When a call comes in, firefighters receive a general description of the emergency; however, participants explained that many times they will reach the site of the call and it will be far worse than originally described. Other less significant sources of stress included the uncertainty of their schedules, the responsibilities of their job, and the emotional stress they experience.

Finally, three focus groups expressed concern when a call came in because there was a high likelihood that they would know the victim of the emergency. The pressure to save someone is already high as a firefighter, but when the victim is a personal friend, co-worker, or family member, participants reported that the stress becomes exponentially greater. A career firefighter in a rural area explained,

We're such a small community, we know everybody ... When we're sitting up here and tones drop, all of our hearts stop 'cause we're like who's that gonna be? Is that gonna be my loved one, is that gonna be somebody I hang out with everyday or work with ... It's tough sometimes, especially when you have to work on people that you work with everyday, or you know, love, and you gotta go pick them up. Makes it a little difficult to 
deal with.

Interestingly, the three focus groups that brought up the stress of knowing a victim came from departments in isolated rural, small rural, and large rural areas of the state. Therefore, fear of knowing the victim of an emergency seems to be a shared experience of each of these firefighter communities.

To cope with these various stressors, participants described a number of strategies they use for stress management. The two most commonly discussed coping strategies were departmental debriefing (12 mentions in four focus groups) and joking (nine mentions in three focus groups). Half of the focus groups described debriefing that takes place at the department after a call, providing firefighters an opportunity to vent about what they saw, what happened, and how to move forward. One participant explained that these debriefs are important because it provides an opportunity to voice an opinion, "You can voice your opinion and that let, that helps get rid of some of the stress, because we're all, we've all been there. I mean, most of us have been there." Another participant explained the importance of these debriefs before going home, as they provide an opportunity to try and relax, and transition away from work before returning to their families.

Another powerful form of coping that was talked about in three of the focus groups was joking. Laughter provides a way for firefighters to release the stress that they experience while on a call. One participant explained,

In a way you might think firemen have a sick sense of humor, you know, but we have to do it to keep ourselves sane because if we didn't joke about some of the things we saw and just you know, kept it in, you'd see a lot more firemen with a lot more psychological problems. I mean, you have to come back and you have to joke around, because if you 
don't that stress will really get to you and it will take a toll on you.

Participants later explained that the joking and poking fun is at each other, not the public, and joking and laughing are a powerful form of stress relief.

Nutrition. Nutrition was the second most frequently discussed topic within the focus groups (148 mentions). More specifically, participants spent a majority of their time speaking about barriers to eating healthier (112 mentions). Cost of healthy foods (13 mentions in six focus groups), lack of time (10 mentions in six focus groups), personal preference for unhealthy food (nine mentions in four focus groups), and disrupted meals (seven mentions in four focus groups) were the three barriers talked about most. Related more broadly to access and availability, six focus groups discussed the cost of unhealthy foods compared to healthy foods. Though many participants expressed an understanding of the importance of healthy eating, many discussed the fact that healthier food is more expensive, eliminating the choice of having healthy options around. One participant explained his frustration when shopping, "You can go down there to the store, you can get a two-liter bottle of Coke for 99 cents. You can get a 20-ounce bottle for \$1.29. Or a bottle of water's \$1.99." Another participant expressed the same frustration when eating out, “I've tried it, eating healthy is more expensive. I mean, where's Subway's dollar menu? You know, you can’t get full on $\$ 4.00$. I can’t get full on $\$ 4.00$ at Subway." Six of the eight focus groups also discussed lack of time. When considering the demands placed on firefighters between working, volunteering, firefighting, caring for family, training, and other responsibilities, firefighters feel that they simply do not have time to eat healthier. One career participant explained, “Sometimes you just don’t get time to bring food in and cook, you just have to eat when you're out, you don't have a choice. It's the only time you get a chance." Another volunteer participant echoed the same struggles, 
Always being on the go, it's not too easy to, especially volunteer wise again, you're working a full-time job, you're doing a, you know, you're not always eating the best. You know, you can't always sit down to a full, four-course meal and, you know, enjoy it. Another barrier was discussed in half of the focus groups. Firefighters explained that they simply preferred the taste of unhealthy foods. One participant said,

For me, it's just my personal habit. If I have my choice between white bread and wheat bread, I'm always gonna take the white bread ... It's like sausage or turkey burger, you know. I take the sausage. I just, that's my taste. I like the unhealthy stuff.

Because they enjoy the taste of unhealthier foods, their motivation to switch to healthier options was not strong. Finally, four of the focus groups talked about disrupted meals by telling stories about taking the time to cook a healthier meal, but after taking only one or two bites, they were interrupted by an alarm. Unfortunately, by the time they returned to the department, the meal was no longer good, or was thrown out, and unhealthy options became the replacement meal. One participant shared,

I don't know how many times I've sat down at dinner and you start to eat and pager goes off and up you're going and go and time you get back it's cold or it's thrown away ... You come back and if it's cold or you're not in the mood to eat because of whatever you went on.

After a certain time, firefighters then become trained out of trying to cook healthy meals because they are so often interrupted. One participant explained, “After so many times of being interrupted you're like, do I really want to start this, because the last two times I've done it [cooked a healthy meal], it gets interrupted." Other categories of nutrition barriers discussed in the focus groups included cooking for different tastes and the ease of unhealthy foods. 
Participants also mentioned briefly some facilitators to healthier eating such as planning their food, social support from others, and perceiving their food as fuel for firefighting.

Although numerous barriers were discussed during the focus groups, the house tours highlighted a structural facilitator, large kitchens. All of the firehouses, both career and volunteer, had a large kitchen at their disposal. The volunteer departments often had industrial kitchens because the house would be used as an event site, or large meals would be cooked for fundraising events. Only one of the career departments had an industrial sized kitchen, but the other three had full kitchens with all of the necessary appliances (stove, oven, refrigerator, microwave) to cook healthy meals.

Nutrition was also represented in the Photovoice data. A total of 27 pictures $(50.0 \%$ of pictures sent) focused on nutrition, making it the most represented of the three themes. Of those 27 pictures, $11(40.7 \%)$ showed the firefighter making a conscious healthy choice with whatever they were eating. For instance, one firefighter sent a picture of a salad and explained, "Taking time to eat a healthy lunch instead of fast food large salad and water." Another firefighter also sent a picture of his meal, and said, "Thursday lunch. This is a particularly health[y] lunch, except for the fact that the chicken salad croissant was twice what you see. I saved the other half for dinner..." Another common theme related to nutrition represented in six pictures was that of time. One firefighter took a picture of an almost empty fridge and shared, "Have not spent much time at home past few weeks...." After sending a picture of his car, another participant shared, "Yesterday was so stressful on the road from 9:30 am till 11:30 pm not much time to eat good it was McDonalds on the run." Positive motivation, conscious unhealthy choices, negative cues, and food as fuel were each represented by two pictures each. Work, availability, water, negative social pressure, fatigue, and convenience were each represented by one picture each. 
General factors impacting health. Beyond specific concerns related to nutrition, physical activity, stress, and sleep, participants also talked about generic factors unrelated to a specific aspect of health that influence their health behaviors (144 mentions). Within this category of factors, participants once again spoke three times more about barriers to their health (90 mentions) than facilitators of health (31 mentions). First, participants discussed the negative influence of barriers from their work as a firefighter, specifically mental and emotional stress (nine mentions in five focus groups). Though some participants were unable to articulate exactly how stress impacts them, all felt that stress was a barrier to their health, and many believed stress to be the number one reason for the health problems among firefighters. Another commonly discussed job barrier, mentioned in half of the focus groups, was the uncertain schedule of firefighters (six mentions). Both career and volunteer firefighters explained that they can attempt to plan physical activity or healthy cooking in their day, but because they could be called out at any given moment, they are often interrupted during their attempts at healthy living, or do not even try to be healthy. One participant shared, "We joke and say it's like war, you have to eat when you can and sleep when you can because you don't know what's going to happen when." Last, three focus groups talked about the need to find a job for everyone in the department, regardless of their health (four mentions). Because the departments are so short-staffed, departments are not able to turn people away because of health concerns. Instead, the firefighters work to find a job for any active firefighter in their department. One participant explained the experience of their chief,

The chief here told me one time that like he was hinting on you know, he may not be able to tag along, but he might be able to run an engine or a pumper or a tanker. I mean, there's a job for everybody. And, traffic detail. Anything, roll up hoses, wash hoses, I 
mean, there's lots of stuff that you know, everybody can do.

Instead of turning potential firefighters away because of potential health concerns, everyone is welcomed with open arms, and those perceived as unhealthy are simply given less stressful jobs. Other barriers to health included society, weather, age, and lack of motivation. Facilitators were also discussed on a less frequent basis, which included making healthier personal choices and the improving health of the department. It is worth noting that six of the focus groups identified heart attack as the number one cause of on-duty death for firefighters. Participants reported that their awareness of the on-the-job risk they face motivates them to lead a healthier lifestyle; however, that awareness did not always translate into taking action because of the various barriers that firefighters face.

Similar to the focus groups, participants sent pictures in the Photovoice portion of the study that also represented miscellaneous or general factors that impacted their health, shown in 15 of the 54 pictures sent $(27.8 \%)$. The most commonly represented theme was of time, without specific discussion about nutrition or physical activity. One picture was of a clock hanging on a wall, and the participant explained, "Never enough time. Just left the office, fundraising meeting at the fire house in an hour." Another theme shown more than once was of tobacco use, specifically through a picture of chew ("With it not healthy. Without, eat too much, still not healthy.") and cigarettes (“It's effects are obvious!"). Sleep was represented in the miscellaneous theme as well. One participant sent a picture of one of the trucks used at their department and said, "Early morning call lack of sleep." Similarly, the second picture was also of a department truck and explained, "No sleep, house fire last night. Up all night, work all day." Stress was the other theme represented twice. For example, one picture showed a woman's hand wearing a wedding ring, and the participant explained, "Stressful not knowing how it will go a lot of 
thinking depression thinking of the bad things." Other sub-themes represented in pictures included work, on-the-job safety, improving weather, social obligation, health warnings, convenience, environment, and stress management.

Physical activity. Similar to other factors, when discussing physical activity (117 mentions) participants discussed barriers (79 mentions) twice as often as facilitators (38 mentions). Overwhelmingly, the two most discussed barriers were weather (12 mentions in five focus groups) and lack of access to equipment (12 mentions in three focus groups). Five of the eight focus groups discussed weather, specifically winter weather, as a barrier to engaging in physical activity. One participant simply stated, "Frankly, when it gets cold, I just don't wanna move." Though many career departments have access to equipment at their department, participants still discussed a desire to be active outside, which they do not see as an option during the winter. One career participant said, "This is the biggest station we have, and you feel like a rat in a cage [in winter] because it's just, you know, you can't get out." Volunteer participants also expressed limited physical activity in the winter, as one firefighter explained,

Winter's a tough time here too, because you can't run a lot of times because of ice, if I did run. But I mean there's a lot of stuff you can't do outside because of the weather, and I think we do a lot better here in the spring, in the summer, and the fall. You know, there's like three months here where we sometimes have trouble doing anything but shoveling the driveway, and I do that with a machine.

Moreover, firefighters' preference in the winter is to be sedentary; participants mentioned a pull toward "hibernating" rather than being active once it becomes cold. Another barrier talked about in three focus groups was the lack of access to workout equipment. One volunteer explained that activity for smaller departments might be more challenging than for larger departments because 
of the lack of access to equipment. Firefighters that did not have access to equipment felt they would use it if it was made available, but for many departments that was not currently an option. Other barriers discussed were laziness, lack of requirements, lack of time, and priorities.

Conversely, although lack of access to equipment can be a barrier, having access to such equipment was the most commonly discussed facilitator to physical activity (seven mentions in two focus groups). Two focus groups discussed that having exercise equipment available at their department made engaging in physical activity that much easier. One career firefighter explained, "I mean, we're lucky here, we have pretty much weight rooms in every station. I think there's a lot of departments out there that probably doesn't have that." Another career participant in the same focus group elaborated,

I mean, us, it's pretty handy because we have them [weight rooms] at the stations. Now if we had to send our guys to the gym, to say [a local gym] there on south side, that might create big problems, you know what I mean. Might be harder.

Outside of the department, two focus groups also explained that firefighters are often provided membership discounts at local gyms. Therefore, even if the equipment is not available at the department, firefighters can use local gyms to be active.

Access to physical activity equipment was a clear theme that came from the house tours as well. All four career departments had fitness equipment, three of which had designated fitness rooms, and one had a treadmill and a few weights available in an office. Even though the paid chiefs and firefighters discussed the ease and convenience of having fitness equipment on site, two of the four career departments had the equipment located in an area away from where the firefighters normally "hang out"; therefore, while it was on site, the equipment might not be used as much as expected. The two departments with fitness rooms near common areas of the house, 
however, discussed that their fitness rooms are used quite frequently. Another important point discussed at the large rural and urban career sites was that they have multiple houses throughout one city, so while one house might have a fitness room available, firefighters serving at other houses would have to travel to use the equipment, creating an additional barrier. Despite complaints about equipment not always working, not being up-to-date, or not being sufficient for specific types of exercise, many present for the house tours liked the fact that the equipment was there, and often wanted to have more, but their budgets would not allow for it.

None of the volunteer departments had fitness equipment available at the department. One chief discussed an upcoming renovation and expansion, which included the addition of a fitness room. Another volunteer chief mentioned the limitations of the building they were in, and because of its size, housing any fitness equipment was not an option because they could barely fit the required firefighting gear. The volunteer firefighters that joined on the tour discussed interest in having equipment at the department, but understood that space and/or budget restrictions made that difficult. Additionally, they at times doubted whether or not they would actually use the equipment, even if it was made available to them.

Another unique aspect of the career departments compared to volunteer departments was a common room with a large-screen television and numerous recliners and/or couches. This was present in all four of the career departments, and at times was in front of, or along the path to, the fitness room. One group of firefighters mentioned that the chairs were not as comfortable as they looked, but the other three admitted that the recliners and television created a major temptation to engage in sedentary behavior rather than staying active.

Participants discussed physical activity in the Photovoice portion of the study as well. A total of 13 out of 54 pictures $(24.1 \%)$ related specifically to physical activity. Obligation was 
represented in two pictures, one from a career firefighter and one from a volunteer. After sending a picture of new running shoes, a career participant said, "Just went and bought these cause you made me feel bad about being fat!" Conversely, a volunteer firefighter shared a picture of a snow shovel, explaining it as "forced exercise!" Another theme represented more than once was preferred activity. One participant shared a picture of a Ping Pong table and explained, “One of my favorite ways to be active without really even noticing it." The other picture showed a snowcovered mountain, and said, "Saturday, skiing at [a local] mountain." Two firefighters also each sent a picture representing the positive influence of improving weather on their physical activity. One sent a picture of the five-day forecast available on his phone and explained, "There's nothing more motivating than signs of spring in the forecast!" The other participant sent a picture of the sun through a tree and said, "Getting outside after a long and cold winter and being more active." Other pictures related to physical activity showed sub-themes of incentive, convenience, extrinsic motives, conscious healthy choices, stress management, positive cues, sedentary behavior, negative weather, and time.

Sleep. According to the participants, being a firefighter has significant negative effects on sleep patterns (66 mentions). All eight of the focus groups talked about their sleep being consistently interrupted throughout the night (17 mentions). Even if they do not travel to a call, should an emergency call come in during their normal sleep hours, they are awakened by their alarm. One volunteer participant explained that in the past he would not go back to sleep after a call, "I've come home and then just took a shower, drank, made my coffee, and went to work. Didn't even go back to bed." Career firefighters experience similar disruptions, as one participant explained, "It's very seldom that you may get a two to three hour window of sleep without being woken up in the middle of the night." In the same way, these late night calls also 
lead to sleep deprivation or a lack of sleep, a topic discussed in six of the focus groups (10 mentions). One volunteer participant admitted that a full eight hours of sleep is not possible, saying "What they're talking about, full night's sleep, you don't get that if you work or volunteer or something like that. You know, it's just not there." Unfortunately, even when firefighters are able to sleep, two focus groups mentioned that the quality of sleep is not good.

Motivation. Participants often spoke of the importance of motivation in leading healthy or unhealthy lives (63 mentions). Even though motivation was commonly discussed in how it impacts nutrition or physical activity, participants also took time to discuss different factors that impact their motivation overall. The most commonly discussed facilitator of motivation (46 mentions) was factors related to the job of firefighting (14 mentions). Six of the eight focus groups explained that their performance while on a call could greatly increase their motivation to make healthier choices, particularly when they become easily fatigued or winded on a call. One firefighter described being motivated to make healthy changes after "a call that you go on and you use up three bottles of air and come back and you think, I've gotta do something! I am dying here!" Concern about the performance of firefighters next to them also can encourage healthy change. One department decided to implement mandatory physical activity each day because, as one firefighter explained,

Nobody wants to have to save each other, you know. You don't want four guys in shape and one guy not. We don't want to have to not count on that guy, you know. We've all got each other's backs. If I'm going in with him, I want to know he's physically able to drag me out of a fire if he needs to and vice versa. We don't wanna have to be worrying about our brothers falling down on us, you know, not being there, 'cause our lives are in each other's hands essentially. 
Time. The influence of time was mentioned in regards to a number of different behaviors related to health. However, all eight of the focus groups discussed the time demands that come with trying to balance working, firefighting, and having a family (22 mentions). Volunteer departments discussed time demands as they related to working a full-time job, then serving at the fire department in between normal work hours, while spending time with family, and many trying to raise children.

Career departments discussed the same concerns, including the time constraints related to working a full-time job as a firefighter, and also working at least one other job, if not two. One career firefighter explained, "Most of us have a second job, so as soon as we get out of here at eight o'clock, you know, they're going to another eight hour job." With all of the demands on their time between firefighting, working, and maintaining a personal life, firefighters felt it was near impossible to find time to cook healthy meals or engage in physical activity.

Potential solutions. Firefighters across the eight focus groups agreed that health and obesity are problems in the fire service; however, less agreement was reached in discussing potential solutions. One focus group even discussed the idea that there are no potential changes that could be made that would benefit the health of firefighters in West Virginia. However, other firefighters discussed three potential options: incentive programs (40 mentions in five focus groups), education (20 mentions in five focus groups), and physical requirements (13 mentions in five focus groups).

Firefighters discussed different interventions that could be created to encourage and motivate a healthier culture. First, departmental incentives were considered. One focus group said that offering equipment to departments as incentive for showing health improvements would motivate them to be healthier. Two other focus groups thought that having a budgetary incentive 
would also help; offering the department money for showing health improvements among their firefighters. Beyond the department, firefighters also talked about personal incentives. Finally, two focus groups discussed the power of a financial incentive, or offering a bonus structure to firefighters that are able to maintain a certain level of health each year.

Along with interventions and incentives, five focus groups discussed their openness to education. Free nutrition education was the most popular request, mentioned by four of the eight focus groups. As one career firefighter shared,

If they would come down here and either educate fire department personnel on how they could eat healthier, because we probably don't know all. I mean, we all think we know that eating healthy is expensive, takes more time and it's just cheaper and easier to go with fast food, but maybe they could develop a plan that would work with our schedule being as hectic as it is. The quick, healthy food.

Building awareness of the obesity and health problem within the fire service, and stress management or stress education were each discussed by two focus groups. Another opportunity for education discussed was exercise education specific to the needs of firefighters.

The last potential solution, discussed by five focus groups, was creating physical fitness requirements for all firefighters, a requirement that does not currently exist beyond initial recruitment. However, while this solution was mentioned, most firefighters wanted to discuss barriers to this approach; only two focus groups discussed this change as a viable option. One barrier to implementing a physical fitness requirement was the already limited staff at many departments. One focus group acknowledged that many of their firefighters would likely not pass a physical fitness test, and they would then be forced to find replacements, which they would not be able to afford. A participant explained, 
In a volunteer, it [mandatory fitness requirements] would drive, we would lose members and volunteer fire departments are short-staffed now. You can't afford, we can't afford to lose any more members. Anytime we lose a member, that's a guy that's not there to back you up. The fire department invests a lot of money for everybody, with the cost of gear and training, I mean it's not an option. We cannot have a program that would, unfortunately, making them be healthy 'cause we would have to get rid of people.

Similarly, one participant echoed that losing members because of mandatory fitness requirements could also lead to departments closing,

You go to these little departments out in the sticks and there's guys that are four, five hundred pounds that are firemen ... If they would go and have that physical done, would they pass? Probably not. So you take that little department and you take those couple guys that are overweight, that aren't fit, and what does that leave you with? Probably two or three guys. So you have two or three guys in a small department, what good is that gonna do you? None. It's not gonna be long and they're gonna be shut down. In addition, because of the time demands put on firefighters through their training; the requirements to become a firefighter are already so great that if further requirements were set (i.e., physical fitness), firefighters are concerned that recruiting would become even more challenging, or that some current volunteers might quit. Two focus groups also talked about negative previous experiences with fitness requirements, and the rebellion that ensued after firefighters tried to meet those standards. The previous requirements that were set were eventually eliminated because people took advantage of disability programs offered after failing fitness requirements.

Career versus volunteer. Though career and volunteer firefighters perceived significant 
differences between the two cultures, few differences came from the focus group discussions. Statistical tests showed that there were no significant differences in the amount of mentions for the various themes based on type of department (career versus volunteer) or setting (isolated rural, small rural, large rural, urban). Most common barriers to health behaviors, such as lack of time, cost of healthy foods, stress, balancing multiple jobs, lack of motivation, and disrupted sleep were echoed in all discussions. Suggestions for potential solutions were similar as well, emphasizing the importance of education and different incentives such as financial or budgetary incentives. These results show that the experiences of career and volunteer firefighters might be more similar than expected in relation to factors that influence health and wellness.

\section{Discussion}

Overall, WV firefighters identified six factors that influence their health: stress, nutrition, general factors impacting heath, physical activity, sleep, and motivation. Additionally, participants shared a number of different potential solutions for addressing the health disparities within the fire service, including incentive programs, education, and setting physical requirements.

Many of the themes that arose from the data were similar to findings from previous studies. For instance, despite firefighters' awareness of the high risk of cardiac death, participants in the current study admitted that they do not exercise regularly or eat well, a finding similar to that of Frattaroli et al. (2012). In the current study, CFFs and VFFs were quick to outline barriers that prevent them from engaging in health behaviors, such as having to balance multiple jobs and roles, limited food options, few resources, and lack of support, barriers discussed in other studies as well (e.g., Frattaroli et al., 2012). Additionally, firefighters in the current study discussed that the lack of mandatory fitness standards not only decreased 
motivation among firefighters to stay active, but also led to confusion about the best types of activity to stay fit for firefighting, which is similar to the findings of Jahnke and colleagues (2012). Disrupted sleep was another major barrier identified by participants, partially because they anticipate calls and do not want to miss a potential alarm, another concern echoed in results from Jahnke and fellow researchers (2012). Finally, participants discussed financial and budgetary concerns with regards to physical activity (e.g., cost of workout equipment, gym memberships, so forth), which mirrors concerns from both the fire service (Jahnke et al., 2012) and rural areas in general (Parks et al., 2003).

One unique barrier that emerged from the data that was not found in previous research was finding a job for everyone despite their current health. Because the departments are already so short-staffed, firefighters would rather assign less strenuous jobs to unhealthy firefighters than have to turn people away from the service. Inadvertently, however, this decision accommodates unhealthy behavior, and could reinforce sedentary and less healthy firefighters to stay engaged, potentially putting people at greater risk while at the scene of an emergency. Though one focus group did acknowledge the risk inherent in this process, other focus groups described the necessity of keeping as many active firefighters as possible.

Another interesting theme that dominated discussions about stress was the firefighters' concern about knowing the victims of an emergency to which they are called. In other health research, the closeness of a community is an asset to health, as it provides support and encouragement (Smith \& Christakis, 2008). However, firefighters in the current study painted a picture of times when tightknit communities become a liability for firefighters. First, it adds significantly more stress to an already stressful job in being responsible for saving someone you know and love. On a lighter note, the community is driven to help the firefighters in any way 
they can, and often donates food. However, community members typically donate unhealthy food, such as fast food and pastry items. Because members of the community donate the items, firefighters feel an obligation to accept and enjoy, perpetuating unhealthy eating habits. Although one might expect more concern about knowing the victim in small and isolated rural areas, in a rural state such as West Virginia, even firefighters in a large rural county discussed that source of stress along with firefighters in small and isolated rural regions. Though it is not possible, nor advisable, to change the familial nature of small communities, teaching firefighters strategies for approaching emergencies in which they know the victim could help them manage the stress better, allowing them to more efficiently perform their duties. Finally, it is possible that interventions could harness this information to create campaigns to motivate health behaviors behind the slogan such as "Don't let your neighbor down! Eat well and be active today to save a life tomorrow."

Beyond the general discussions of health that took place, a discouraging pattern also emerged, showing inconsistencies between thoughts or experiences and action. For instance, the most frequently discussed facilitator of motivation was job performance; more specifically, working at an emergency and suddenly feeling out of breath or extremely fatigued. However, based on the firefighters' comments about significant barriers, this particular facilitator did not seem to have the weight of other themes, as it had not called them to action. Another interesting barrier to emerge showing contradictory thinking regarding their health behavior was a perception of current health or ability to perform the job adequately. A few firefighters shared their perceptions that they work in a department that is "relatively" healthy, so they are not as concerned with making health behavior changes. Similarly, firefighters that perceived themselves as able to adequately perform their duties were less motivated to eat healthier or 
engage in physical activity. Staley, Weiner, and Linnan (2011) found that firefighters defined fitness as successfully being able to perform their job, regardless of other aspects of health and wellness. Therefore, because firefighters feel they are performing their duties adequately, they believe they are fit enough, and see no reason to make health behavior changes. Unfortunately, overweight and obese male firefighters have been found to underestimate their weight group (Baur, Christophi, Tsismenakis, Jahnke, \& Kales, 2012), so firefighters might be overestimating their general health and fitness as well. This concern and negative attitudes toward fitness standards might present a significant cultural barrier to health interventions designed for this population.

Within the focus groups, more inconsistencies and fatalistic thinking arose. For example, participants identified disrupted meals as a barrier to healthy eating; however, firefighters are likely interrupted more often when eating unhealthy meals. If being interrupted during a healthy meal is a barrier to healthy eating, one might expect that being interrupted during unhealthy meals should be a barrier to unhealthy eating as well. However, this logic did not emerge. Additionally, though the average BMI put many firefighters in the borderline obese category, many participants still felt that their department was "healthy." Moreover, firefighters are able to find time to go to a fast food restaurant, wait for their food, and then return to the department, but complain that they lack the time to prepare healthier options. Finally, a vast majority of the time in the focus groups, as well the majority of photos submitted, discussed barriers to health behaviors as opposed to facilitators of health. When firefighters talked about mandatory fitness requirements, their focus was also on the negative, believing that many of the firefighters in the state would not pass such requirements. The firefighters' fatalistic perspective about barriers to change and mandatory requirements shows an inherent disbelief that healthy change could be 
successfully implemented. However, facilitators of health behavior do exist in the fire service, including awareness of risk, job performance, planning and preparing, and the brotherhood of the fire service, facilitators that were represented in the focus groups and Photovoice. If these facilitators were to be successfully utilized as part of a targeted intervention, firefighters' motivation to engage in various health behaviors might increase.

\section{Potential Interventions}

Based on the house tours, some simple changes could be made within the departments to encourage health behaviors. For instance, career departments with exercise rooms could move their televisions from the common room to the exercise room, or bring exercise equipment into the common room. This small change might encourage the firefighters to move rather than sit and watch television during their downtime. Getting rid of the recliners and comfortable sofas that were in many of the common rooms might also encourage more physical activity rather than sedentary time. At departments that do not have exercise rooms, much of the firefighting equipment could be used in place of traditional exercise equipment during physical activity, particularly for strength training. Finally, rather than sitting during departmental meetings, chiefs or other firefighters in leadership positions could encourage "walk and talks," or meetings that take place while walking and moving. This strategy would allow firefighters to still be productive while engaging in physical activity.

Beyond small changes within each department, larger interventions could be organized as well, such as increased health-related education. Firefighters in multiple focus groups expressed openness to education in a variety of areas, including nutrition, exercise plans specific to the needs of firefighters, and stress management. Previous research identified firefighters' openness to education as well (e.g., Scanlon \& Ablah, 2008), and supports the idea of continued education 
for firefighters in helping decrease the risk that firefighters face on-the-job (e.g., Durand et al., 2011). In some ways, firefighters are a captive audience for nutrition programs because they have down time between calls and they are often waiting in small groups. Nutrition education focusing on quick, easy, inexpensive healthy meals could change firefighters' perceptions about the barriers to healthier eating. However, in rural areas, there is a dearth of licensed professionals to provide these sessions, so developing web-based or mobile resources might expand reach. Additionally, physical activity education directed toward the work firefighters perform could increase their motivation to participate since they could see purposeful benefits while performing their duties. Specifically, teaching firefighters about the effectiveness of even five to ten minute bouts of physical activity could change the all-or-nothing perspective that they must workout for at least an hour or it is not worth their energy. Furthermore, posting healthy prompts throughout every room in the firehouse could keep health behavior at the forefront of the firefighters' minds, encouraging change. These cues and behavioral prompts have been shown to be highly effective in motivating short-term behavior change (Community Preventative Services Task Force, 2005). Finally, stress management, including identifying other successful coping strategies and managing the stress response, might help firefighters control their level of stress. Overall, firefighters are aware of the general health concerns facing the fire service; however, they are unaware of the dangerous extent of the problem, and do not know successful strategies for improving health disparities. Successful education interventions could close that gap and help firefighters learn realistic strategies for making change.

Beyond education, a likely next step would be implementing an exercise, nutrition, or health intervention. To increase motivation to comply with such an intervention, researchers should consider providing incentives for progress in health behaviors such as fitness or healthy 
eating. Financial (giving money to individual firefighters) and budgetary (giving money to the department to supplement their budget) incentives were the most frequently discussed. One concern about this approach might be the firefighters developing dependence on extrinsic rewards for health behaviors. Even though extrinsic motivators might be less helpful for longterm behavior change, extrinsic motivation can be helpful in meeting short-term goals and initiating changes in behavior (Deci \& Ryan, 1985). Therefore, using extrinsic incentives at the beginning might jump start firefighter's motivation, at which point more intrinsic motivators can be identified to continue the behaviors long-term.

Though debate exists among firefighters about whether fitness requirements should be developed (e.g., Frattaroli et al., 2012), requirements would decrease the risk faced by firefighters on the job by ensuring that minimum levels of health are met and maintained, while facilitating motivation for health behavior. Requirements should, however, encourage health by offering benefits to firefighters who meet or exceed the standards consistently. Also, firefighters who fail to meet requirements should be given a probationary period to meet guidelines, while given access to educational resources to teach them how to incorporate health behavior into their daily lives.

It is important to note that, based on the current findings, any intervention that is developed for firefighters should address stress and sleep, along with nutrition and exercise. Many interventions in the past have focused solely on developing physical activity programs (e.g., Roberts, O'Dea, Boyce, \& Mannix, 2002) or nutrition education (e.g., Frattaroli et al., 2012). However, stress was the most frequently discussed theme in the focus groups, and disrupted sleep, another commonly discussed problem by the firefighters, has been associated with weight gain (Cappuccio et al., 2008) and greater risk of death (Cappuccio, et al., 2010). A 
complete intervention would address health from all angles, including physical activity, nutrition, stress, and sleep. Furthermore, interventions should attempt to address factors at multiple levels of SEM. Results show that factors at every level of SEM interact to influence the health of firefighters (see Tables 5 and 6, and Figures 1 and 2). Without addressing multiple levels, interventions will be limited in their effectiveness because they will only be focusing on a small portion of the larger, complex picture.

\section{Recommendations for Future Research}

As mentioned previously, the problem of health disparities within the fire service is a complex problem requiring comprehensive interventions and approaches (Poston et al., 2011). Throughout the focus groups, firefighters expressed fatalistic thinking with regards to health. Rather than being optimistic that health disparities would be solved, firefighters were quick to identify myriad barriers that prevent healthy living, explaining that overcoming those barriers proves to be incredibly challenging in the fire service; some even expressed disbelief that any change could help firefighters become healthier. Before researchers attempt any sort of intervention, identifying and changing such fatalistic beliefs is pivotal. One way to begin that process might be an in depth ethnographic experience, to learn more about the source of such fatalistic beliefs, and possible strategies for reversing said beliefs.

Once their fatalism has been addressed, researchers should begin examining the efficacy of various educational programs, including those addressing physical activity, nutrition, and stress management. Though a number of interventions have been done in the past, few have been effective in significantly improving the health of firefighters (Staley et al., 2011). Therefore, research should continue exploring a variety of education and incentive programs to find which would be most helpful. Utilizing Photovoice methodologies could help researchers develop more 
effective interventions by providing in depth understanding of the firefighter's experience outside of the firehouse. To reach a larger population of firefighters, researchers might also consider developing web-based resources and studying the effectiveness of such resources. Providing web-based health resources has shown some success in cardiovascular disease prevention when used as part of the West Virginia WISEWOMAN study (Carey, Cooper, Miller, Seabury, \& Staudacher, 2013), and studying the effectiveness of such resources among other populations could prove beneficial. Finally, future research should examine the potential consequences of mandatory fitness requirements. For instance, do such standards lead to dropout? Could standards serve to increase motivation if implemented correctly, and if used in tandem with education? Firefighters perceive that fitness standards would cause more harm than good; however, researchers should evaluate if this concern is valid or if simply based in a fear of not meeting the standards.

\section{Strengths}

Perhaps the greatest strength of the current study was utilizing multiple sources of data, including focus groups to capture the experience of many, Photovoice to represent individual experiences that might have been missed in a group setting, and house tours to provide a brief ethnographic experience and understanding of the settings within which the firefighters work. Previous research tended to rely on one source of data; by using multiple sources the research team was able to compare and contrast the different sources of data, creating the best possible representation of firefighters' experiences.

In addition, recruiting participants from both career and volunteer departments from various settings (urban, large rural, small rural, and isolated rural) provided an opportunity to learn about the experiences of firefighters in a variety of environments. Previous studies often 
focused on only career or volunteer firefighters. Although the participants in the current study assumed significant differences between the two cultures, results show that few differences exist in the factors that impact health behavior; differences exist mainly in the structure of the context, such as the extra time spent at the department. Additionally, much of the previous research has recruited firefighters in more metropolitan or urban areas, which likely impacts their experience with, and perceptions of, health and wellness, particularly in their access to healthy foods and opportunities for physical activity. Because volunteers represent a vast majority of firefighters throughout the country, and many firefighters serve in rural areas, hearing the voices of firefighters in various settings is pivotal to learn more about how to effectively approach the problem of health and wellness in the fire service.

Finally, the methods used in data analysis add to the trustworthiness and validity of the data. One benefit of using CQR is that the three-person research team works independently and then discusses their interpretation until consensus is met. This process minimizes the potential influence of individual bias during analysis because individuals develop their own interpretation before meeting as a group. Member checking was also used to ensure the analysis accurately represented the experience of the participants. To minimize the influence of the lead researcher's bias, bracketing was used, and the journal was shared with the three-person research team to keep awareness of potential biases high. Overall, these processes strengthen the trustworthiness of the analysis and results.

\section{Limitations}

One of the limitations of the current study came in recruitment. Volunteer departments in rural areas were difficult to contact because they often did not have e-mail, and if a phone number for the department was provided, there are often times when there are no volunteers at 
the department. Though saturation was reached among the focus groups, a larger sample size of rural participants might have shifted the emphasis of the results.

For the Photovoice portion of the study, all but one firefighter that volunteered to participate had a smartphone with camera and SMS capabilities (the exception used a tablet with e-mail capabilities to send photos and explanations). The Photovoice methodology might have attracted only those firefighters who felt technologically savvy, which could have skewed the images taken and sent. Also, the VFFs were more likely to participate in this portion of the study and therefore the themes of the photos might over represent the experience of VFFs compared to CFFs.

Finally, the timing of the focus groups might have caused weather related themes to emerge compared to other times of year. The winter this year in West Virginia was one of the harshest in recent history, and conducting focus groups toward the end of winter meant participants might have been more focused on weather than other concerns. Should focus groups had taken place in summer or fall, different barriers might have been discussed. However, it is important to know that weather can significantly influence health, at least during winter.

\section{Recommendations for the WV State Firemen's Association}

Participants in the current study expressed the importance of the support of leadership within the fire service when it comes to health behavior, a message echoed in the previous literature (Frattaroli et al., 2012), including the messages received from officers and management (Staley et al., 2011). Therefore, the West Virginia State Firemen's Association (WVSFA) has a unique opportunity to assist departments in developing a healthier environment. Some recommendations for the WVSFA include the following:

- Consult with dietitians, exercise physiologists, and other professionals to develop 
statewide recommendations for departments. Even if national requirements do not exist, departments could benefit from knowing specific health recommendations for firefighters (e.g., aerobic capacity, strength, weight, etc.).

- Resources should be created for departments with basic educational information regarding quick and inexpensive healthy eating, useful physical activity for firefighters, and strategies for stress management. Then, should departments not meet the recommendations developed by the WVSFA, they can begin implementing changes to become healthier.

- Assist departments in communicating different programs that have shown to be effective and enjoyable. For instance, one department found that a physical activity requirement of 60-minutes daily was effective in promoting physical activity among their firefighters. Similar programs could be shared with other departments around the state to share ideas of programs that are working.

- Develop an online resource for the public to donate money or healthy items to the departments throughout the state. For instance, people could donate exercise equipment, local farmers could donate fresh produce, or helping professionals could donate their time by visiting departments and providing educational classes. Having a resource for the community might encourage healthier donations so both the public and the fire service would benefit.

The WVSFA should take advantage of its unique ability to communicate and organize information from departments throughout the state to help promote healthy change within the fire service. 


\section{References}

Baur, D. M., Christophi, C. A., Tsismenakis, A. J., Jahnke, S. A., \& Kales, S. N. (2012). Weightperception in male career firefighters and its association with cardiovascular risk factors. BMC Public Health, 12(480), 1-8.

Bronfenbrenner, U. (1977). Toward an experimental ecology of human development. American Psychologist, 32, 513-531. http://dx.doi.org/10.1037//0003-066X.32.7.513

Byczek, L., Walton, S. M., Conrad, K. M., Reichelt, P. A., \& Samo, D. G. (2004).

Cardiovascular risks in firefighers: Implications for occupational health nurse practice. American Association of Occupational Health Nurses, 52(2), 66-76.

Cappuccio, F. P., D’Elia, L., Strazzullo, P., \& Miller, M. A. (2010). Sleep duration and all-cause mortality: A systematic review and meta-analysis of prospective studies. SLEEP, 33(5), $585-592$.

Cappuccio, F. P., Taggart, F. M., Kandala, N., Currie, A., Peile, E., Stranges, S., \& Miller, M. A. (2008). Meta-analysis of short sleep duration and obesity in children and adults. SLEEP, 31(5), 619-626. http://dx.doi.org/10.14341/2071-8713-5117

Carey, S., Cooper, C., Miller, B., Seabury, R., \& Staudacher, A. (2013, August). West Virginia WISEWOMAN Program Annual Report 2011-2012. Retrieved from http://www.wvdhhr.org/wvwisewoman/pdf_forms/WW_Annual_Report_2012.pdf

Chrzanowska, J. (2002). Interviewing groups and individuals in qualitative market research. Thousand Oaks, CA: Sage.

Community Preventive Services Task Force. (2005, June). Environmental and policy approaches to physical activity: point-of-decision prompts to encourage use of stairs. Guide to Community Preventative Services. Retrieved from: 
www.thecommunityguide.org/pa/environmental-policy/podp.html.

Creswell, J. W. (2007). Qualitative Inquiry and Research Design: Choosing among Five Traditions ( $2^{\text {nd }}$ ed.). Thousand Oaks, CA: Sage.

Deci, E.L. \& Ryan, R.M. (1985). Intrinsic motivation and self-determination in human behavior. New York: Plenum.

Durand, G., Tsismenakis, A. J., Jahnke, S. A., Baur, D. M., Christophi, C. A., \& Kales, S. N. (2011). Firefighters' physical activity: Relation to fitness and cardiovascular disease risk. Medicine \& Science in Sports \& Exercise, 43(9), 1752-1759. http://dx.doi.org/10.1249/MSS.0b013e318215cf25

Evans, J., \& Jones, P. The walking interview: Methodology, mobility, and place. Applied Geography, 31, 849-858. http://dx.doi.org/10.1016/j.apgeog.2010.09.005

Fahy, R. F., LeBlanc, P. R., \& Molis, J. L. (2012). Firefigher fatalities in the United States 2011. Fire Analysis and Research Division. Quincy, MA: National Fire Protection Association. Retrieved January 2013, from http://www.nfpa.org

Frattaroli, S., Pollack, K. M., Bailey, M., Schafer, H., Cheskin, L. J., \& Holtgrave, D. R. (2012). Working inside the firehouse: Developing a participant-driven intervention to enhance health-promoting behaviors. Health Promotion Practice, 22, 1-8. http://dx.doi.org/10.1177/1524839912461150

Golden, S. D., \& Earp, J. A. L. (2012). Social ecological approaches to individuals and their contexts: Twenty years of health education and behavior health promotion interventions. Health Education \& Behavior, 39(3), 364-372.

Guidotti, T. L. (1992). Human factors in firefighting: Ergonomic-, cardiopulmonary-, and psychogenic stress-related issues. International Archives of Occupational and 
Environmental Health, 64, 1-12. http://dx.doi.org/10.1007/BF00625945

Hatch, J. A. (2002). Doing Qualitative Research in Education Settings. Albany, NY: State University of New York.

Hill, C. E. (2012). Consenual Qualitative Research. Washington, DC: American Psychological Association.

Jahnke, S. A., Poston, W. S., Jitnarin, N., \& Haddock, K. (2012). Health concerns of the U.S. Fire Service: Perspectives from the firehouse. American Journal of Health Promotion, 27(2), 111-118. http://dx.doi.org/10.4278/ajhp.110311-QUAL-109

Janis, I. (1972). Victims of groupthink: A psychological study of foreign-policy decisions and fiascoes. Boston, MA: Houghton Mifflin.

Kales, S. N., Aldrich, J. M., Polyhronopoulos, G. N., Leitao, E. O., Artzerounian, D., Gassert, T. H., . . Christiani, D. C. (1999). Correlates of fitness for duty in hazardous materials firefighters. American Journal of Industrial Medicine, 36, 618-629. http://dx.doi.org/10.1002/(SICI)1097-0274(199912)36:6<618::AID-AJIM4>3.0.CO;2-7

Kales, S. N., Soteriades, E. S., Christophi, C. A., \& Christiani, D. C. (2007). Emergency duties and deaths from heart disease among firefighters in the United States. The New England Journal of Medicine, 356(12), 1207-1215. http://dx.doi.org/10.1056/NEJMoa060357

Kvale, S., \& Brinkmann, S. (2009). Interviews: Learning the Craft of Qualitative Research Interviewing ( $2^{\text {nd }}$ ed.). Thousand Oaks, CA: Sage.

Marshall, C., \& Rossman, G. B. (2006). Designing Qualitative Research (4 ${ }^{\text {th }}$ ed.). Thousand Oaks, CA: Sage.

National Fire Protection Agency (NFPA). (2013, February 15). Retrieved from http://www.nfpa.org/index.asp?cookie_test=1. 
Parks, S. E., Housemann, R. A., \& Brownson, R. C. (2003). Different correlates of physical activity in urban and rural adults of various socioeconomic backgrounds in the United States. Journal of Epidemiological Community Health, 57, 29-35.

Patton, M. Q. (1990). Qualitative evaluation and research methods. ( $2^{\text {nd }}$ ed.). Newbury Park, CA: Sage.

Poston, W. S., Haddock, K., Jahnke, S. A., Jitnarin, N., Tuley, B. C., \& Kales, S. N. (2011). The prevalence of overweight, obesity, and substandard fitness in a population-based firefighter cohort. Journal of Occupational and Environmental Medicine, 53(3), 266-273. http://dx.doi.org/10.1097/JOM.0b013e31820af362

Rhea, M. R., Alvar, B. A., \& Gray, R. (2004). Physical fitness and job performance of firefighters. Journal of Strength and Conditioning Research, 18(2), 348-352. http://dx.doi.org/10.1519/R-12812.1

Roberts, M. A., O'Dea, J., Boyce, A., \& Mannix, E. T. (2002). Fitness levels of firefighter recruits before and after a supervised exercise training program. Journal of Strength and Conditioning Research, 16(2), 271-277. http://dx.doi.org/10.1519/00124278-20020500000016

Rural Health Research Center. (2013, October 15). Retreived from http://depts.washington.edu/uwruca/index.php.

Sallis, J. F., Owen, N., \& Fisher, E. B. (2008). Ecological models of health behavior. In K. Glanz, B. K. Rimer, \& K. Viswanath (Eds.), Health behavior and health education: Theory, research, and practice ( $4^{\text {th }}$ ed.) (pp. 465-486). San Francisco, CA: Jossey-Bass. Scanlon, P., \& Ablah, E. (2008). Self-reported cardiac risks and interest in risk modification among firefighters: A survey-based study. Journal of American Osteopathic Association, 
108(12), 694-698.

Smith, K. P., \& Christakis, N. A. (2008). Social networks and health. Annual Review of Sociology, 34, 405-429.

Staley, J. A., Weiner, B., \& Linnan, L. (2011). Firefighter fitness, coronary heart disease, and sudden cardiac death risk. American Journal of Health Behavior, 35(5), 603-617. http://dx.doi.org/10.5993/AJHB.35.5.9

Stokols, D. (1992). Establishing and maintaining healthy environments: Toward a social ecology of health promotion. American Psychologist, 47(1), 6-22.

Stokols, D. (1996). Translating social ecological theory into guidelines for community health promotion. American Journal of Health Promotion, 10(4), 282-298.

Tremblay, M. S., Colley, R. C., Saunders, T. J., Healy, G. N., \& Owen, N. (2010). Physiological and health implications of a sedentary lifestyle. Applied Physiology, Nutrition, and Metabolism, 35, 725-740. http://dx.doi.org/10.1139/H10-079

Tufford, L., \& Newman, P. (2010). Bracketing in qualitative research. Qualitative Social Work, 11(1), 80-96. http://dx.doi.org/10.1177/1473325010368316

Wang, C. C. (1999). Photovoice: A participatory action research strategy applied to women's health. Journal of Women's Health, 8(2), 185-192. http://dx.doi.org/10.1089/jwh.1999.8.185

Wang, C. C., \& Redwood-Jones, Y. A. (2001). Photovoice ethics: Perspectives from Flint Photovoice. Health Education \& Behavior, 28(5), 560-572. http://dx.doi.org/10.1177/109019810102800504

Williford, H. N., Duey, W. J., Olson, M. S., Howard, R., \& Wang, N. (1999). Relationship between fire fighting suppression tasks and physical fitness. Ergonomics, 42(9), 1179- 
1186. http://dx.doi.org/10.1080/001401399185063 
Table 1

Participant Demographic Information, Means and SD

\begin{tabular}{cccc}
\hline Variable & $\begin{array}{c}\text { CFFs } \\
(\mathrm{n}=27)\end{array}$ & $\begin{array}{c}\text { VFFs } \\
(\mathrm{n}=31)\end{array}$ & $\begin{array}{c}\text { Total } \\
(\mathrm{n}=58)\end{array}$ \\
\hline Age & $36.41(8.29)$ & $40.87(14.30)$ & $38.79(12.00)$ \\
BMI & $27.99(3.85)$ & $29.47(5.22)$ & $28.78(4.66)$ \\
Total Years as a & $15.19(8.14)$ & $16.68(12.22)$ & $15.98(10.46)$ \\
Firefighter & & & \\
Total Years as a FF in & $13.44(7.55)$ & $16.55(12.36)$ & $15.10(10.43)$ \\
WV & & & \\
Total Years as a FF at & $12.30(7.20)$ & $15.61(12.13)$ & $14.07(10.19)$ \\
Current Dept. & & & \\
\end{tabular}


Table 2

Participant Demographic Information, Frequencies

\begin{tabular}{ccccc}
\hline Variable & Level & $\begin{array}{c}\text { CFFs } \\
(\mathrm{n}=27)\end{array}$ & $\begin{array}{c}\text { VFFs } \\
(\mathrm{n}=31)\end{array}$ & $\begin{array}{c}\text { Total } \\
(\mathrm{n}=58)\end{array}$ \\
\hline Income & Less than $\$ 25,000$ & 0 & 3 & 3 \\
& $\$ 25,000$ to 34,999 & 3 & 5 & 8 \\
& $\$ 35,000$ to 49,999 & 6 & 9 & 15 \\
& $\$ 50,000$ to 74,999 & 3 & 7 & 10 \\
& $\$ 75,000$ to 99,999 & 5 & 2 & 7 \\
& $\$ 100,000+$ & 8 & 3 & 11 \\
Education & Some High School & 0 & 3 & 3 \\
& High School Graduate & 5 & 16 & 21 \\
& Some College or & 19 & 6 & 25 \\
& Technical School & & & 7 \\
& College Graduate & 2 & 5 & 2 \\
& Master's or Beyond & 1 & 1 & \\
\hline
\end{tabular}


Table 3

Focus Group Frequency of Themes and Sub-Themes

\begin{tabular}{|c|c|c|c|c|}
\hline Theme & Sub-Theme & $\begin{array}{c}\text { CFFs } \\
(\mathrm{n}=27)\end{array}$ & $\begin{array}{c}\text { VFFs } \\
(\mathrm{n}=31)\end{array}$ & $\begin{array}{c}\text { Total } \\
(\mathrm{n}=58)\end{array}$ \\
\hline \multirow[t]{4}{*}{ Stress } & & 92 & 57 & 149 \\
\hline & Sources of stress & 50 & 31 & 81 \\
\hline & Coping strategies & 40 & 24 & 64 \\
\hline & Effects of stress & 2 & 2 & 4 \\
\hline \multirow[t]{4}{*}{ Nutrition } & & 81 & 67 & 148 \\
\hline & Barriers & 53 & 59 & 112 \\
\hline & Facilitators & 19 & 2 & 21 \\
\hline & General influences & 9 & 6 & 15 \\
\hline \multirow[t]{5}{*}{ General } & & 73 & 71 & 144 \\
\hline & & & & \\
\hline & Barriers & 32 & 58 & 90 \\
\hline & Facilitators & 22 & 9 & 31 \\
\hline & General factors & 19 & 4 & 23 \\
\hline \multirow{4}{*}{$\begin{array}{l}\text { Physical } \\
\text { Activity }\end{array}$} & & 55 & 62 & 117 \\
\hline & & & & \\
\hline & Barriers & 32 & 47 & 79 \\
\hline & Facilitators & 23 & 15 & 38 \\
\hline \multirow[t]{3}{*}{ Sleep } & & 34 & 32 & 66 \\
\hline & Effects of firefighting & 17 & 15 & 32 \\
\hline & Barriers & 10 & 9 & 19 \\
\hline
\end{tabular}




$\begin{array}{llll}\text { Effects of sleep habits } & 5 & 4 & 9 \\ \text { Coping strategies } & 2 & 4 & 6\end{array}$

Motivation

Facilitators

$$
\text { Barriers }
$$

Potential

Solutions
25

14

17

2

5
25

17

12

46

49

34

83

Education

Changes/Interventions

Physical requirements

Barriers to physical requirements

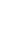


Table 4

Photovoice Frequency of Themes and Sub-Themes

\begin{tabular}{|c|c|c|c|c|}
\hline Theme & Sub-Theme & $\begin{array}{c}\text { CFFs } \\
(\mathrm{n}=11)\end{array}$ & $\begin{array}{c}\text { VFFs } \\
(\mathrm{n}=43)\end{array}$ & $\begin{array}{c}\text { Total } \\
(\mathrm{n}=54)\end{array}$ \\
\hline \multirow[t]{13}{*}{ Food } & & $4(36.3 \%)$ & $23(53.5 \%)$ & $27(50.0 \%)$ \\
\hline & Work & & 1 & 1 \\
\hline & Time & & 6 & 6 \\
\hline & Availability & 1 & & 1 \\
\hline & Water & 1 & & 1 \\
\hline & Positive Motivation & 2 & & 2 \\
\hline & Conscious Healthy Choice & 1 & 10 & 11 \\
\hline & Conscious Unhealthy Choice & 1 & 1 & 2 \\
\hline & Negative Social Pressure & 1 & & 1 \\
\hline & Negative Cues & & 2 & 2 \\
\hline & Fatigue & & 1 & 1 \\
\hline & Convenience & & 1 & 1 \\
\hline & Food as Fuel & & 2 & 2 \\
\hline Physical & & $5(45.5 \%)$ & $8(18.6 \%)$ & $13(24.1 \%)$ \\
\hline \multicolumn{5}{|l|}{ Activity } \\
\hline & Obligation & 1 & 1 & 2 \\
\hline & Incentive & 1 & & 1 \\
\hline & Preferred Activity & 1 & 1 & 2 \\
\hline & Convenience & 1 & & 1 \\
\hline & Improving Weather & 1 & 1 & 2 \\
\hline
\end{tabular}


Extrinsic Motives 1

Conscious Healthy Choice $\quad 1 \quad 1$

Stress Management 11

$\begin{array}{lll}\text { Positive Cues } & 1 & 1\end{array}$

Negative Cues $\quad 2 \quad 2$

Sedentary Behavior $\quad 1 \quad 1$

Negative Weather $\quad 1 \quad 1$

Time 11

$\begin{array}{llll}\text { Other } & 2(18.2 \%) & 13(30.2 \%) & 15(27.8 \%)\end{array}$

Time $\quad 4 \quad 4$

$\begin{array}{lll}\text { Work } & 1 & 1\end{array}$

On-The Job Safety $\quad 1 \quad 1$

Improving Weather $\quad 1 \quad 1$

$\begin{array}{llll}\text { Tobacco } & 1 & 1 & 2\end{array}$

$\begin{array}{lll}\text { Social Obligation } & 1 & 1\end{array}$

Health Warning $\quad 1 \quad 1$

$\begin{array}{lll}\text { Sleep } & 2 & 2\end{array}$

$\begin{array}{lll}\text { Stress } & 2 & 2\end{array}$

$\begin{array}{lll}\text { Convenience } & 1 & 1\end{array}$

$\begin{array}{lll}\text { Environment } & 1 & 1\end{array}$

$\begin{array}{lll}\text { Stress Management } & 1 & 1\end{array}$ 
Table 5

Results in SEM Landscape, Volunteer

\begin{tabular}{|c|c|c|c|}
\hline SEM Level & Result & Source & Illustrative Quote/Explanation \\
\hline \multirow[t]{5}{*}{ Individual } & Personal & Photovoice & "With it, not healthy; without it, eat too \\
\hline & Habits & & much, still not healthy." \\
\hline & & & -Urban \\
\hline & Personal & Focus & "Well, if we're honest, we prefer to eat \\
\hline & Preference & Groups & those [unhealthy] foods." - Isolated rural \\
\hline \multirow[t]{4}{*}{ Microsystem } & Department & House & Industrial kitchen; no exercise \\
\hline & & Tours & equipment; large, open event space \\
\hline & Family & Photovoice & “Great Gram's $100^{\text {th }}$ birthday. Not too \\
\hline & & & healthy." -Urban \\
\hline \multirow[t]{6}{*}{ Mesosystem } & Work/Life/ & Focus & "I've seen our firemen being working a \\
\hline & Volunteer & Groups & structure fire $\ldots$ and then going to work \\
\hline & Balance & & at 6 o'clock in the morning." - Large rural \\
\hline & Work/Life/ & Photovoice & "No sleep house fire last night up all \\
\hline & Volunteer & & night work all day" -Small rural \\
\hline & Balance & & \\
\hline \multirow[t]{5}{*}{ Exosystem } & Access & Focus & “There's no, we don't have an exercise or \\
\hline & & Groups & a gym or anything locally in this \\
\hline & & & particular area." -Isolated rural \\
\hline & Knowing the & Focus & "Especially if it's a family member \\
\hline & Victim & Groups & [victim of a call]. Everybody living close, \\
\hline
\end{tabular}




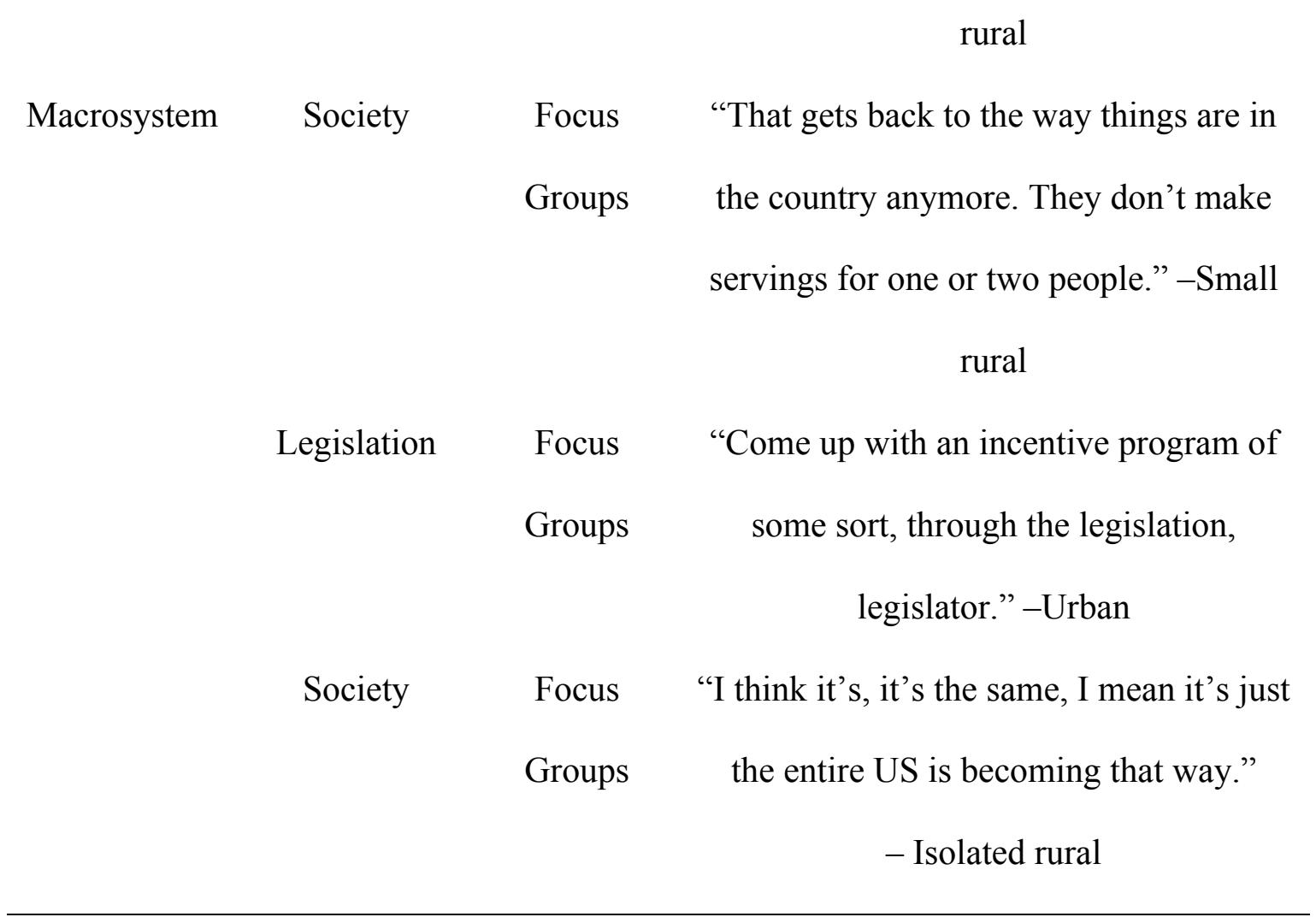


Table 6

Results in SEM Landscape, Career

\begin{tabular}{|c|c|c|c|}
\hline SEM Level & Result & Source & Illustrative Quote \\
\hline \multirow[t]{6}{*}{ Individual } & Personal & Focus & "I need to quit my cigarette habit's what \\
\hline & Habits & Groups & I need to do."-Isolated rural \\
\hline & Personal & Photovoice & "This [McDonald's sweet tea] has way \\
\hline & Preference & & too much sugar but I LOVE THEM! I \\
\hline & & & average 2 a day, 3 days a week." -Large \\
\hline & & & rural \\
\hline \multirow[t]{6}{*}{ Microsystem } & Department & House & Access to exercise equipment, kitchens \\
\hline & & Tours & with appliances, common rooms with \\
\hline & & & recliners and TVs \\
\hline & Food & Focus & "It's easy to find something [to cook] \\
\hline & Preferences & Groups & that everybody likes, but it's not gonna \\
\hline & & & be healthy." -Large rural \\
\hline \multirow[t]{7}{*}{ Mesosystem } & Work/Life/ & Focus & "I mean, I work two jobs, I work here \\
\hline & Firefighting & Groups & and the other job so on my days off ... \\
\hline & Balance & & there's a lot of times we end up working \\
\hline & & & til really late." -Isolated rural \\
\hline & Work/Life/ & Focus & "... Everybody in here works a second \\
\hline & Firefighting & Groups & job of some sort." -Urban \\
\hline & Balance & & \\
\hline \multirow[t]{2}{*}{ Exosystem } & Community & Photovoice & "Thought it was a nice gesture, but with \\
\hline & Donation & & $4 \mathrm{~g}$ trans fat per 17 chips, it's not exactly \\
\hline
\end{tabular}


promoting healthy eating. And gave us a whole case of them [chips]." -Urban Knowing the $\quad$ Focus 'It's just every fast food restaurant's Victim Groups everywhere, I mean you pass them to go anywhere pretty much in any town." -

Small rural

Lack of $\quad$ Focus "It's actually not even encouraged from Support Groups the higher ups. You would think that a company would want to promote physical fitness and activity and whatnot..."-Large rural Macrosystem Lack of Focus 'Nobody's making me do it. I'm gonna Requirements Groups make the same amount of money whether I sit here and do this [makes a channel changing motion with his hand] or if I go over there and workout." -

Urban

Society

Focus "I don't think it's just a fire service Groups thing, I think it's a society thing too. I mean you look at society, society's built around fast food now..."-Small rural 
Figure 1.

SEM Landscape - Volunteer Photos
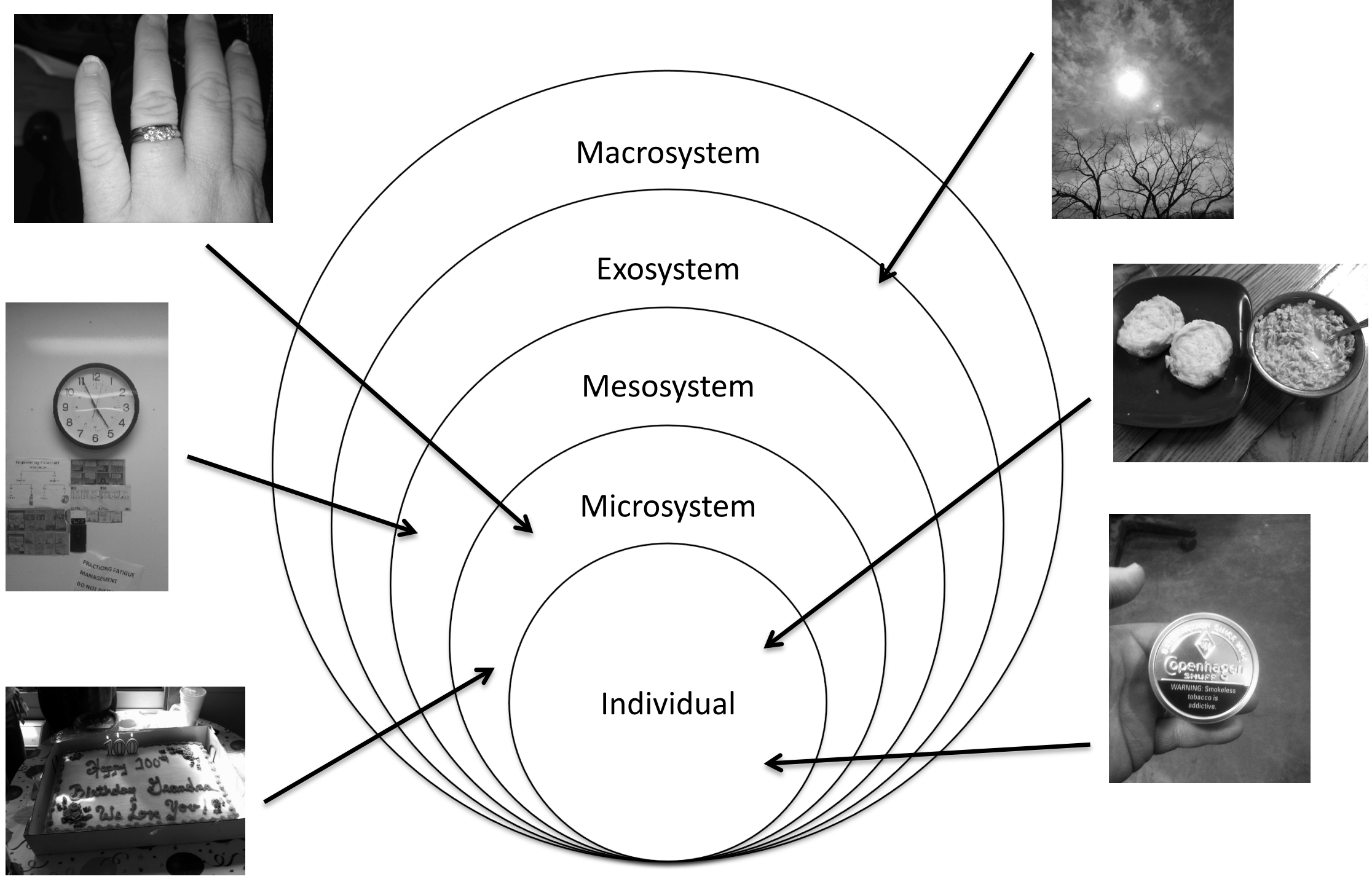


\section{Figure 2.}

\section{SEM Landscape-Career Photos}
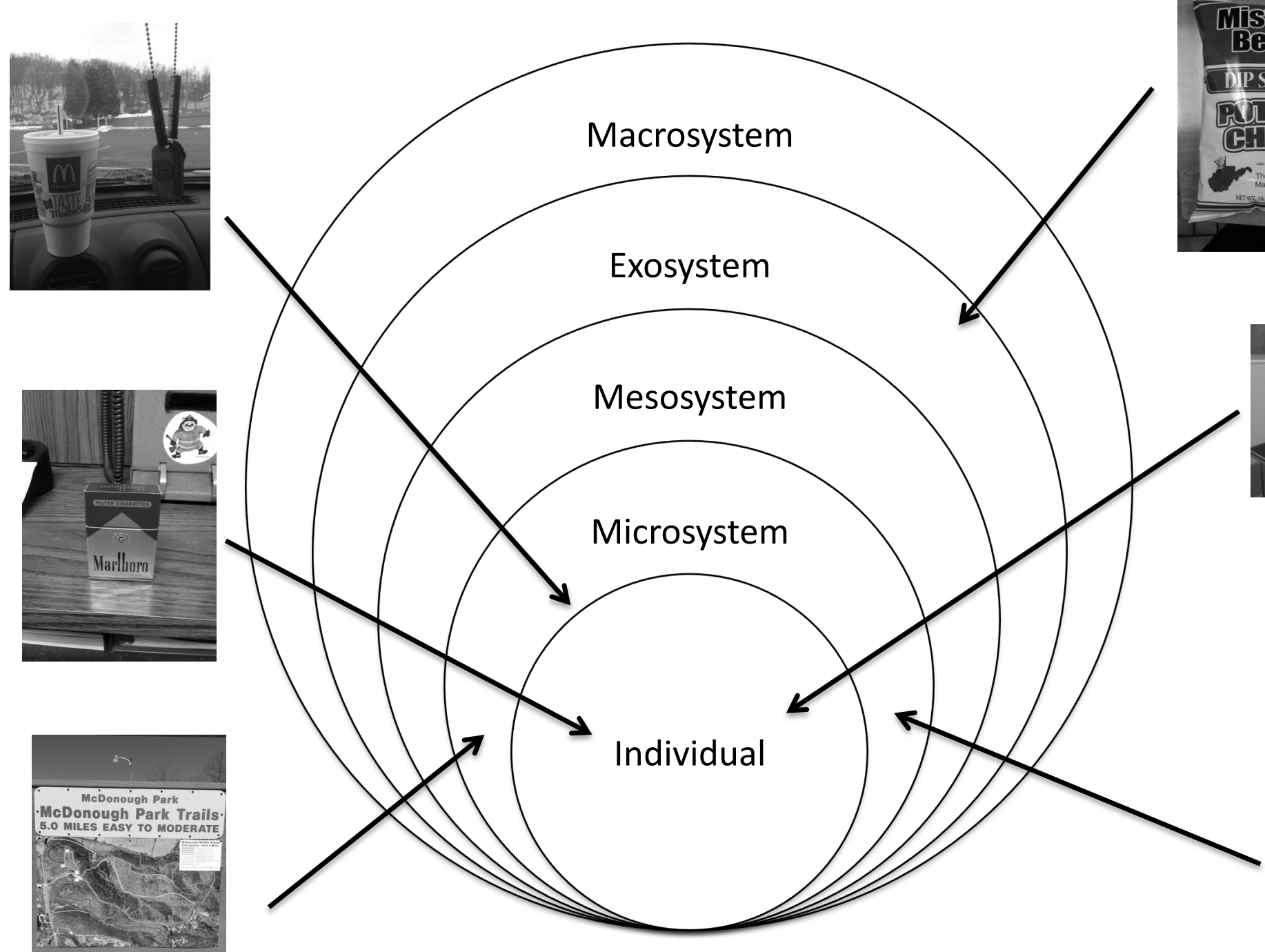

Minter

$B B^{2}{ }^{2}$

DPSTYET:-

(1.i.

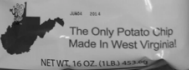
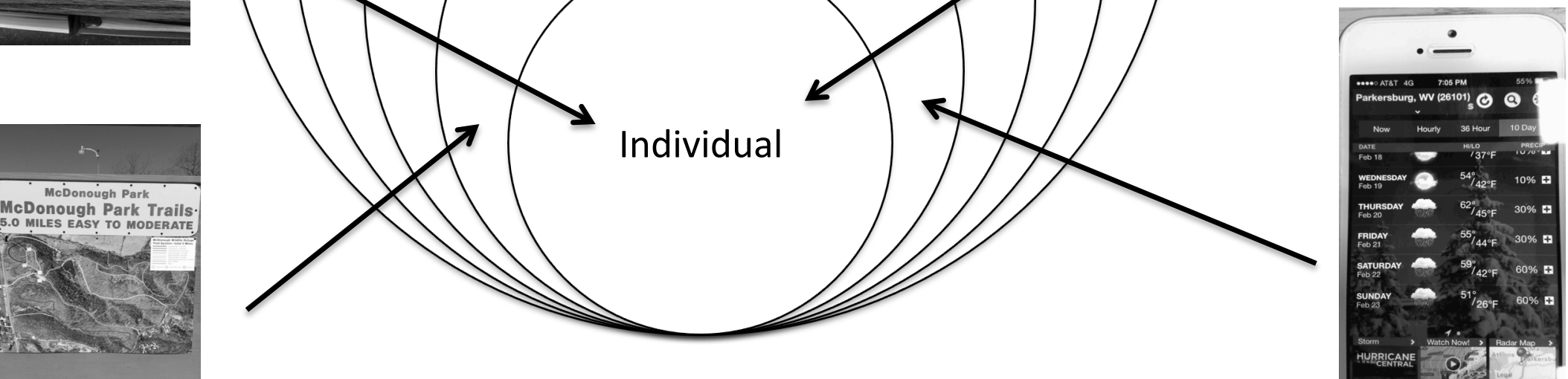


\section{Appendix A}

\section{Review of the Literature}

The obesity epidemic in the United States continues to worsen, as rates of obesity have consistently risen since 1990 (CDC, 2012). While the health risks related to obesity among the larger population are dire, even greater risk exists amongst those in helper professions, such as first responders. Because those in life saving professions are responsible for not only their safety, but the safety of others as well, an inability to effectively perform their duties could put multiple people's lives at risk. Anecdotally, many believe that first responders are in top shape; however, the escalation of obesity has affected the first responder population as well. For example, researchers have examined the culture of firefighting over the last several decades (e.g., Guidotti, 1992), and how the trends in obesity have affected a group of people trying to keep others safe. Ideally, firefighters would maintain a level of health and wellness that allows them to fulfill their on-duty responsibilities effectively. Unfortunately, rates of obesity in firefighters are as high as, or higher than, the general population (Poston, Jitnarin, Haddock, Jahnke, \& Tuley, 2011; Soteriades et al., 2005). In addition, coronary heart disease (CHD) continues to be the number one cause of on-duty deaths (CDC, 2006; Fahy, LeBlanc, \& Molis, 2012). Even though firefighters report an interest in exercise and wellness programs (Scanlon \& Ablah, 2008), interventions to help improve levels of health in firefighters have not been effective (Staley, Weiner, \& Linnan, 2011). By learning more about facilitators of, and barriers to, health behaviors among firefighters, more effective interventions can be designed (Jahnke, Poston, Jitnarin, \& Haddock, 2012b; Staley, Weiner, \& Linnan, 2011), leading to better safety and performance on the job. Without this understanding, patterns of obesity and CHD may continue to worsen. This literature review is organized into three sections: (a) the culture of firefighting 
and how that relates to perceptions of health and wellness, (b) the culture of Appalachia and perceptions of health and wellness in this specific area of the United States, and (c) the Social Ecological Model, a useful framework for examining different factors that can effect an individual's health and wellness behavior.

\section{Firefighter Culture}

Demography. According to the National Fire Protection Agency (NFPA), in 2011 there were approximately 1.1 million firefighters in the United States working at over 30,000 fire departments. Sixty-nine percent of total firefighters are volunteer firefighters (VFF), and slightly over $30 \%$ of firefighters work as career firefighters (CFF). While most CFF work occurs in areas with a population over 25,000 people, $94 \%$ of VFFs can be found in smaller communities with populations less than 25,000 people. In fact, a majority of VFFs work in areas with populations less than 2,500. Firefighters range in age from as young as 16 , to over 60 years old, with the vast majority (75\%) falling between 20 and 49 years old. The number one reason for firefighter response in the past couple of years was medical assistance, accounting for two out of every three calls, followed by false alarms. In total, firefighters across the country responded to over 26.5 million calls, but only $5 \%$ were due to fire. As of 2010 , CFFs tend to be mostly males; only $3.6 \%$ of CFFs are women. Similarly, CFFs are predominantly white; research reports approximately 7\% African-American and 10\% Hispanic firefighters (NFPA, 2013). Similar data are not available for VFFs.

Health. A number of factors including increasing obesity rates, job demands, and onduty cardiovascular disease (CVD) risk have escalated the concern about the health and fitness of firefighters in the US (Durand et al., 2011). Researchers have used a number of variables to assess the health of firefighter populations. When studying cardiovascular risk factors among 
200 firefighters across five suburban fire departments, Byczek, Walton, Conrad, Reichelt, and Samo (2004) examined both systolic (SBP) and diastolic (DBP) blood pressure, as well as cholesterol and weight. The authors do not elaborate whether the firefighters were career or volunteer. Among the sample, $23 \%$ of firefighters had high SBP, 14\% had elevated DBP which indicated one of the three stages of hypertension, and almost $25 \%$ had high cholesterol. These numbers were higher than those found among the general population in the same year (Byczek et al., 2004). Similar findings were reported by Soteriades and colleagues (2011), who also noted that firefighters with higher resting heart rate, DBP, and SBP were more likely to develop left ventricular hypertrophy.

Along with increased blood pressure and cholesterol, firefighters struggle with other areas of health as well. For instance, when examining aerobic capacity necessary for effective job performance, new recruits fell significantly below the necessary aerobic threshold for firefighting tasks (Roberts, O'Dea, Boyce, \& Mannix, 2002). Unfortunately, consensus regarding the minimum aerobic capacity required to successfully perform structural firefighting duties remain undiscovered (Durand et al., 2011). Furthermore, many departments do not require firefighters to maintain certain minimal physical capacities or follow certain exercise programs (Soteriades et al., 2005), and no mandatory national fitness requirements currently exist (Byczek et al., 2004). Without meeting the minimum level of aerobic capacity, firefighters are not only at risk themselves, but place the people they rescue at risk as well.

Obesity. According to the CDC, a BMI equal to or above 30 qualifies someone as obese. Similarly, a BMI at or above 25 equates to being overweight (CDC, 2012). For over 10 years, investigators have acknowledged the adverse association between health status and elevated BMI (Kales et al., 1999). Thus, multiple studies have been conducted to continue examining the rates 
of obesity amongst firefighters. When examining obesity, researchers explain that each firehouse and department is unique, so results may not generalize; however, investigators have consistently shown high rates of obesity among both VFFs and CFFs. For instance, Byczek and colleagues (2004) found that $82 \%$ of their firefighter sample had a BMI indicating overweight ( $44 \%$ of sample) or obesity ( $38 \%$ of sample), though they do not identify if the firefighters are career or volunteer. Soteriades and associates (2011) also found that $34 \%$ of their sample of career firefighters qualified as obese. Other samples of firefighters have shown a prevalence of more than $50 \%$ overweight and $35 \%$ obese (Durand et al., 2011). Over time, general rates of obesity and extreme obesity (BMI > 40) among firefighters have continued to increase (Soteriades et al., 2005). Individual firefighters also tend to get heavier each year, averaging 1.15 pounds gained per year they are on active duty. Moreover, firefighters with a BMI over 35 gained even more, averaging approximately two pounds gained per year. The increasing rates of obesity have also been associated with major CVD risk factors, and significantly lower overall strength and fitness (Poston et al., 2011a). Of those firefighters who passed away from CHD events while on-duty, approximately $90 \%$ of both CFFs and VFFs were overweight or obese according to their BMI (Geibe et al., 2008).

Anecdotally, many people argue that rates of obesity might be skewed, particularly in a cohort such as firefighters, because a person's BMI could be negatively affected if they have high muscle mass. Consequently, researchers have assessed obesity in a number of different ways, including BMI, body fat percentage, and waist circumference. Regardless of the method used, rates of obesity among firefighters remain the same (Poston et al., 2011a); in fact, BMI might actually underestimate obesity (Poston et al., 2011a), particularly in female firefighters (Jahnke et al., 2012a). In summary, rates of obesity among firefighters continue to be higher 
than those found in the general population (Poston et al., 2011a).

Consequences of Obesity. Negative factors associated with obesity, such as impaired movement, apnea-related fatigue, and lower levels of fitness, could cause firefighters who are classified as obese to be at a higher risk for occupational injury (Poston et al., 2011b). Body mass index was a significant predictor of injury-related absenteeism in 2010 and firefighter injuries, or efforts to prevent them, can cost firefighters, departments, society, and the insurance industry approximately $\$ 2.8$ to $\$ 7.8$ billion each year (TriData Corporation, 2005). Besides injury, obesity has also been related to higher SBP, DBP, and resting heart rate, as well as a group of other major CVD risk factors (Soteriades et al., 2005). Overall, obesity and lack of fitness among firefighters could be a key contributing factor to the high risk of on-duty CHD (Kales, Soteriades, Christophi, \& Christiani, 2007).

Nature of the job. Firefighters have a high stress job. Though only $5 \%$ of calls are fire related, sedentary time of firefighters is often filled with second jobs or overtime work, making exercise a low priority, especially since exercise is rarely mandated by departments (Durand et al., 2011). In addition, firefighters also use periods of inactivity as an opportunity to prepare and readily wait for the next call (Guidotti, 1992). Although down time might not be physically stressful for firefighters, there is also significant psychological stress related to firefighting due to the responsibility for the life and property of others (Guidotti, 1992), a pressure that does not wane in between calls. If firefighters are unable to successfully perform their duties, they put themselves, fellow firefighters, and others relying on them at increased risk of harm, injury, or death (Williford, Duey, Olson, Howard, \& Wang, 1999). Additionally, researchers have discussed the intense nature of firefighting, and the high aerobic capacity needed to effectively perform firefighting duties (Roberts et al., 2002). Because of the different stressors involved in 
firefighting, some have argued that it is "among the most strenuous occupations during periods of peak activity" (Guidotti, 1992, p. 1). Maintaining fitness as a firefighter could help with performing duties and preparing for life-threatening circumstances and stressful demands (Guidotti, 1992).

On-Duty Deaths. Although fire suppression only accounts for one to five percent of a firefighter's professional time each year, 32\% of firefighter deaths from 1994 to 2004 occurred during fire suppression duties (Kales et al., 2007). For a number of years, the leading cause of death for both VFFs and CFFs was sudden cardiac arrest (CDC, 2006; Fahy et al., 2012). It is believed that cardiac arrest among firefighters is caused by overexertion and stress (Fahy et al., 2012). Fifty-three percent of on-duty deaths were VFFs, and half of those deaths were specifically due to heart attacks (CDC, 2006). From 1994 to 2004, 39\% of total on-duty deaths were due to CHD (Kales et al., 2007). Researchers believe that if on-duty deaths were accounted for in these statistics, deaths specifically from CVD would be even higher (Byczek et al., 2004).

Potential Causes. There are a number of potential causes for the high risk of CVD among firefighters; for example, the cardiovascular stress experienced during a response, the carbon monoxide inhaled from smoke, or the extended inactivity broken by stressful alarms (Guidotti, 1992). Additionally, the fact that many firefighters are lacking the necessary levels of physical fitness to effectively perform their jobs could increase their risk of CHD while on-duty (Kales et al., 2007). The details and cardiovascular stressors of the job will be discussed later in this review. Regardless of the causes, it has been argued that increasing physical activity and total weekly exercise in firefighter populations could improve fitness and possibly decrease CVD risk (Durand et al., 2011).

Fitness and Firefighter Performance. To examine the relationship between fitness and 
performance of firefighter duties, researchers have taken firefighters through tasks related to fire suppression and measured different fitness parameters. Firefighters with lower body fat percentages and higher muscle mass were more likely to perform better during firefighting simulations, potentially showing the importance of fitness in job performance among firefighters (Williford et al., 1999). Unfortunately, many VFFs in one study had a $\mathrm{VO}_{2 \mathrm{Max}}$ that was so low, they would be unable to successfully perform their duties with little reserve capacity (Swank, Adams, Barnard, Berning, \& Stamford, 2000). Fitness is important in firefighting because it can provide resistance to unhealthy conditions (Peate, Lundergan, \& Johnson, 2002). A 16-week intervention significantly helped rookies with the lowest $\mathrm{VO}_{2 \mathrm{Max}}$ improve their aerobic capacity; however, the research did not follow up to see the effects of those changes on performance (Roberts et al., 2002). Overall, investigators found that firefighting stresses muscular endurace, strength, and aerobic power and endurance, so increases in firefighter health and wellness might lead to better performance (Rhea, Alvar, \& Gray, 2004).

Inactivity. One unique piece of firefighting comes in the downtime between calls. Though firefighting is strenuous when a call is received, stressful peaks come after extended periods of sitting and waiting (Guidotti, 1992). The effects of sedentary behavior can be seen in $\mathrm{VO}_{2 \mathrm{Max}}$ levels among firefighters, which suggests that firefighters were doing little activity or work to develop a minimum $\mathrm{VO}_{2 \mathrm{Max}}$ to effectively perform their job (Swank et al., 2000). In fact, four out of five VFFs studied did not engage in any physical activity outside of activities related to fighting fires. Sedentary behavior is a modifiable risk factor potentially related to increased risk of CVD (Tremblay, Colley, Saunders, Healy, \& Owen, 2010). During each shift, a firefighter often has prolonged sedentary stages while waiting for a call, which might increase firefighters' risk of CVD (Durand et al., 2011). Recent research has consistently found rates of 
inactivity among CFFs, where approximately $75 \%$ of those studied did not meet the CDC's minimum physical activity requirements of 150 minutes of aerobic exercise per week (Durand et al., 2011). More specifically, about 49\% percent of CFFs exercised three or fewer times per week, and only 22\% exercised five or more times (Durand et al., 2011). While it is understandable that rest would be desired in a high-stress job such as firefighting, increasing physical activity could improve risk profiles and overall fitness in firefighters (Durand et al., 2011) which is disccused in more detail below. The risks related to prolonged periods of sedentary behavior combined with firefighters' high levels of inactivity could negatively influence their levels of health and wellness, potentially hurting their job performance.

Though firefighters show high levels of inactivity and sedentary behavior, exercise is a useful tool in coping with stress. Research has shown that exercise might be effective in influencing the stress response in a couple of ways. One proposed idea is the Cross-Stressor Adaptation Hypothesis, which states that a stressor of proper duration and intensity, such as exercise, could help the stress response systems adapt; such adaptation then becomes evident during other stressful states (Sothmann et al., 1996). This adaptation is said to happen through one of two ways: habituation, a weaker response to stress from familiar stressors, or sensitization, a more effective response to a new stressor (Sothmann et al., 1996). In other words, exercise becomes a familiar challenge that affects the stress response when presented with a non-exercise related stressor (Lox, Ginis, \& Petruzzello, 2010). A second proposed explanation for the effectiveness of exercise in coping with stress is the Physiological Toughness Model (Dienstbier, 1989, 1991). According to this model, regular exposure to stress through exercise causes numerous physiological changes which lead to "adaptive performance in challenge/threat situations, emotional stability, enhancement of immune system function, and 
greater stress tolerance" (Lox et al., 2010, p. 281). Physiological reactions are moderated by an individual's appraisal of a situation as challenging (controllable, expect success) or threatening (uncontrollable, expect failure), but appraisals can be influenced by perceptions of arousal experienced in response to a stressor (Dienstbier, 1989, 1991). Essentially, the two responses are reciprocal; tough individuals have an adaptive response to stress, which leads to a positive perception, which increases the likelihood of appraising future stressors more positively (Lox et al., 2010). Regardless of the mechanism, research has shown a clear relationship between exercise and coping with stress. Thus, helping firefighters break their sedentary habits through creating a regular exericse regiment could help them manage the high levels of stress inate to their profession.

Firefighters and health and wellness. This section will discuss the literature reviewing firefighters' perceptions of health and wellness, the unique barriers that prevent firefighters from living healthier lives, interventions that have attempted to improve the health and wellness of firefighters, and recommendations found in the literature for firefighter populations.

Perceptions. Few researchers have sought to investigate firefighters' perceptions of health and wellness; however, those that have report some interesting results. For instance, when self-reported levels of fitness were compared to actual aerobic capacity, no significant association was found (Peate et al., 2002). Peate and colleagues argue this lack of relationship could be related to bias in self-report of physical activity. More recently, investigators examined accurateness of weight perceptions in CFFs, assuming that perception of weight can lead to decisions to change weight if the perception is non-ideal (Baur, Christophi, Tsismenakis, Jahnke, \& Kales, 2012). Eighty-nine percent of CFFs who fell within the normal weight category based on BMI correctly recognized themselves as normal; however, only $32.4 \%$ of overweight and 
$8.2 \%$ of obese CFFs correctly classified themselves (Baur et al., 2012). Baur and associates hypothesize that standards of weight, and therefore perceptions, could be skewed because such a high percent of the sample (86\%) were overweight or obese (2012). Knowing that firefighters' weight perceptions could be skewed is important because if CFFs are underestimating their weight, they might also be underestimating their health risk (Baur et al., 2012). Ultimately, understanding what type of education or intervention could successfully increase awareness is pivotal.

Barriers. Through a number of different studies, researchers have identified and hypothesized various barriers that prevent positive health behavior changes among firefighters. One point addressed in the literature is that consistent minimal fitness standards for firefighting have yet to be established (Durand et al., 2011; Rhea et al., 2004). This lack of standard makes creating exericse regimens to build proper strength and endurance difficult. Firefighters report that they are unsure what exercise is best for their work (Jahnke et al., 2012b).

The lack of national mandatory fitness standards for firefighters (Kales et al., 2007) means that each fire department is responsible for firefighter fitness, creating extreme variability (Byczek et al., 2004). Each department can individually determine their stance on fitness, exercise, and health screenings (Durand et al., 2011). Unfortunately, this individual responsibility results in a culture that does not prioritize physical fitness. Durand and colleagues eloquently explain, "The majority of US fire departments do not mandate exercise, lack regular exercise regimens, and do not require the maintenance of discrete physical fitness parameters after hire" (2011, p. 1753). In an attempt to create minimum standards, the NFPA released guidelines for medical surveilance, but these have not caught on, and many departments only require new recruits to complete baseline examinations (Soteriades et al., 2005). 
To learn more about the barriers faced by firefighters with regards to health, Jahnke and fellow researchers used focus groups to learn more about fire service personel perscpectives about firefighters' increased risk for disease and injury (2012b). A total of 332 career firefighters took part in the focus groups, along with officers, chiefs, and other personnel to learn more about their attitudes, perceptions, and opinions about the health of firefighters. Along with a lack of national standards for firefighter health and wellness, CFFs also report that departments do not mandate health and wellness, nor do they support health and wellness financially (Jahnke et al., 2012b). Consequently, resources to make positive changes are limited or non-existant. Unfortunately, there is also limited attention given to nutrition within fire departments, a factor significantly related to overall health (Jahnke et al., 2012b). In general, firefighter personnel in this study were aware of potential negative health implications related to firefighting, but social norms and other challenges to physical activity and nutrition made change difficult (Jahnke et al., 2012b). It is important to recognize that this sample consisted of only career firefighters, so the generalizability to volunteer firefighters is unclear. In addition, the authors mention that departments in the east, central, and west regions of the United States were represented, but give no other information about location. Therefore, confounding variables related to rural or suburban areas could have influenced the results.

The importance of leadership and departmental influence on firefighters has been echoed in other studies as well. For instance, Frattaroli and investigators also used focus groups to learn more about perspectives on health and wellness among volunteer firefighters, and to gather recommendations for how to reduce CVD risk through nutrition and phsical activity (2012). Participants in this study were volunteer firefighters currently volunteering in the state of Maryland; however, paid firefighters were invited to participate if they worked in a combined 
department that housed both volunteer and career firefighters (Frattaroli et al., 2012). Overall, firefighters reported unhealthy eating and activity behaviors, and identified the lifestyle of firefighting as a challenge to improving health behaviors (Frattaroli et al., 2012). To make positive change, firefighters expressed the importance of garnering support from the leadership in the firehouse, but also articulated how individual commitment might be weakened if a program was required (Frattaroli et al., 2012). It is clear that management and officers influence fitness norms within each fire house through their level of support (Staley et al., 2011).

Different generations of firefighters have also been shown to have varying expectations. Staley and partners found through focus group interviews that some prevention interventions may not be effective because young recruits are motivated by appearance rather than CHD risk (2011). The young recruits also had higher expectations of maintaining their fitness level than officers (Staley et al., 2011). Differing expectations and priorities for health between generations of firefighters could be a reason why interventions that are not uniquely designed based on personal goals are not effective. More research needs to be done to fully understand the relationship between firefighter preferences and intervention effectiveness.

Various other barriers have also been identified through research. For instance, a major barrier identified by CFFs was the food environment within the fire house (Jahnke et al., 2012b); the options are limited and tend to be predominantly unhealthy (Frattaroli et al., 2012). Also, because of the uncertainty of when a call might come in, many firefighters also reported sleep problems as a barrier to health (Jahnke et al., 2012b). Within the VFF culture, firefighters have to manage their role in a day job and work for the fire department, which can be a major barrier to eating well or engaging in physical activity (Frattaroli et al., 2012). The potential options for VFFs are also limited by their budgets because volunteer fire departments often have fewer 
resources (Scanlon \& Ablah, 2008). In some cases, departments may require physical examinations, but do not pay for them; even if departments do pay, VFFs are responsible for the cost associated with follow up tests or care. Finally, VFFs report lack of CVD education as another major barrier to change. While some of these barriers are faced by a number of other people as well (e.g., multiple jobs, lack of time), it is important to recognize the combination of factors that potentially influence the health behaviors of firefighters, and how firefighters might successfully navigate those influences.

Staley and colleagues (2011) articulated further barriers and facilitators to firefighter involvement in physical training by examining varying levels of influence based on the Social Ecological Model. Authors recruited firefighters from four career departments in the southeastern US and conducted key informant interviews, department observations, and focus groups to better understand expectations and cultural factors that influenced physical fitness. Multiple interviews took place with each of six firefighter informants to learn more about the language and culture of firefighting. Researchers then observied the daily routines of the firefighters, and rode along during emergency calls. Finally, six focus groups were conducted at four urban, career fire departments where the key informants worked. In total, 64 participants took place in the focus groups. Results were thematized and coded using intrapersonal, interpersonal, and organizational levels. At the intrapersonal-level, motivation was most highly rated, followed by fitness beliefs, nutrition and diet, and CHD and sudden cardiac death knowledge. Interpersonally, the most important factors were crew-level dependability, social cohesiveness, and fitness level norms of crew and captains. At the organizational level, participation strategies were most often cited. Lastly, crucial organizational influences were the fitness norms and support from management (Staley et al., 2011). It is clear that multiple factors 
influence perceptions, preferences, and behaviors of firefighters.

Interventions. Regardles of the different barriers to health and wellness, some researchers chose to develop intervention studies in an attempt to help firefighters foster healthier lifestyles. For instance, after implementing a 16-week exercise program specifically designed for physical fitness in firefighters, rookies showed significant improvements in muscle endurance, $\mathrm{VO}_{2 \mathrm{Max}}$, and flexibility (Roberts et al., 2002). Unfortunately, this intervention did not significantly change the body weight or BMI of the participants. However, rookies that started with the lowest $\mathrm{VO}_{2 \mathrm{Max}}$ before training showed the greatest improvements over the 16-week intervention (Roberts et al., 2002). Long-term effects of this intervention were not discussed, making it unclear if positive changes were longlasting. A different intervention was launched in 2003 by the National Volunteer Fire Council called the Heart-Healthy Firefighter Program, which includes different health screenings in an attempt to encourage better nutrition, fitness, and health to prevent heart attacks (Fahy et al., 2012). Since its inception, the program has evolved to include opportunities for earning rewards as each firefighter meets fitness goals, education (healthy recipes, heart disease risks, etc.), resources for developing programs in a department, and smoking cessation programs (NVFC, 2013). It is unclear if any analysis has been done to evaluate the effectiveness of this program or how often it is used by departments across the country.

Perhaps one of the most in-depth health interventions with firefighters to date is the Promoting Healthy Lifestyles: Alternative Models' Effects (PHLAME) study done by Moe and colleagues (2002). Because investigators have consistently found that the most common cardiac risk factor among firefighters with CHD is insufficient regular exercise, Moe and associates (2002) began by outlining a health behavior intervention based on motivational interviewing 
(MI) and social learning theory (SLT). The study took place in Oregon and Washington, and firefighter participants were separated into three groups: (a) one-on-one, (b) team, and (c) control or usual care (Moe et al., 2002). In the one-on-one condition, firefighters were provided counseling based on the Transtheoretical Model (Prochaska \& DiClemente, 1992) to individualize interactions and MI (Miller, 1985). Individual meetings lasted an hour and took place about every week for three or four sessions. Relationships between each firefighter and health, values, and lifestyle were discussed to help ease the process of behavior change with the firefighters (Moe et al., 2002). The team intervention part of PHLAME was based on Bandura's SLT (as cited in Moe et al., 2002), and used the "team" nature of firefighting to help each individual initiate behavior change. Each piece of the curriculum was taught by a shift member with proper training, and a total of 11 sessions each lasted approximately 45 minutes. Meetings involved delivering health test results, team activities that taught application of concepts, problem-solving activities, and friendly competitions (Moe et al., 2002). Those firefighters assigned to the control or usual care group received their test results and a short interpretation; no other intervention or education was provided (Moe et al., 2002).

Since its implementation, a number of follow-up studies have discussed the findings and outcomes of PHLAME (Elliott et al., 2004; Elliot et al., 2007; Ranby et al., 2011). In the first review of findings, Elliot and collaborators (2004) found that firefighters in the one-on-one group decreased their dietary fat intake $($ beta $=-0.26, \mathrm{p}=0.008)$ and had fewer feelings of depression compared to the other two groups (beta $=-0.72, p=0.017$ ). Firefighters in the teambased group increased their personal exercise habits (beta $=0.99, \mathrm{p}=0.008)$, increased rates of cohesion $(\mathrm{beta}=.66, \mathrm{p}=0.024)$, and improved diet $($ beta $=0.76, \mathrm{p}=0.009)$ and exercise $(\mathrm{beta}=$ $1.19, \mathrm{p}=0.001)$ habits compared to the control group (Elliot et al., 2004). Overall, both the team 
and individual approach seemed well-received by the firefighter participants, and both showed significant but unique improvements in areas of health and wellness (Elliot et al., 2004). A few years later, a follow up review showed similar benefits for the two experimental groups. Both the one-on-one and team groups increased their vegetable and fruit consuption $(\mathrm{p}<0.01$ team, $\mathrm{p}$ $<0.05$ one-on-one), showed less weight was gained ( $\mathrm{p}<0.05$ for both), and improved general well-being ( $\mathrm{p}<0.01$ for both) compared to the control group (Elliot et al., 2007). In general, the PHLAME intervention was shown to have a positive influence on health behaviors, increased well-being, and decreased amount of weight gained (Elliot et al., 2007). Unfortunately, effect sizes are not available for the analyses conducted in these studies.

When the investigators examined why the PHLAME study had a positive effect, a number of mediating variables were identified. First, firefighters in the team group showed that coworker norms and knowledge of the benefits of fruits and vegetables improved in the team condition over the control group (Ranby et al., 2011). In addition, the imapct of peer-lead education in the team group may have also had a positive effect (Elliot et al., 2007). Ultimately, it seems as though support from coworkers for making positive health change, emphasizing the benefits of a healthy lifestyle, and fostering teamwork during behavior change is most effective (Ranby et al., 2011). In all of their reviews, however, qualitative analysis of the firefighters' experience was not considered. Though this study has been instrumental in understanding what aspects of health behavior might be most accepted by firefighters, much can still be learned about the motives and hesitations of firefighters that are or are not effective in making healthy change. In fact, researchers acknowledge that more work needs to be done to determine the interventions that would be most effective in enhancing firefighters' health and wellness (Poston et al., 2011). 
Recommendations. Regardless of the focus of the study, many researchers outline different recommendations for firefighters based on current knowledge of health and wellness, and intervention effectiveness. Although minimum standards for firefighter fitness are not fully understood, it is evident that firefighters need high levels of muscular endurance, muscular strength, and cardiovascular endurance to be effective in their jobs, and an exercise prescription specifically for this population could help (Rhea et al., 2004). In order to control firefighters' poor cholesterol numbers, Byczek and colleagues recommended weight reduction as a potentially effective way to initially increase HDL levels, while simultaneously lowering LDL's, triglycerides, and overall cholesterol (2004). Similarly, risk profiles in CFFs could be improved by increasing total physical activity each week (Durand et al., 2011). Because researchers have discovered that overweight and obese firefighters might underestimate weight and weight-related risks (Baur et al., 2012), education on weight status and associated hazards could be beneficial.

On a departmental or administrative level, it has also been suggested that invesments should be made to develop programs that encourage physical fitness to help reduce decreases in $\mathrm{VO}_{2 \mathrm{Max}}$ related to age and inactivity (Swank et al., 2000). A program similar to the 16-week exercise intervention introduced by Roberts and colleagues could be a valuable starting point (2002). Against the preferences of individual firefighters, some have also recommended that departments should consider requiring annual fitness and medical exams to help reduce cardiovascular disease risk, including mandated involvement in a fitness program (CDC, 2006). Additionally, screenings for CVD risk factors have been suggested to departments and physicans caring for firefighters (Soteriades et al., 2005). Along with screenings, departments should also reserve time for physical training in each firefighter's schedule to promote prioritizing of health and wellness (TriData Corporation, 2005). Finally, because fire houses, particularly volunteer 
departments, work on such limited budgets, departments could hire fitness consultats, seek donations, or request discounted memberships from local or nearby gyms for firefighters in the area to encourage outside phsyical fitness if the fire house cannot provide an in-house gym (TriData Corporation, 2005). While these recommendations are all helpful, until investigators have a better understanding of the facilitators of, and barriers to, health behavior among firefighters, it is unclear how effective any of the aforementioned suggestions might be.

Volunteer firefighters versus CFFs. Although VFFs make up a vast majority of firefighters in the US, limited studies that look at health, wellness, and fitness of firefighters chose to examine a volunteer cohort (Frattaroli et al., 2012). Recently, however, scholars have realized the importance of VFFs, and have included them more in the research. One reason research needs to learn more about VFFs is because they have access to fewer resources than career firehouses (Frattaroli et al., 2012), meaning that even though they are interested in receiving education on living a healthier lifestyle (Scanlon \& Ablah, 2008), they might not have the means to do so.

Rates of obesity in VFFs are just as high, if not higher, than CFFs, showing as high as $41 \%$ overweight and $35 \%$ obese (Scanlon \& Ablah, 2008). These numbers were supported more recently, where $75 \%$ of VFFs had a BMI over 25 (Poston et al., 2011a). Unlike CFFs, VFFs might meet the minimum CDC phsyical activity requirements as they self-report an average of 5.92 hours of exercise each week (Scanlon \& Ablah, 2008). On the other hand, approximately six hours of exercise a week might not convert to aerobic capacity. When compared to the 12 MET physical estimate required to be effective while firefighting, less than $25 \%$ of VFFs could meet or exceed that minimum requirement, compared to almost $39 \%$ of CFFs, showing that aerobic fitness and endurance could be worse in VFFs (Poston et al., 2011a). Volunteer 
firefighters are aware of the CVD risk assiciated with firefighting, and almost $76 \%$ surveyed accurately named heart attacks as the number one cause of on-duty deaths (Scanlon \& Ablah, 2008). However, VFFs reported that they struggled decreasing their CVD risk because they did not have sufficient education on CVD or had trouble receiving appropriate healthcare (Scanlon \& Ablah, 2008). In addition, over $90 \%$ of VFFs in the study either strongly or somewhat showed interest in participating in a department-run fitness program, and strongly or somewhat agreed that departments should be more active in educating VFFs about risks associated with firefighting (Scanlon \& Ablah, 2008). While this information is helpful in gaining a better understanding of VFF statistics, it is important to understand the limited generalizability of the findings. Scanlon and Ablah used a nonvalidated questionnaire to learn more about the cardiac risk factors, and motivation to resolve those risk factors, among volunteer firefighters in Nassau and Suffolk counties in Long Island, NY. The information gathered is helpful, but because the VFFs in their sample represented a very small area of NY, the results might not apply to other, more rural areas of the country.

When comparing risk of death from CHD while on-duty, VFFs and CFFs differ slightly. Volunteer firefighters have lower risk than CFFs during fire suppression, physical training, and fire-station duty; however, VFFs have higher risk during alarm response and emergency medical services (Kales et al., 2007). Despite these differences, both groups showed the highest risk of death from CHD during fire suppression (Kales et al., 2007). Further research showed that the prevalence of cardiovascular risk factors in VFFs and CFFs did not differ significantly, and that a vast majority of firefighters, both VFFs and CFFs, who experienced CHD on-duty had a BMI in the overweight or obese range, whether they passed or survived (Geibe et al., 2008). Furthermore, the number of VFFs who died while on-duty was $53 \%$, higher than the $32 \%$ of their 
CFFs counterparts (CDC, 2006). As mentioned previously, the leading cause of death for both CFFs and VFFs included sudden cardiac death, (CDC, 2006). Their higher prevalence in the work force, added stress of balancing multiple roles, and worse levels of health and wellness make VFFs a population that warrants more attention in the literature.

\section{Appalachian Culture}

Demography. Appalachia includes a total of 13 states. Portions of 12 states including New York, Pennsylvania, Ohio, Maryland, Virginia, Kentucky, Tennessee, North Carolina, South Carolina, Georgia, Alabama, and Mississippi (ARC, 2013) are considered part of Appalachia. West Virginia is a unique state within the Appalachian region because it is the only state wholly included in Appalachia. Forty-two percent of the Appalachian population is considered rural, significantly lower than the $20 \%$ of the US population that is considered rural. Further, $18 \%$ of Appalachian residents lived in poverty as of 2008, a number that dropped almost in half from 50 years earlier when $33 \%$ of residents were poverty stricken.

Appalachian Health and Wellness. With regards to health, Appalachia tends reports some of the highest rates of obesity and diabetes when county-by-county comparisons are made; more than $80 \%$ of the counties in Appalachia report high rates of obesity and diabetes (CDC, 2007). Appalachia is home to approximately 24.8 million people (ARC, 2010); however, almost $44 \%$ of the Appalachian population is considered obese (Wewers et al., 2006). The high rate of overweight and obese individuals in Appalachia is important because the most significant risk factor for diabetes among Appalachian residents was obesity, followed by physical inactivity (Serrano, Leiferman, \& Dauber, 2007). Obesity is also related to a number of other health problems, and in one study, only $36.8 \%$ of overweight or obese adults had less than two cardiometabilic abnormalities such as hypertension, high triglycerides, high fasting glucose, high 
cholesterol, diabetes, high c-reactive protein, or insulin resistance (Blake, Shankar, Madhavan, \& Ducatman, 2010). In general, Appalachia tends to report higher mortality rates and higher rates of serious disease than the national average (ARC, 2010).

Along with poor health, rates of physical activity in Appalachia also tend to be low. In an attempt to learn more about physical activity in Appalachia, Zizzi and colleagues (2006) surveyed 1,239 church-going men and women about physical activity and other characteristics. Overall, $48 \%$ of participants reported no regular weekly exercise; $12 \%$ further stated that poor health was a barrier to physical activity. Interestingly, the researchers found that barriers to physical activity were low, with only $33 \%$ of respondents claiming a lack of time to be physically active; however, further analysis showed that barrier did not mediate physical activity behavior. Overall, age, gender, self-efficacy, and obesity proved to be primary correlates of physical activity. More specifically, physical activity declined as age and obesity increased; as income and self-efficacy increased, likelihood of meeting physical activity guidelines increased; and men were more likely to meet the fitness standard than women. Common activities of those participants who did meet physical activity guidelines included walking, housework, gardening, strength training, and biking; these activities could be effective if used during interventions targeting physical activity in Appalachia. A clear limitation of the study is the potential lack of generalizability as the sample was of church-goers who reported higher income and education levels than general Appalachian residents, and was substantially older than the average firefighter population. However, this information is useful as a foundation to understanding physical activity rates in population sub-sets in Appalachia.

The weaker economy of some areas within Appalachia could also play an important role in health and wellness. Specifically among women, those in Appalachian counties with "weaker 
economies" showed higher odds of being overweight or obese, completing less education, not having health insurance, and poor mental health when compared to women in counties with “strong economies" (Short, Oza-Frank, \& Conrey, 2012). Similar results were found between women in Appalachia compared to non-Appalachia women, but Appalachian women also had a higher likelihood of reporting no health insurance, fair or poor health, no routine check-up in the last year, and history of hypertension (Short et al., 2012).

Overall, Appalachia serves as a unique setting for studying health factors because of the unique combination of individual and environmental factors that influence the health of Appalachian residents. Researchers have noted the need for continued research in Appalachia, specifically with regard to physical activity. Kruger and colleagues explain, "The magnitude of the problem of physical inactivity in Appalachia, coupled with the unique cultural, economic, and environmental characteristics of the region, warrants special attention to the barriers to and facilitators of successful PA interventions" (2012, p. 144). For the same reasons, other aspects of health, such as nutrition, should also continue to be examined amongst the populations in Appalachia so effective interventions can then be developed based on the findings. Furthermore, understanding perceptions of Appalachian residents with regards to physical activity and nutrition can help guide successful interventions, as our understanding of perceptions and programs within this region is still lacking (Kruger et al., 2012).

Barriers to Health. A number of barriers to health behavior exist in rural areas such as Appalachia. For instance, among rural women, less education, older age, less social support from family and friends, and not seeing others exercise were associated with sedentary behavior (Wilcox, Castro, King, Housemann, \& Brownson, 2000). Furthermore, higher income residents in suburban areas were more than twice as likely than lower income residents in rural areas to 
meet minimum physical activity recommendations (Parks, Housemann, \& Brownson, 2003). This relationship could be related to access, as the more places available for a person to exercise, the more likely a person is to meet the physical activity recommendations (Parks et al., 2003). Lower income is also related to lack of health insurance coverage, and residents of rural counties tend to be more likely to be without health insurance than suburban residents (Eberhardt \& Pamuk, 2004). Even for residents that do have health insurance, people living in rural areas often live farther away from health care resources, making it difficult to access useful resources (Glasser at al., 2003). In addition, promoting even the most common forms of physical activity, like walking, can be challenging in rural settings because streets are poorly connected, sidewalks are rare, street lighting is inadequate, and car travel is the preferred method of transportation (Saelens, Sallis, \& Frank, 2003). Such environmental barriers might prevent physical activity interventions from being effective in rural settings (Zizzi et al., 2006). Zizzi and colleagues (2006) summarize this information well by explaining that a number of factors could contribute to the health problems faced in rural settings such as Appalachia, including lower income, less education, and living further away from health-care resources.

Common barriers seen outside of Appalachia have also been shown to be prevalent for Appalachian residents. For instance, "negative experiences while engaging in PA, fatigue from time spent at work, and time taken by other commitments reducing their desire and ability to engage in PA" were all barriers reported by participants in a study of rural Appalachian residents (Kruger et al., 2012, p. 146). In addition to those barriers, however, participants also reported barriers including challenging physical environments, lack of opportunities for PA in the community, long travel distances to destinations, and competing priorities; barriers Kruger and colleagues identify as more specific and unique to the Appalachian region (2012). Because of 
the economic stresses faced by many areas of Appalachia, the barriers listed above might also be exacerbated because of the persistent poverty those residents face. Keeping these barriers in mind, effective interventions to increase physical activity among Appalachian residents should then focus on designing programs that keep extended families in mind, advertise programs well, focus on health over appearance, and incorporate culturally relevant activities (Kruger et al., 2012). Programs with a group- rather than individual-focus might also be more widely accepted in Appalachia because of the community focus (Kruger et al., 2012).

West Virginia. As mentioned previously, West Virginia is a unique state in Appalachia because it is the only state that is not a part of another region. According to the United States Census Bureau, the population of West Virginia in 2012 was 1.855 million residents, 20.7\% of whom are under 18 , and $16.8 \%$ of whom are 65 years old or older. Citizens of West Virginia are predominantly white (94\%), while African-Americans represent 3.5\% of the population and Hispanics or Latinos make up $1.3 \%$ of the people in the state. The median household income from 2007 to 2011 was $\$ 39,550$ in West Virginia, almost $\$ 15,000$ lower than the national average for the same time frame. Additionally, $17.5 \%$ of West Virginia residents live below the poverty line; three percent more than the national average of $14.3 \%$. Of people 25 years old or older in the state, $82.6 \%$ hold a high school diploma, but only $17.6 \%$ have a bachelor's degree or higher, compared to a $28.2 \%$ national rate, showing that educational levels in West Virginia are lower than the national average. As of 2010, West Virginia averaged 77.1 persons per square mile compared to the national average of 87.4. West Virginia is repeatedly one of the most obese states in the country, and in $2012,33.8 \%$ of the population was obese, up more than $1 \%$ from only two years earlier (CDC, 2012). In West Virginia and five other states, obesity prevalence in $70 \%$ counties was in the top quintile $(\geq 30.9 \%$; CDC, 2009). When examining the 
West Virginia WISEWOMEN population, numbers become even more concerning. Rye and colleagues (2009) found that almost $84 \%$ of their participants, women age 40 to 64 , were overweight or obese. In West Virginia, males are more likely to be obese than females (Amarasinghe, D'Souza, Brown, Oh, \& Borisova, 2009), meaning that the number of males who are overweight or obese in WV in the same age range could be higher than the $84 \%$ reported by Rye and colleagues. Obesity is not the only health problem plaguing West Virginia; approximately $62 \%$ of counties in West Virginia and Tennessee, when combined, were in the top quintile of diabetes prevalence (CDC, 2009). Furthermore, $81 \%$ of counties in West Virginia, Kentucky, and Tennessee were in the top two quintiles for both diabetes and obesity (CDC, 2009).

Rates of obesity among both adults and adolescents in West Virginia have led to numerous interventions including legislative efforts to battle obesity (Harris et al., 2010). One such intervention at the state level that targets childhood obesity is the Healthy Lifestyles Act, which led to the creation of the Office of Healthy Lifestyles (Harris et al., 2010). The five mandates of the Act required that schools provide healthy beverages, physical education, fitness testing, BMI measurements, and health education and assessment. Also using the schools as a venue to encourage health behavior, Rye, Tompkins, McClure, and Aleshire (2008) worked with high school students and teachers to increase opportunities for physical activity. After two years, $73 \%$ of student participants decreased their reported number of barriers to physical activity (Rye et al., 2008).

A number of relationships exist between obesity and other factors among West Virginia residents. For instance, students are less likely to be obese than people who have been out of work for a year, or those who are unable to work (Amarasinghe et al., 2009). Similarly, as 
education goes up, the likelihood of being obese goes down by 3\% (Amarasinghe et al., 2009), but less than 20\% of WV residents hold a college degree (US Census Bureau, 2012). Furthermore, smokers were less likely to be obese, but those with a sedentary lifestyle were more likely to be obese. Not surprisingly, obesity decreases expected self-assessed health (Amarasinghe et al., 2009). Finally, drive-time to and from work also impacts the chance of obesity, as every minute increase in travel time leads to an increase in the probability of obesity by $0.4 \%$. West Virginia residents likely have to travel farther for employment opportunities, or for normal daily activities such as shopping (Kruger et al., 2012), so this information is particularly relevant. Overall, factors related to obesity include unemployment, lower education levels, sedentary behavior, and increased travel time, issues popular in a rural state such as West Virginia. Therefore, when examining health behavior patterns such as physical activity and nutrition, researchers must keep in mind the myriad factors that influence obesity and how they interact reciprocally.

\section{Social Ecological Model (SEM)}

Since the 1980's, health promotion research has noticed a distinct shift in individualfocused analysis of health, to more broad, environmental-focused health promotion strategies (Staley, 1996). In fact, it is said that ecological models are now "commonplace in public health discourse", and SEM training is becoming more emphasized (Golden \& Earp, 2012, p. 365). According to Sallis and Owen (2002), ecological models of health behavior are

"models proposing that behaviors are influenced by intrapersonal, sociocultural, policy, and physical-environmental factors; these variables are likely to interact, and multiple levels of environmental variables are described that are relevant for understanding and changing health behaviors." (p. 463) 
By considering multiple levels of influence with regard to health behavior, it is believed that more comprehensive and effective interventions can be developed (Sallis, Owen, \& Fisher, 2008).

To gain a better understanding of multiple factors that can influence health behavior, some researchers have adopted the social ecological model, which is "an overarching framework, or set of theoretical principles, for understanding the interrelations among diverse personal and environmental factors in human health and illness" (Stokols, 1996, p. 283). In other words, SEM "offer[s] a theoretical framework for understanding the dynamic interplay among persons, groups, and their sociophysical milieus" (p. 283). While other theories in health behavior research tend to focus on one aspect of health, such as the individual person or specific behavior, SEM emphasizes the connections and interdependence of situational and personal factors rather than focusing solely on biological or environmental characteristics (Stokols, 1992, 1996). Because SEM considers so many important factors of health behavior, it is often multidisciplinary, and uses diverse methodologies and multiple levels of analysis.

When utilizing an SEM framework, it is suggested that investigators begin by identifying aspects of the environment that promote both personal and collective health and well-being (Stokols, 1992). It is important to remember that physical, emotional, and social health are all important aspects of healthfulness within the ecological perspective. An effective analysis using SEM would then investigate the interplay between individual and group behaviors, and resources and barriers within sociophysical environments.

The core concept of SEM is that behavior is driven by multiple levels of influence (Sallis et al., 2008). Four core principles of ecological models include: the belief that there are multiple influences on health behavior, influences interact across different levels, models are most 
effective when behavior-specific, and effective behavior change is most likely from multi-level interventions. When individuals are educated and motivated to make healthy choices, social norms and support back healthy choices, and environments and policy encourage healthy choices, SEM believes behavior change will be maximized (Sallis et al., 2008). Without environments and policies that support healthy behavior, motivation and skill education are insufficient to produce individual behavior change.

Models of SEM. In 1977, Urie Bronfenbrenner proposed a new perspective to studying and understanding human development, which he referred to as "the ecology of human development" (p. 514). In his article, Bronfenbrenner discussed the importance of the interaction between a developing person and their changing environment, which consists of four successive levels. Microsystems refer to the immediate setting surrounding a person such as the home, workplace, or educational setting. One level removed lays the mesosystem, which houses the "interrelations among major settings containing the developing person at a particular point" (p. 515). The mesosystem is essentially a system of microsystems that might include interactions between peer groups, family members, and church acquaintances, for example. Beyond the mesosystem is the exosystem that includes social structures outside of the person, but which impact the settings that immediately surround the person. Examples of exosystem factors include neighborhoods, government, and the mass media. Finally, the most outer layer is the macrosystem, referring to institutional patterns of culture or subculture, which exist within economic, legal, education, political, and social systems. Those patterns created at the macrolevel create blueprints that guide structures and activities occurring at lower levels of the model. After defining the different levels of influence, Bronfenbrenner (1977) also outlined properties and propositions about each level. At the micro-level, reciprocity is key. In other 
words, the individual exerts influence on their environment, and the environment exerts influence on the individual. Furthermore, it is pivotal to recognize the importance of every person within the social system of a setting, rather than focusing only on one individual, as each person influences the other. Because multiple parties must be considered, understanding the possible subsystems that then exist is also important. Finally, at the micro-level researchers should account for the features of the environment that could impact social processes. Important elements to consider at the meso-level include the interactions between settings, subsystems or higher order effects that might exist across settings, and transitions or changes within an ecological system that naturally occur throughout a person's life. Within the exo-level, researchers must remember to account for influences beyond the individual and immediate setting to account for the larger context that might be influencing the lower levels. Lastly, the macro-level provides researchers an opportunity to examine different ways of restructuring current systems or connecting systems in unique ways (Bronfenbrenner, 1977).

While Bronfenbrenner's model is often cited, a similar perspective was created by McLeroy, Bibeau, Steckler, and Glanz (1988). According to their approach to health promotion programs, behavior is determined by five factors. First, intrapersonal factors are characteristics of a person. Second, interpersonal processes and primary groups include social networks and support, similar to Bronfenbrenner's microsystem. Next, institutional factors relate to social institutions, including formal and informal rules and regulations. Fourth are community factors that refer to relationships between institutions, organizations, and informal networks, mirroring Bronfenbrenner's mesosystem. Finally, public policy are policies and laws at the local, state, and national level (McLeroy et al., 1988). Part of the justification for creating a new approach to SEM was because previous ecological models lacked enough specificity to conceptualize 
effective interventions or understand certain problems.

Similar to Bronfenbrenner, McLeroy and colleagues (1988) outline important processes at each level to consider when conducting research using SEM. At the interpersonal level, social relationships are key, and play a large part in social identity. Health promotion interventions focusing on this level most focus on using social influence instead of changing group norms to change health behavior. Regardless of the focus, interpersonal interventions should try to change the nature of social relationships to those that support healthy behavior.

The organizational level is of great importance because people spend one-third to onehalf of their lives in organizational settings (McLeroy et al., 1988). Therefore, organizational changes can help support long-term behavior change, and can significantly influence the adoption, implementation, and maintenance of programs. Community influence is also significant in health behavior because power structures within each community can serve to support or block health behavior and program implementation. Most health services are offered through community organizations, so relationships between individuals and the community can significantly impact health behavior. Finally, public policy can help increase awareness of health and policy issues, educate people on policy development, and advocate for people to participate in the process. Overall, the goal of public policy is to help mediating structures meet the needs of members (McLeroy, 1988).

Regardless of the specific SEM framework used, the purpose of ecological approaches is to bring awareness to the different causes within an environment that influence behavior, and develop interventions based on that awareness (McLeroy et al., 1988). If using an SEM approach, it is pivotal that researchers rely on active involvement of target populations to help define specific problems, identify what needs to be changed, and design effective interventions 
(McLeroy et al., 1988), something lacking within the firefighter health and fitness literature.

Strengths. Taking a social ecological approach to understanding health behavior offers numerous strengths. First, SEM relies on both behavioral change and environmental enhancement to address behavior change, rather than focusing on only one variable (Stokols, 1996). Focusing on environmental influence at the community, organization, and policy levels, rather than investigating purely individual or social influences, is a unique aspect of ecological models (Sallis et al., 2008). This focus on multi-level influence also helps broaden the options that are available for interventions. Additionally, SEM emphasizes cross-level analyses, investigating two or more levels of health behavior to gain a broader understanding of influences and outcomes. Consequently, proverbial "blind spots" inherent to approaches that only measure behavioral or environmental variables are avoided because the interaction of elements at multiple levels is considered.

Limitations. As with any approach, SEM has some limitations. For an effective SEM intervention to be developed, cooperation from professionals across different disciplines is key (Stokols, 1996). Also, because research based in SEM is interested in multiple levels of influence, studies and interventions are often expensive and complex, meaning studies can become impractical to implement. Methodologically, a major challenge presents itself when trying to manipulate interactions between personal, social, or community characteristics (Sallis et al., 2008). There is also a lack of information regarding the influence of broader levels, how that influence operates, and how factors interact across levels (Sallis et al., 2008). In fact, support has been shown for the idea of multi-level influence on behavior; however, the interactions across levels have not gained consistent support.

While there has been a call for interventions to rely more on multilevel approaches, 
including the social, institutional, and policy levels, recent literature reviews have shown that nearly two-thirds of health related articles target only one or two levels (Golden \& Earp, 2012). Most literature continues to focus attention at the individual level. Specific barriers to the SEM approach that could cause this could include lack of training and resources for health educators, poorly validated measures of change, and insufficient funding, all of which echo the limitations inherent to SEM (Golden \& Earp, 2012). Despite the possible limitations, an SEM approach is beneficial because different aspects of a person and their surroundings are considered. In the culture of firefighting, no firefighter acts independently; firefighters are influenced by their peers, the leadership of the department, other local departments if necessary, and even local, state, and federal government with regards to their budget, resources, and requirements. Therefore, using SEM as a means to understand the context of firefighting is quite useful despite the difficulties SEM presents.

SEM in Physical Activity. For years, it has been widely accepted that multiple determinants influence adult physical activity at the personal, social, and environmental level (Trost, Owen, Bauman, Sallis, \& Brown, 2002). In 2002, a meta-analysis of the literature on physical activity and health promotion updated the summary of factors that can affect adult physical activity behavior. At the personal level, demographic and biological factors (e.g., age, gender, socioeconomic status, and educational attainment); psychological, cognitive, and emotional factors (e.g., barriers to physical activity, enjoyment of physical activity, intentions, normative beliefs, psychological health, and self-efficacy); and behavioral attributes and skills (e.g., decisional balance, past exercise behavior, dietary habits, etc.) were the important factors identified (Trost et al., 2002). One level further removed are the social and cultural factors, among which one of the most important characteristics is social support. Other important 
determinants include past family influence, physician influence, and group cohesion. Finally, the authors discuss the important physical environment factors which exist at two levels. First, individual level environmental factors are determinants such as having exercise equipment at home, and perceived or actual access to facilities. Second are the community level environmental influences which includes hilly terrain, enjoyable scenery, and neighborhood safety. Trends in the literature show that a broader perspective is being taken to understand ecological and health promotion models that effectively influence physical activity behaviors (Trost et al., 2002). Using SEM as a framework for studying health behavior provides researchers and professionals a more comprehensive understanding of the multiple variables that can influence an individual's various health behaviors.

SEM in Firefighting. Limited research has been conducted exploring the levels proposed in SEM and the culture of firefighting to gain a better understanding of health behaviors. One exception is Staley and colleagues who used SEM as a conceptual framework to guide their examination of previous fitness interventions and the unique culture of firefighting to gain a better understanding of multilevel influences that might explain why past interventions have not been effective in helping firefighters become more fit (2011). The authors identified numerous barriers and facilitators to physical fitness at the intrapersonal (e.g., motivation, beliefs, nutrition), interpersonal (e.g., social cohesiveness, crew dependability, crew and captain norms), and organizational (e.g., participation strategies, work environment factors, management support or lack of support) levels. While this information provides support for using SEM to guide understanding of the various factors influencing the health of firefighters, the participants in this study were all career firefighters located at four houses in the southeastern United States (Staley et al., 2011). Therefore, the generalizability of these findings to firefighters in other 
regions of the country, or to volunteer firefighters who work within a different culture, could be limited. It is important for future research to continue using SEM to guide further study of other multi-level factors that could be influencing firefighters in other parts of the U.S.

Based on other information reviewed, a further summary using SEM as a foundation can be done. First, at McLeroy and colleague's intrapersonal level exists each individual firefighter (1988). At this level, barriers to health beahvior could include attitudes toward exercise, such as believing that physical activity is a waste of energy. Motivation might also be a barrier at the intrapersonal level, as reported by Staley and colleagues (2011); firefighters might simply lack motivation to begin or maintain certain health behaviors.

One level higher is the interpersonal or micro-level, which includes the interactions between the firefighters, as well as the influence of their home life and other jobs (if applicable). Here, barriers to health behaviors for firefighters might include norms regarding physical activity or nutrition behavior (for example, not exercising because no one else in the department exercises or sees value in physical activity), availability of nutritious or unhealthy options at each department, or the fitness levels of firefighters in leadership positions. Leadership can play an influential role, as firefighters might believe that if the captain does not need to maintain certain fitness standards, why should they?

The institutional, or exo-level, could also create numerous barriers to health behavior for firefighters. For instance, since no national standards exist for physical activity, each department is responsible for creating their own fitness standards. If a department fails to do so, firefighters might not feel they have the support from their leadership to engage in physical activity. This also relates to the next level above, public policy, which influences firefighters by neglecting to create clear, mandatory fitness standards. In addition, public policy and community levels could 
both be related to financial burdens placed on fire departments, volunteer departments specifically, which limits available options for healthy behavior.

Interventions Based on SEM. Since the application of the SEM approach to health behavior, numerous interventions have been created to address multiple levels of influence on behavior. Perhaps two of the most successful and well-known interventions addressed smoking cessation and diabetes management (Sallis et al., 2008). To help stop individuals from smoking, interventions at multiple levels were used. At the individual level, educating health care workers, telephone counseling, and informational approaches were effective. Further, restricting smoking at the workplace was an effective organizational intervention. Finally, policy interventions that promoted smoke-free environments, increased tobacco prices, and enacted tobacco control policies proved effective in lowering smoking rates.

A similar approach to smoking cessation was used to help increase diabetes selfmanagement (Sallis et al., 2008). At the individual level, patients were educated on skills specific to diabetes and strategies for overcoming challenges. One level up, on-going follow-up and support was provided to give patients encouragement. Community resources were also identified that would help patients engage in regular physical activity and adopt a healthy diet. Finally, factors such as stabile quality care, self-management support, and access to resources were addressed at the policy level because researchers understood that policy influences the resources and choices available to people.

\section{Summary}

It is clear that the US has struggled with health disparities for decades, a strain that can be seen more significantly in certain subsets of the population. Research has consistently shown that levels of health and wellness among firefighters are lower than the general population. 
Further, rural regions, such as Appalachia, tend to be plagued with greater health disparities than their urban counterparts. The risks associated with obesity are grave for any person, but because firefighters are responsible for the safety and livelihood of others, the magnitude of their health cannot be understated. Unfortunately, interventions to help firefighters become healthier are still not effective because researchers lack a consistent understanding of factors that influence health behaviors of firefighters. An SEM approach has been useful in understanding health behaviors of different populations. Using this approach, it is important to consider the interaction of each individual with their environment. As such, research must continue exploring the interaction of firefighters in the context of their department, and the broader environment. If firefighters and Appalachian residents are at higher risk for health disparities, does that potentially mean that Appalachian firefighters are at even greater risk? Are the barriers to, and facilitators of, health behavior in this region different compared to other regions? How do the experiences of career and volunteer firefighters differ? These are all questions that research can continue exploring to further guide future interventions. 
Works Cited

Amarasinghe, A., D’Souza, G., Brown, C., Oh, H., \& Borisova, T. (2009). The influence of socioeconomic and environmental determinants on health and obesity: A West Virginia case study. International Journal of Environmental Research and Public Health, 6, 22712287.

Appalachian Regional Commission. (2010). Economic Assessment of Appalachia. An Appalachian Development Initiative Report. Retrieved from http://www.arc.gov/images/newsroom/publications.EconimicAssessmentofAppalachiaJu ne2010.pdf.

Appalachian Research Commission (ARC). (2013, April 16). Retreived from http://www.arc.gov/.

Baur, D. M., Christophi, C. A., Tsismenakis, A. J., Jahnke, S. A., \& Kales, S. N. (2012). Weightperception in male career firefighters and its association with cardiovascular risk factors. BMC Public Health, 12(480), 1-8.

Blake, K. B., Shankar, A., Madhavan, S., \& Ducatman, A. (2010). Associations among cardiometabolic risk factor clustering, weight status, and cardiovascular disease in an Appalachian population. The Journal of Clinical Hypertension, 12(12), 964-972. doi: 10.1111/j.1751-7141.2010.00078.x

Bronfenbrenner, U. (1977). Toward an experimental ecology of human development. American Psychologist, 32, 513-531.

Byczek, L., Walton, S. M., Conrad, K. M., Reichelt, P. A., \& Samo, D. G. (2004). Cardiovascular risks in firefighers: Implications for occupational health nurse practice. American Association of Occupational Health Nurses, 52(2), 66-76. 
Centers for Disease Control and Prevention (CDC). (2006). Fatalities among volunteer and career firefighters - United States, 1994-2004. Morbidity and Mortality Weekly Report (MMWR), 55(16), 453-455.

Centers for Disease Control and Prevention (CDC). (2007). Estimated county-level prevalence of diabetes and obesity - United States, 2007. Morbidity and Mortality Weekly Report, $58(45), 1259-1263$.

Centers for Disease Control and Prevention (CDC). (2009). Estimated county-level prevalence of diabetes and obesity - United States, 2007. Morbidity and Mortality Weekly Report, $58(45), 1259-1263$.

Centers for Disease Control and Prevention (CDC). (2012). Prevalence of Self-Reported Obesity Among U.S. Adults. Retrieved from http://www.cdc.gov/obesity/data/adult.html.

Dienstbier, R. A. (1989). Arousal and physiological toughness: Implications for mental and physical health. Psychological Bulletin, 96, 84-100.

Dienstbier, R. A. (1991). Behavioral correlates of sympathoadrenal reactivity: The toughness model. Medicine \& Science in Sports \& Exercise, 23, 846-852.

Durand, G., Tsismenakis, A. J., Jahnke, S. A., Baur, D. M., Christophi, C. A., \& Kales, S. N. (2011). Firefighters' physical activity: Relation to fitness and cardiovascular disease risk. Medicine \& Science in Sports \& Exercise, 43(9), 1752-1759.

Eberhardt, M. S., \& Pamuk, E. R. (2004). The importance of place of residence: Examining health in rural and nonrural areas. American Journal of Public Health, 94(10), 16821686.

Elliot, D. L., Goldberg, L., Duncan, T., Kuehl, K. S., Moe, E., Breger, R. K., . . Stevens, V. J. (2004). The PHLAME Firefighters' study: Feasibility and findings. American Journal of 
Health Behavior, 28(1), 13-23.

Elliot, D. L., Goldberg, L., Kuehl, K. S., Moe, E. L., Breger, R. K., \& Pickering, M. A. (2007). The PHLAME (Promoting healthy lifestyles: Alternative models' effects) Firefighter study: Outcomes of two models of behavior change. Journal of Occupational and Environmental Medicine, 49, 204-213.

Fahy, R. F., LeBlanc, P. R., \& Molis, J. L. (2012). Firefigher fatalities in the United States 2011. Fire Analysis and Research Division. Quincy, MA: National Fire Protection Association. Retrieved January 2013, from http://www.nfpa.org

Frattaroli, S., Pollack, K. M., Bailey, M., Schafer, H., Cheskin, L. J., \& Holtgrave, D. R. (2012). Working inside the firehouse: Developing a participant-driven intervention to enhance health-promoting behaviors. Health Promotion Practice, 22, 1-8.

Geibe, J. R., Holder, J., Peeples, L., Kinney, A. M., Burress, J. W., \& Kales, S. N. (2008). Predictors of on-duty coronary events in male firefighters in the United States. The American Journal of Cardiology, 101(5), 586-589.

Glasser, M., Holt, N., Hall, K., Mueller, B., Norem, J., Pickering, J., Brown, K., \& Peters, K. (2003). Meeting the needs of rural populations through interdisciplinary partnerships. Family and Community Health, 26(3), 230-245.

Golden, S. D., \& Earp, J. A. L. (2012). Social ecological approaches to individuals and their contexts: Twenty years of health education and behavior health promotion interventions. Health Education \& Behavior, 39(3), 364-372.

Guidotti, T. L. (1992). Human factors in firefighting: Ergonomic-, cardiopulmonary-, and psychogenic stress-related issues. International Archives of Occupational and Environmental Health, 64, 1-12. 
Harris, C. V., Bradlyn, A. S., Tompkins, N. O., Purkey, M. B., Kennedy, K. A., \& Kelley, G. A. (2010). Evaluating the West Virginia Healthy Lifestyles Act: Methods and procedures. Journal of Physical Activity and Health, 7(1), S31-S39.

Jahnke, S. A., Poston, W. C., Haddock, C. K., Jitnarin, N., Hyder, M. L., \& Horvath, C. (2012). The health of women in the US fire service. BMC Women's Health, 12(39), 1-20.

Jahnke, S. A., Poston, W. S., Jitnarin, N., \& Haddock, K. (2012). Health concerns of the U.S. Fire Service: Perspectives from the firehouse. American Journal of Health Promotion, 27(2), 111-118.

Kales, S. N., Aldrich, J. M., Polyhronopoulos, G. N., Leitao, E. O., Artzerounian, D., Gassert, T. H., . . Christiani, D. C. (1999). Correlates of fitness for duty in hazardous materials firefighters. American Journal of Industrial Medicine, 36, 618-629.

Kales, S. N., Soteriades, E. S., Christophi, C. A., \& Christiani, D. C. (2007). Emergency duties and deaths from heart disease among firefighters in the United States. The New England Journal of Medicine, 356(12), 1207-1215.

Kruger, T. M., Swanson, M., Davis, R. E., Wright, S., Dollarhide, K., \& Schoenberg, N. E. (2012). Formative research conducted in rural Appalachia to inform a community physical activity intervention. American Journal of Health Promotion, 26(3), 143-151.

Lox, C., Martin Ginis, K., \& Petruzzello, S. (2010). The Psychology of Exercise: Integrating Theory and Practice ( $3^{\text {rd }}$ ed.). Scottsdale, AZ: Holcomb Hathaway Publishers.

McLeroy, K. R., Bibeau, D., Steckler, A., \& Glanz, K. (1988). An ecological perspective on health promotion programs. Health Education Quarterly, 15(4), 351-377.

Miller, W. R. (1985). Motivation for treatment: A review with special emphasis on alcoholism. Psychological Bulletin, 98, 84-107. 
Moe, E. L., Elliot, D. L., Goldberg, L., Kuehl, K. S., Stevens, V. J., Breger, R. K., . . Dolen, S. (2002). Promoting healthy lifestyles: Alternative models' effects (PHLAME). Health Education Research Theory \& Practice, 17(5), 586-596.

National Fire Protection Agency (NFPA). (2013, February 15). Retrieved from http://www.nfpa.org/index.asp?cookie_test=1.

National Volunteer Fire Council (NVFC). (2013, April). Heart-Healthy Firefighter Program. Retrieved from http://www.nvfc.org/programs/heart-healthy-firefighter-program.

Parks, S. E., Housemann, R. A., \& Brownson, R. C. (2003). Differential correlates of physical activity in urban and rural adults of various socioeconnomic backgrounds in the United States. Journal of Epidemiological Community Health, 57, 29-35.

Peate, W., Lundergan, L., \& Johnson, J. J. (2002). Fitness self-perception and VO2max in firefighters. Journal of Occupational and Environmental Medicine, 44(6), 546-550.

Poston, W. S., Haddock, K., Jahnke, S. A., Jitnarin, N., Tuley, B. C., \& Kales, S. N. (2011). The prevalence of overweight, obesity, and substandard fitness in a population-based firefighter cohort. Journal of Occupational and Environmental Medicine, 53(3), 266-273.

Poston, W. S., Jitnarin, N., Haddock, C. K., Jahnke, S. A., \& Tuley, B. C. (2011). Obesity and injury-related absenteeism in a population-based firefighter cohort. Obesity, 19(10), 2076-2081.

Prochaska, J. O., \& DiClemente, C. C. (1992). Stages of change in the modification of problem behaviors. In Hersen, M., Eisler, R. M., and Miller, P. M. (eds), Progress in Behavior Modification. Pacific Grove, CA: Brooks/Cole, p. 28.

Ranby, K. W., MacKinnon, D. P., Fairchild, A. J., Elliot, D. L., Kuehl, K. S., \& Goldberg, L. (2011). The PHLAME (Promoting healthy lifestyles: Alternative models' effects) 
Firefighter study: Testing mediating mechanisms. Journal of Occupational Health Psychology, 16(4), 501-513.

Rhea, M. R., Alvar, B. A., \& Gray, R. (2004). Physical fitness and job performance of firefighters. Journal of Strength and Conditioning Research, 18(2), 348-352.

Roberts, M. A., O'Dea, J., Boyce, A., \& Mannix, E. T. (2002). Fitness levels of firefighter recruits before and after a supervised exercise training program. Journal of Strength and Conditioning Research, 16(2), 271-277.

Rye, J. A., Rye, S. L., Tessaro, I., \& Coffindaffer, J. (2009). Perceived barriers to physical activity according to stage of change and body mass index in the West Virginia WISEWOMAN population. Women's Health Issues, 19, 126-134.

Saelens, B. E., Sallis, J. F., \& Frank, L. D. (2003). Environmental correlates of walking and cycling: Findings from the transportation, urban design, and planning literatures. Annals of Behavioral Medicine, 25, 80-91.

Sallis, J. F., \& Owen, N. (2002). Ecological models of health behavior. In K. Glanz, B. K. Rimer, \& F. M. Lewis (Eds.), Health behavior and health education: Theory, research, and practice $\left(3^{\text {rd }}\right.$ ed.) (pp. 462-484). San Francisco, CA: Jossey-Bass.

Sallis, J. F., Owen, N., \& Fisher, E. B. (2008). Ecological models of health behavior. In K. Glanz, B. K. Rimer, \& K. Viswanath (Eds.), Health behavior and health education: Theory, research, and practice (4th ed.) (pp. 465-486). San Francisco, CA: Jossey-Bass.

Scanlon, P., \& Ablah, E. (2008). Self-reported cardiac risks and interest in risk modification among firefighters: A survey-based study. Journal of American Osteopathic Association, 108(12), 694-698.

Serrano, E., Leiferman, J., \& Dauber, S. (2007). Self-efficacy and health behaviors toward the 
prevention of diabetes among high risk individuals living in Appalachia. Journal of Community Health, 32(2), 121-133. doi: 10.1007/s10900-006-9034-4

Short, V. L., Oza-Frank, R., \& Conrey, E. J. (2012). Preconception health indicators: A comparison between non-Appalachian and Appalachian women. Maternal and Child Health Journal, 16(2), 238-249.

Soteriades, E. S., Hauser, R., Kawachi, I., Liarokapis, D., Christiani, D. C., \& Kales, S. N. (2005). Obesity and cardiovascular disease risk factors in firefighters: A prospective cohort study. Obesity Research, 13(10), 1756-1762.

Soteriades, E. S., Targino, M. C., Talias, M. A., Hauser, R., Kawachi, I., Christiani, D. C., \& Kales, S. N. (2011). Obesity and risk of LVH and ECG abnormalities in US firefighters. Journal of Occupational and Environmental Medicine, 53(8), 867-871.

Sothmann, M.S., Buckworth, J., Claytor, R. P., Cox, R. H., White-Welkley, J.E., \& Dishman, R. K. (1996). Exercise training and the cross-stressor adaptation hypothesis. Exercise \& Sport Sciences Reviews, 24, 267-287.

Staley, J. A., Weiner, B., \& Linnan, L. (2011). Firefighter fitness, coronary heart disease, and sudden cardiac death risk. American Journal of Health Behavior, 35(5), 603-617.

Stokols, D. (1992). Establishing and maintaining healthy environments: Toward a social ecology of health promotion. American Psychologist, 47(1), 6-22.

Stokols, D. (1996). Translating social ecological theory into guidelines for community health promotion. American Journal of Health Promotion, 10(4), 282-298.

Swank, A. M., Adams, K. J., Barnard, K. L., Berning, J. M., \& Stamford, B. A. (2000). Agerelated aerobic power in volunteer firefighters: A comparative analysis. Journal of Strength and Conditioning Research, 14(2), 170-174. 
Tremblay, M. S., Colley, R. C., Saunders, T. J., Healy, G. N., \& Owen, N. (2010). Physiological and health implications of a sedentary lifestyle. Applied Physiology, Nutrition, and Metabolism, 35, 725-740.

TriData Corporation (2005). The economic consequences of firefighter injuries and their prevention. Final report. Arlington, VA: National Institute of Standards and Technology (NIST). Retrieved December 2012, from http://www.fire.nist.gov/bfrlpubs/NIST_GCR_05_874.pdf

Trost, S. G., Owen, N., Bauman, A. E., Sallis, J. F., \& Brown, W. (2002). Correlates of adults' participation in physical activity: Review and update. Medicine \& Science in Sports \& Exercise, 34(12), 1996-2001.

Wewers, M. R., Kats, M., Paskett, E. D., \& Fickle, D. (2006). Risky bheaviors among Ohio Appalachian adults. Preventive Chronic Disease, 3(4), A127.

Wilcox, S., Castro, C., King, A. C., Housemann, R., \& Brownson, R. C. (2000). Determinants of leisure time physical activity in rural compared with urban older and ethnically diverse women in the United States. Journal of Epidemiological Community Health, 54, 667-672.

Williford, H. N., Duey, W. J., Olson, M. S., Howard, R., \& Wang, N. (1999). Relationship between fire fighting suppression tasks and physical fitness. Ergonomics, 42(9), 11791186.

Zizzi, S., Goodrich, D., Wu, Y., Parker, L., Rye, S., Pawar, V., Mangone, C., \& Tessaro, I. (2006) Correlates of physical activity in a community sample of older adults in Appalachia. Journal of Aging and Physical Activity, 14, 423-438. 


\section{Appendix B}

\section{Consent Form}

\section{Only Minimal Risk Consent Information Form (without HIPAA)}

Principal Investigator
Department
Protocol Number
Study Title
Activity Behaviors
Co-Investigator(s)
Sponsor (if any)

Principal Investigator

Department

Protocol Number

Study Title

Co-Investigator(s)

Contact Persons

In the event you experience any side effects or injury related to this research, you should contact Chelsea Wooding at (304) 293-0867. (After hours contact: Chelsea Wooding at (714) 815-3418). If you have any questions, concerns, or complaints about this research, you can contact Dr. Sam Zizzi at (304) 293-0874.

For information regarding your rights as a research subject, to discuss problems, concerns, or suggestions related to the research, to obtain information or offer input about the research, contact the Office of Research Integrity \& Compliance at (304) 293-7073.

In addition if you would like to discuss problems, concerns, have suggestions related to research, or would like to offer input about the research, contact the Office of Research Integrity and Compliance at 304-293-7073.

\section{Introduction}

You, , have been asked to participate in this research study, which has been explained to you by Chelsea Wooding. This study is being conducted by Chelsea Wooding in the Department of Sport and Exercise Psychology at West Virginia University.

\section{Purpose(s) of the Study}

The purpose of the study is to gain a better understanding of West Virginia firefighters' health behavior. Specifically, the aim is to learn about facilitators of, and barriers to, physical activity and healthy eating, with the hope of informing future research and intervention development. The larger goal is to learn more about how we might be able to help firefighters become healthier, and thus safer on the job. A maximum of 120 firefighters are expected to participate.

\section{Description of Procedures}

This study involves participating in a focus group with your fellow firefighters. Each focus group will take approximately 60 minutes. You will also be asked to fill out a brief demographics questionnaire before the focus group begins. This will take approximately 5-10 minutes. You do not have to answer all of the questions. You will have the opportunity to see the questionnaire before signing the consent form. Your participation in the focus group and completing the questionnaire is completely voluntary. You may choose not to answer any questions that you do not feel comfortable answering and you may discontinue your participation in this focus 
group at any time. Your choice to participate or not will not be reported to any of your supervisors.

Focus groups will be audio recorded. Recordings will be kept on a password protected computer in a locked office until transcriptions are complete, at which point they will be deleted.

After the focus groups, you will be invited to participate in a secondary part of the study. If you chose to participate, you will be asked to take pictures of factors in your daily life that influence your physical activity and nutrition habits, and to send those pictures along with a 1-2 sentence explanation to the lead researcher. If you have a smart phone, pictures can be sent through SMS text message. If you do not have a smart phone, disposable cameras will be provided, along with a padded, self-addressed and stamped envelope for you to return the cameras after one week. If pictures are taken with a disposable camera, you will be asked to keep simple notes (1-2 sentences) about the pictures you took and send those notes back with the camera. Again, your choice to participate or not will not be reported to any of your supervisors.

\section{Discomforts}

There are no known or expected risks from participating in this study, except for the mild frustration associated with answering the questions or taking part in a focus group.

\section{Benefits}

You may not receive any direct benefit from this study. The knowledge gained from this study may eventually benefit others.

\section{Financial Considerations}

There are no special fees for participating in this study, but food will be provided at the end of the focus group.

\section{Confidentiality}

Any information about you that is obtained as a result of your participation in this research will be kept as confidential as legally possible. Your research records and results, just like hospital records, may be subpoenaed by court order or may be inspected by federal regulatory authorities (including the FDA if applicable) without your additional consent.

In addition, there are certain instances where the researcher is legally required to give information to the appropriate authorities. These would include mandatory reporting of infectious diseases, mandatory reporting of information about behavior that is imminently dangerous to your child or to others, such as suicide, child abuse, etc.

Audiotapes will be kept locked up and will be destroyed as soon as possible after the research is finished. In any publications that result from this research, neither your name nor any information from which you might be identified will be published without your consent.

\section{Voluntary Participation}

Participation in this study is voluntary. You are free to withdraw your consent to participate in this study at any time.

Refusal to participate or withdrawal will not affect you and will involve no penalty to you. 
In the event new information becomes available that may affect your willingness to participate in this study, this information will be given to you so that you can make an informed decision about whether or not to continue your participation.

You have been given the opportunity to ask questions about the research, and you have received answers concerning areas you did not understand.

Upon signing this form, you will receive a copy.

I willingly consent to participate in this research.

\section{Signatures}

Signature of Subject

Printed Name

Date

Time

The participant has had the opportunity to have questions addressed. The participant willingly agrees to be in the study.

Signature of Investigator or Co-Investigator

Printed Name

Date

Time 


\section{Appendix C}

\section{Demographic Questionnaire}

Please do NOT write your name on this questionnaire.

1. What is your current age?

2. What is your sex?
a. Male
b. Female

3. What is your current marital status?
a. Married
b. Divorced
c. Widowed
d. Separated
e. Never married
f. A member of an unmarried couple

4. About how much do you weigh without shoes (in pounds)?

5. About how tall are you without shoes?

Feet, Inches

6. Which one of these groups would you say best represents your race?
a. White
b. Black/African American
c. American Indian/Alaska Native
d. Asian
e. Hispanic/Latino/Spanish
f. Pacific Islander
g. Other,

7. What is your annual household income from all sources?
a. Less than $\$ 25,000$
b. $\$ 25,000$ to $\$ 34,999$
c. $\$ 35,000$ to $\$ 49,999$
d. $\$ 50,000$ to $\$ 74,999$
e. $\$ 75,000$ to $\$ 99,999$
f. $\$ 100,000$ or more

8. What is the highest grade or year of school you completed?
a. Grades 9 through 11 (some high school)
b. Grade 12 or GED (high school graduate)
c. College 1 year to 3 years (some college or technical school)
d. College 4 years or more (college graduate) 
e. Graduate school (Master's or beyond)

9. How long have you served as a firefighter (years)?

10. How long have you served as a firefighter in WV (years)?

11. How long have you served at your current fire department (years)?

12. Have you ever been told by a doctor, nurse, or other health professional that you have any of the following (please circle the appropriate answer):

\begin{tabular}{|c|c|c|c|c|c|}
\hline High Blood Pressure & Yes & No & Don't know & $\begin{array}{c}\text { Borderline or } \\
\text { pre- } \\
\text { hypertensive }\end{array}$ & $\begin{array}{c}\text { Yes, but female } \\
\text { told only during } \\
\text { pregnancy }\end{array}$ \\
\hline High Cholesterol & Yes & No & Don't know & & \\
\hline $\begin{array}{c}\text { A Heart Attack } \\
\text { (Myocardial } \\
\text { Infarction) }\end{array}$ & Yes & No & Don't know & & \\
\hline $\begin{array}{c}\text { Angina or Coronary } \\
\text { Heart Disease }\end{array}$ & Yes & No & Don't know & & \\
\hline Stroke & Yes & No & Don't know & & \\
\hline $\begin{array}{c}\text { Cardio-Pulmonary } \\
\text { Disease (CPD) }\end{array}$ & Yes & No & Don't know & & \\
\hline Diabetes & Yes & No & Don't know & & \\
\hline
\end{tabular}

Thank you for your time. 


\section{Appendix D}

\section{Focus Group Protocol}

\section{Introduction (10 minutes)}

- Hello and welcome to our session. Thank you for taking time to join us today.

- (Introduction of staff)

- (Describe what a focus group is)

- We will be talking with you today about health behaviors of firefighters.

$\circ$ Learn from you about your thoughts on this.

- Please feel free to share your ideas and opinions even if they are different from others.

- No right or wrong answers

- Get as many different points of view as we can

$\circ$ All views and ideas are important

- Anything we say here today is confidential. Individual names or answers will not be shared with anyone. Only a summary of all groups we do will be compiled.

- We also hope you do not share with others anyone's individual answers from the group today.

- The session should last about an hour.

- Before we begin we need to have you sign a consent form. (Pass out consent forms for signature)

- We will be taping this discussion to make sure we don't miss any of your comments.

○ Notes are not as complete as when we tape record the discussion.

- Try to speak up so the tape recorder picks up your answers.

- Since this is a group discussion you do not have to wait for me to call on you to speak.

- Please try to speak one at a time.

\section{Opener/Prompt}

- "What impacts your health as a firefighter in West Virginia?"

\section{Potential Follow-Up Questions (if needed)}

- What barriers keep you from eating healthier?

- What barriers keep you from being physically active?

- What supports healthy eating?

- What supports being physically active?

- What kind of changes could be made to help firefighters become healthier?

- What are some reasons that firefighters become overweight or obese?

- What are some concerns or problems that overweight or obese firefighters have?

- Are there any questions you have? 


\section{Appendix E}

\section{Extended Results}

\section{Participant Demographics}

A total of eight focus groups were conducted, four in volunteer departments and four in career departments. Two of the career departments recruited were classified as combination departments; one focus group at a combination department had only career firefighters present (n $=4$ ), and the other focus group had one career and two volunteer firefighters present. One focus group with each type of department was done in each of the four different RUCA code levels (i.e., urban, large rural, small rural, isolated rural). A total of 58 participants contributed to the eight focus groups; 27 career firefighters and 31 volunteer firefighters. The average age of participants was 38.79 years old; the youngest participant was 19 years old and the oldest was 66 years old. Four out of the 58 participants were female. Overall, $69 \%$ of the participants were married, and all 58 participants were white. About $26 \%$ of participants reported their annual income as between $\$ 35,000$ and $\$ 49,999$, about the median household income in West Virginia from 2007 to 2011 (US Census Bureau, 2012). However, 48.3\% of participants reported making $\$ 50,000$ or more per year. With regards to education, $36.2 \%$ reported being a high school graduate, and $43.1 \%$ reported completing some college or technical school. Overall, $79.3 \%$ of participants held a high school diploma, a percent close to the US Census Bureau data from the state of West Virginia. Finally, when asked about seven different health risks from the BRFSS, $15.5 \%$ of participants have been told by a doctor that they have high blood pressure, $19.0 \%$ have high cholesterol, and $8.6 \%$ have diabetes. Only two participants reported having a stroke in the past, and only one participant reported having COPD, coronary heart disease, and having a heart attack in the past. 
On average, participants had been firefighting for just under 16 years, had been firefighting in West Virginia for about 15 years, and had been firefighting at their current department for 14 years; the shortest time a participant had been firefighting was just under a year, and the longest time was 45 years. There was no significant difference between career and volunteer firefighters in the total amount of years served firefighting, firefighting in West Virginia, or firefighting at their current department; nor was there any difference in the years served based on setting (i.e., urban, large rural, small rural, and isolated rural).

The average BMI for the participants was 28.78, with a low of 20.4 and a high of 44.9 . These numbers show that, on average, the group of participants was overweight. Considering that many people under-estimate weight when asked to self-report, the group of participants could be close to qualifying as obese. Again, there were no significant differences in BMI between career and volunteer firefighters, but the BMI of volunteers was slightly higher than careers at 29.47 versus 27.99 respectively. There also were no significant differences in BMI between different settings, but those participants in isolated rural settings had an average BMI slightly higher than other settings (30.63 for isolated rural, 28.85 for small rural, 28.24 for large rural, and 28.01 for urban settings).

\section{Focus Groups}

From the focus group data, eight factors were identified as impacting the health of West Virginia firefighters: job related concerns, general factors impacting health, time, nutrition, physical activity, motivation, sleep, and stress.

Job related health concerns. All eight of the focus groups talked about aspects of firefighting that effect their health. Inhalants, such as chemicals, smoke, plastic, carcinogens, diesel smoke, and fumes, were discussed the most; specifically, five of the eight focus groups 
discussed the dangers of different chemicals to which firefighters are exposed on the job. Along with inhalants, the task of firefighting poses health concerns, such as the heat of a fire, or the physicality required to perform the job; two focus groups discussed each of those concerns. Additionally, two focus groups also talked about the weight of the equipment and gear, and the potential injury that can occur because of that added weight. Two focus groups also mentioned the dangers of dehydration on the job, and the importance of drinking water while fighting a fire. Interacting with others in the course of firefighting was also reported as a concern related to firefighting. Facing traffic when driving to a call, and travelling to a call in general were each discussed by two focus groups as factors that impact the health of firefighters in the course of performing, or attempting to perform, their job. Illness, such as the spread of illness when interacting with people on a call, or family illness being transferred to the firefighters, and age were additional factors discussed.

General factors impacting health. Specific concerns related to nutrition, physical activity, stress, and sleep were discussed in each focus group. However, participants also talked about generic factors unrelated to a specific aspect of health that influence their health behaviors; those factors were grouped here as general factors that influence health, general barriers to health behaviors, and general facilitators of health behaviors.

General. In general, the factor discussed the most was internal factors that affect health. More specifically, five of the eight focus groups discussed the power of motivation and how it can either help or hurt a person's health. One participant explained the importance of motivation by simply saying, "It's all self-motivation. It is, it's a lot." Different focus groups echoed that sentiment, explaining that motivation is often the most important factor related to health. Other internal factors that firefighters reported affect their health included body type, age, personal 
pride in their department, and time management.

Along with internal factors, external influences were also identified by the participants. External factors discussed in focus groups included the influence of legislation, lack of social support among family members, the physical demands of the job, and having few or no days off from firefighting. The final general influence mentioned was experience, specifically how a firefighter's perceptions of health and its importance change with experience, and a firefighter's work background.

Barriers. The main sources of barriers that generally effect the health of firefighters include personal barriers, job barriers, environmental barriers, system barriers, barriers related to other people, and barriers related to choices that are made. When discussing personal barriers, half of the focus groups talked about lack of time and age as barriers to healthy living. Because of the time demands related to firefighting, working other jobs, and having a family, participants reported having very little time, if any, to focus on their health and wellbeing. Further, firefighters felt that as they got older, it became more challenging to stay healthy and active. One participant said, "As we get older, we kinda cut back on a lot of things too, and um, and then to build muscles you have to cut back on some of that [eating], so we just hope to stay healthy." Lack of motivation was another personal barrier discussed by three of the focus groups. Participants noted that without motivation, no one will make any changes to become healthier. One participant explained, "I guess at the bottom of it all is motivation, both for eating right and to exercise. And like [participant] said, we all have a lot of things to do, and we don't want to give up those few spare minutes you have to do that." Two of the eight focus groups also brought up unhealthy habits and previous health conditions as well. Participants felt that their everyday habits were unhealthy, which promoted further unhealthy behavior. In addition, if a 
firefighter had a previous health condition, such as a heart problem or illness, they perceived that condition to be a barrier to living a healthier lifestyle. Other personal barriers discussed by only one focus group each included lack of knowledge, sleep, everyday life, lack of exercise, perceptions of being in good health, inactivity, and previous injury.

Along with personal barriers, participants discussed the negative influence of barriers from their work as a firefighter. The most commonly discussed barrier here, brought up by five of the eight focus groups, was stress, including mental and emotional stress. Though some participants were unable to articulate exactly how the stress impacted them negatively, all felt that stress was a barrier to their health, and many believed stress to be the number one reason for health problems among firefighters. Another commonly discussed job barrier, mentioned in half of the focus groups, was the uncertain schedule of firefighters. Both career and volunteer firefighters explained that they can have the best of intentions, attempting to plan physical activity or healthy cooking in their day, but because they could be called out at any given moment, they are often interrupted during their attempts at healthy living, or do not even try to be healthy. One participant shared, "We joke and say it's like war, you have to eat when you can and sleep when you can because you don't know what's going to happen when." Other barriers from work as a firefighter included perceptions of ability to successfully perform the job in current health, weight gain from the job, injury on the job, adrenaline, and departmental chores. Another category of barriers that arose from the focus groups was that of environmental barriers. Three focus groups talked about the role of society; that the health and obesity problems within the fire department are a microcosm of the problems in the larger society today. Weather was another barrier brought up by three focus groups. Unfortunately, the winter was quite harsh, which participants' felt limited the opportunities for healthy behaviors such as 
physical activity and access to healthier foods. Other environmental barriers included an unhealthy home environment, and unhealthy work environment, the economy, a fast-paced society, reliance on machines, and cost.

Participants also mentioned certain system barriers in existence that negatively impact their health. Three focus groups talked about the need to find a job for everyone in the department, regardless of their health. Because the departments are so short-staffed, departments are not able to turn people away because of health concerns. Instead, the firefighters work to find a job for any active firefighter in their department. One participant explained the experience of their chief,

The chief here told me one time that like he was hinting on you know, he may not be able to tag along, but he might be able to run an engine or a pumper or a tanker. I mean, there's a job for everybody. And, traffic detail. Anything, roll up hoses, wash hoses, I mean, there's lots of stuff that you know, everybody can do.

Other system barriers that effect the health of firefighters included budget priorities, the size of the department, lack of system progress, no physical fitness requirements in West Virginia, and taking advantage of previous health incentives.

Although less frequent, participants brought up numerous choices they make that create barriers to healthy living. In general, two focus groups identified their lifestyle as a barrier to health and wellness. Other choices were drinking soda, eating too much, eating unhealthy options, ignoring health concerns, and smoking.

Finally, participants also discussed the role of other people as a barrier to their health. One focus group each mentioned family sickness, lack of social support, unhealthy role modeling, and negative social pressure as ways that other people in their lives, both in and out of 
the department, negatively impact their health.

Facilitators. Though the participants mentioned a significant number of barriers to health, they also identified multiple factors that encourage healthy living. The first category of facilitators of health for firefighters was awareness. Six of the eight focus groups identified heart attack as the number one cause of on-duty death for firefighters. Participants reported that their awareness of the on-the-job risk they face encourages them to lead healthier lifestyles. Awareness of other risks, such as cancer and short life expectancy, also act as facilitators. Three of the focus groups also discussed the importance of each firefighter knowing their limits, and working within those limitations. Without awareness of those limits, firefighters could be in much more danger than they already are. One volunteer participant explained, "Whether you're overweight, you're underweight, or whatever you are, you know your limits and you have to, you know, you know your body and what you can do and what you can't." Similarly, a career participant explained, "We all know our personal limitations pretty well and keep those in check." The last factor related to awareness brought up by one focus group was awareness of what can be controlled.

Personal factors and personal choices also serve as facilitators to health behavior. Two focus groups mentioned that they had started drinking more water and less soda. For them, this was a positive change that continues to facilitate health. Personal motivation, staying active, maintaining healthy eating habits, and trying to stay healthy were other personal factors that facilitated health behaviors for the participants.

While the system was a source of barriers to health, it also provides some facilitators as well. Two focus groups discussed a safe work environment as a facilitator to their health. One focus group pointed to the health improvements of their department as a facilitator of continued 
health behavior. Focus on prevention, better equipment, and preventative health measures like vaccines were other aspects of the system of firefighting that participant's felt positively influenced their health.

Along with awareness, personal factors, and the system, job and departmental requirements can also positively impact health. One focus group mentioned that the sobriety requirements for firefighting encouraged them to significantly lessen their alcohol consumption. Another focus group discussed the limited staff at their department as a facilitator to healthy living because, if they are no longer able to actively serve due to health problems, the department would suffer as they have few people that could act as a replacement. Finally, one focus group identified training as a protective factor while firefighting, encouraging future healthy behavior.

The final facilitator to health in general is the influence of others. After identifying unhealthy role modeling as a barrier to healthy living, the same focus group mentioned that healthy role modeling can be equally as impactful to encourage healthy behavior. Another focus group discussed the younger generation entering the fire service as a facilitator of health behavior because they appreciate healthy behavior, like physical activity, more than some of the older generations, and bring with them a healthier mindset.

Time. The influence of time was mentioned in regards to a number of different behaviors related to health. However, all eight of the focus groups discussed the time demands that come with trying to balance working, firefighting, and having a family. Volunteer departments discussed time demands as they related to working a full-time job, then serving at the fire house in between normal work hours, while spending time with family, and many trying to raise children. One volunteer explained his experience,

You're trying to work, you know, work the workload that you got at work, plus you're a 
volunteer in the community, then you got your wife and your kids, and if they're in sports. I mean, I volunteer at the baseball fields too, I coach two baseball teams and you just, you just get wrapped up in everything and you try to, you know, be there and do everything you can for everybody, and sometimes it works, and sometimes it don't. Career departments discussed the same concerns, including the time constraints related to working a full-time job as a firefighter, and also working at least one other job, if not two. One career firefighter explained, "Most of us have a second job, so as soon as we get out of here at eight o'clock, you know, they're going to another eight hour job." With all of the demands on their time between firefighting, working, and maintaining a personal life, firefighters discussed the near impossibility of finding time for health behaviors such as cooking healthy meals or engaging in physical activity. Along with the work, firefighting, life balance, two other time concerns, mentioned by one focus group each, were also discussed: lack of time and the uncertainty of schedule.

Nutrition. When discussing factors that influence their health, one major contributor was nutrition. Participants spoke about barriers to healthier eating, facilitators to healthier eating, and general factors that influence the type of foods they consume.

Barriers. Perhaps the most powerful barrier discussed among the focus groups was that of access and availability. Half of the focus groups discussed the ease of unhealthy food, and when considering the time constraints within which they are forced to work, easy food tends to be the food they eat. As one participant said,

Sometimes I'll come home and get in bed at three in the morning and I have to get up at six the next morning. It's a lot easier to throw something in the microwave than it is to cook something that would be a lot healthier for you. 
Further, three focus groups mentioned the availability of, and the ease of access to, unhealthy options. Once again, because their schedules are so sporadic, having more access to unhealthy foods makes them easier to eat, despite desires to eat healthier. Another barrier related to access and availability discussed by three focus groups was the cost of healthy foods compared to the cost of healthy foods. Though many participants expressed an understanding of the importance of healthy eating, many discussed the fact that healthier food is more expensive, eliminating the choice of having healthy options around. One participant explained his frustration when shopping, "You can go down there to the store, you can get a two-liter bottle of Coke for 99 cents. You can get a 20 -ounce bottle for $\$ 1.29$. Or a bottle of water's \$1.99." Another participant expressed the same frustration when eating out, "I've tried it, eating healthy is more expensive. I mean, where’s Subway’s dollar menu? You know, you can’t get full on $\$ 4.00$. I can't get full on $\$ 4.00$ at Subway." Fast food was also discussed as a barrier to healthy eating in relation to access and availability. Focus groups discussed the availability of fast food, the ease of fast food, and eating out in general as factors that encourage unhealthy eating habits rather than facilitating healthy eating. Finally, two focus groups talked about a lack of access to grocery stores, which limits their ability to keep healthy options at home or the fire department. Along with access and availability, another popular barrier discussed by six of the eight focus groups was lack of time. When considering the demands placed on firefighters, between working, volunteering, firefighting, caring for family, training, and other responsibilities, firefighters feel that they simply do not have the time to eat healthier. One career participant explained, “Sometimes you just don't get time to bring food in and cook, you just have to eat when you're out, you don't have a choice. It's the only time you get a chance." Another volunteer participant echoed the same struggles, 
You know, always being on the go, it's not too easy to, especially volunteer wise again, you know, you're working a full-time job, you're doing a, you know, you're not always eating the best. You know, you can't always sit down to a full, four-course meal and you know, enjoy it.

The perceived lack of time in the schedules of both volunteer and career firefighters creates a significant barrier to healthier eating.

During the focus groups, participants took responsibility for their unhealthy choices by discussing personal barriers to nutrition. Half of the focus groups blatantly explained that they simply preferred the taste of unhealthy foods compared to healthier foods. One participant honestly said,

For me, it's just my personal habit. If I have my choice between white bread and wheat bread, I'm always gonna take the white bread. Talk about nutrition, it's like sausage or turkey burger, you know. I take the sausage. I just, that's my taste. I like the unhealthy stuff.

In addition, two focus groups explained that their unhealthy eating was a habit. Other personal barriers discussed included boredom, vacation, not caring about nutrition, and lack of results from healthy eating.

Not surprisingly, factors around the fire department, or those related to firefighting in general, can serve as barriers to nutritious eating habits. Four of the focus groups talked about disrupted meals by telling stories about taking the time to cook a healthier meal, but after taking only one or two bites, they were interrupted by an alarm. Unfortunately, by the time they returned to the department, the meal was no longer good, was thrown out, and fast unhealthy options became the replacement meal. One participant shared, 
I don't know how many times I've sat down at dinner and you start to eat and pager goes off and up you're going and go and time you get back it's cold or it's thrown away ... You come back and if it's cold or you're not in the mood to eat because of whatever you went on.

After a certain time, firefighters then become trained out of trying to cook healthy meals because they are so often interrupted. One participant explained, “After so many times of being interrupted you're like, do I really want to start this, because the last two times I've done it [cooked a healthy meal], it gets interrupted." Stress from firefighting was also brought up by two of the focus groups as another barrier to healthier eating. When experiencing the stress innate to their work, firefighters report that they tend to be drawn to unhealthy foods, overeat, or do not eat enough. Another barrier related to the department is cooking for different tastes. Two focus groups discussed the challenge related to cooking for all of the firefighters, and pleasing different palates is significantly easier with unhealthy foods, rather than healthy items. Other barriers stemming from the department included overeating during social meals, other crew eating food, fatigue, unhealthy environments, sleep deprivation, and inconsistency of diet.

People outside of the fire department also present an interesting barrier to firefighters. Two focus groups brought up unhealthy food donations given to the department by members of the community. While they were thankful for the kind donation, they were unable to select a healthy option when members of the community bring them fast food, or home cooked bakery items. Additionally, family members and home cooking can act as barriers to healthy eating. If the firefighter's home environment does not support healthy eating, that creates a major barrier to eating healthy in general, even outside the home.

The final barrier to nutrition was factors in the environment. Two focus groups discussed 
challenges with eating healthy during the winter. For instance, participants shared that in the winter, they find little to do with their time other than sleep and eat because they are limited in available activities. Additionally, when they eat in the winter, they do not choose the healthiest options. Other environmental barriers included a fast-paced society, TV commercials, serving sizes, and lack of daylight hours.

Facilitators. While many of the factors above were discussed as barriers to healthy eating, some similar categories were identified as facilitators to nutrition. The most commonly discussed facilitator of nutritious eating was planning. Part of planning for firefighters involved understanding that what they eat is fuel for their job, a point discussed by three of the focus groups. Without considering what food will give them the necessary energy to perform well if on a call, participants expressed concern that they might run out of energy in the middle of an emergency. One participant explained,

You have to kind of eat and think about the fact that, okay, I'm gonna eat this meal and then maybe in an hour I'm gonna have to work my tail off. So you don't wanna eat crackers and don't necessarily do crackers and tuna to try to stay healthy because you're gonna burn that off in three seconds.

Knowing that they will need an extreme amount of energy if called out to an emergency encourages them to consider eating healthier throughout the day. Other aspects of planning included planning food preparation, and stocking healthy options at the department.

Another category of facilitators to healthy eating is access and availability. Access and availability can prove to be a barrier, but having access to cooking equipment at the department, and an opportunity to cook while working were both identified as factors that encourage more nutritious meals. Further, one focus group mentioned that there is nutrition education available 
to the firefighters, which they find helpful to learn more about how they can eat healthier.

Other, less common, facilitators to healthy eating included personal factors, other people, the job and department, and the environment. Personal factors also serve as encouragement for healthy eating. More specifically, the general health of firefighters, prioritizing healthy eating, and increased energy from eating healthier facilitate eating healthier. While personal factors are important, other people can also play a positive role by providing social support for healthy eating. Within each department, having a larger department and thus more firefighters to share food costs, and the work environment at the department can have a positive impact on nutrition behavior. Finally, the weather, more specifically summer, can encourage healthy eating habits because of the availability of fresh produce and other healthy options.

General influences. Participants often discussed factors impacting their nutrition habits as either helpful or harmful; however, at times they discussed general factors that influence their nutritional choices. These general influences were access and availability, personal factors, and others.

As with barriers and facilitators, access and availability was a common point brought up in the focus groups. Considerations made by participants when choosing foods included the ease of the food being made, the accessibility of different types of food, the speed at which the food can be made, the limited food options available, and cost. Participants also talked about the personal factors that impact their food choices, which included their age and taste preferences. Other people can also impact their choices, through individual differences and family members.

Physical Activity. Similar to nutrition behavior, participants acknowledged the importance of physical activity in their health, and brought up certain barriers of, and facilitators to engaging in physical activity. 
Barriers. The seven different types of barriers discussed in the focus groups were personal factors, job related factors, system factors, the environment, other people, access and availability, and time.

When discussing why they do not engage in consistent physical activity, participants discussed numerous personal factors. Two focus groups talked about being lazy, that they simply do not have the energy to exercise. Two focus groups also discussed their priorities, and explained that other aspects of their life such as work, firefighting, or family take precedence over trying to find time to exercise. One participant elaborated,

I don't want to be off doing something else when I could be going on calls or could be going on runs. Cause I'm still trying to learn this department and everything there is to learn about being a firefighter being a younger firefighter. Um, so I would say that's one reason why I don't exercise regularly.

Age was brought up by two focus groups as well, that as participants got older they felt less motivated to engage in physical activity. Lack of motivation to exercise was also discussed by two focus groups. Other personal barriers discussed included hating running, preference for inactivity, and previous injury.

Certain components of firefighting and working within the department can also serve as barriers to physical activity. Two focus groups mentioned that potential calls prevent them from wanting to exercise, because they do not want to be working out, lacking energy when an emergency call comes in. Similarly, participants in two focus groups discussed workouts being disrupted by calls in the past, which dropped their motivation to continue with physical activity. The uncertainty of their schedule was also talked about by two focus groups; since they are unable to successfully plan their day, they find it challenging to schedule time for consistent 
physical activity. Other firefighting related barriers included perception of ability to successfully perform the job, having recliners at the department, sleep deprivation, and working a sedentary computer job at the department.

Along with firefighting and each department, participants identified barriers within the system as well. Two focus groups talked about the lack of physical requirements among the fire service, which translates to a lack of motivation to maintain a level of physical fitness because there are no ramifications for remaining inactive. Another system concern discussed by two focus groups was budget constraints or budget limitations. Because their budgets are already stretched so thin, they are unable to either purchase exercise equipment, or pay to maintain, fix, or upgrade the equipment they already have. Other system barriers to physical activity included training time requirements, lack of incentive, and the old school mentality of the fire service.

Beyond the system of the fire service, the environment also serves to negatively impact physical activity among firefighters. Five of the eight focus groups discussed weather, specifically winter weather, as a barrier to engaging in physical activity. One participant simply stated, "Frankly, when it gets cold, I just don't wanna move." Though many career departments have access to equipment at their department, participants still discussed a desire to be active outside, which they do not see as an option during the winter. One career participant said, "This is the biggest station we have, and you feel like a rat in a cage [in winter] because it's just, you know, you can't get out." Volunteer participants also expressed limited physical activity in the winter, as one firefighter explained,

Winter's a tough time here too, because you can't run a lot of times because of ice, if I did run. But I mean there's a lot of stuff you can't do outside because of the weather, and I think we do a lot better here in the spring, in the summer, and the fall. You know, 
there's like three months here where we sometimes have trouble doing anything but shoveling the driveway, and I do that with a machine.

Further, firefighters' preference in the winter is to be sedentary; participants mentioned a pull towards "hibernating" rather than being active once it becomes cold. The other environmental barrier discussed was lack of daylight hours.

Along with the individual and the environment, other people can also act as a barrier to physical activity. Not having a workout partner was a barrier discussed by two focus groups. While participants expressed enjoying being active with others, not having someone to work out with them lessened their motivation to begin, or continue, engaging in physical activity. Other ways that people act as a barrier to physical activity included lack of leadership support and family.

Though not as significant as in the nutrition discussion, access and availability in relation to physical activity was another barrier commonly discussed, mainly among volunteer firefighters. Three focus groups talked about the lack of access to workout equipment as a major barrier for them. One volunteer explained that activity for smaller departments might be more challenging than for larger departments because of the lack of access to equipment,

There isn't a whole lot we can do here because we don't have the weight benches and all that stuff, you know, like some of the bigger departments. They have a lot more room than we do. This is an old building, and we just got the more or less, the basic stuff. But some of these larger departments they have a workout bench and you know, stuff, and they can all hang out down there and do their thing and, but we're not blessed with that. Firefighters that did not have access to equipment felt they would use it if it was made available, but for many departments that was not currently an option. Other access and availability barriers 
included lack of space, wanting better equipment, cost of equipment, lack of exercise education, and cost of available programs.

The final barrier discussed among the focus groups was the impact of time. Two focus groups explained that a lack of time to exercise significantly limits their ability to engage in physical activity. Other time barriers included needing relaxation time, and balancing work, firefighting, and life.

Facilitators. Six of the seven categories of barriers were also identified as facilitators to behavior: personal factors, other people, access and availability, time, environment, and department.

Personal facilitators of physical activity were the most commonly discussed in the focus groups. Two groups talked about physical activity through hobbies, such as basketball and softball. Similarly, three focus groups mentioned the importance of engaging in exercise through preferred activities, rather than forced or regimented exercise programs. One volunteer firefighter explained that she and some other firefighters had “been doing Insanity and runnin' on a treadmill, Bowflex, and that's about it." Other personal factors that can facilitate physical activity included priorities, previous experience with physical activity, current weight, knowledge of the benefits of physical activity, feeling good after exercise, guilt after missing exercise, increased energy from exercise, and unhealthy eating as a reward.

The effect of other people was once again discussed as positively impacting physical activity. Three focus groups mentioned the importance of social support with regards to physical activity. One participant explained the motivation of having someone there to support the workout, "Exercising by yourself is, there's no motivation there. But when you partner up with somebody, you have all the motivation you need right there cause the one pushes the other." 
Other ways in which people can facilitate physical activity included equipment donations and encouragement.

While lack of access to, and availability of, equipment can be a barrier, having access to such equipment can serve as a facilitator to physical activity. Two focus groups discussed that having exercise equipment available at their department made engaging in physical activity that much easier. One career firefighter explained, "I mean, we're lucky here, we have pretty much weight rooms in every station. I think there's a lot of departments out there that probably doesn't have that." Another career participant in the same focus group elaborated,

I mean, us, it's pretty handy because we have them [weight rooms] at the stations. Now if we had to send our guys to the gym, to say Planet Fitness there on south side, that might create big problems, you know what I mean. Might be harder.

Outside of the department, two focus groups also explained that firefighters are often provided membership discounts at local gyms. Therefore, even if the equipment is not available at the department, firefighters can use local gyms to be active. Other access and availability facilitators included group opportunities for physical activity and opportunity for exercise.

The last three factors, environment, time, and the department, were not discussed as frequently, but still can positively influence physical activity behavior. Two focus groups mentioned that weather, specifically warmer weather in the spring and summer, encourages more activity because firefighters can be active outside. In relation to time, one focus group admitted that there is a certainty to the schedule that allows for scheduled physical activity. Another focus group mentioned that making and prioritizing time for physical activity encourages being active. Finally, one focus group talked about required physical activity time at their department, which encouraged the firefighters there to engage in activity throughout the week. 
Motivation. Participants often spoke of the importance of motivation in leading healthy or unhealthy lives. While motivation was commonly discussed in how it impacts nutrition or physical activity, participants also took time to discuss different factors that impact their motivation overall.

Barriers. Firefighters discussed barriers that fell into one of four categories of barriers to motivation: personal barriers, others as a barrier, firefighting, and the system.

Again, one of the most commonly discussed barriers to motivation were personal factors. Three focus groups discussed lacking personal motivation to make healthy lifestyle changes. One participant admitted, "They all [doctors] want you to exercise and lose weight, and it all sounds good, but it, at the end in reality, it's not something that $99.9 \%$ of us wants to do." Additionally, two focus groups talked about knowing they should make changes, and at times wanting to change, but then never following through with those intentions. One volunteer firefighter mentioned, "I know for me, I mean trying to do it is one thing, actually doing it is another." Finally, one focus group mentioned age as a barrier to motivation; as one gets older, they no longer have the energy to put towards health behaviors.

Not surprisingly, other people, both in and out of the fire service, can also influence a firefighter's motivation. One focus group talked about the lack of social support among firefighters to lead a healthier lifestyle. Similarly, one focus group discussed the unhealthy role modeling that can exist within a department. As one firefighter makes an unhealthy decision, the firefighter next to him is tempted to do the same, even if he intends to make a healthier choice. Finally, participants in one focus group expressed a mistrust of doctors, which motivated them to ignore health advice they might receive.

Similar to the effect of others, the system surrounding firefighting can inhibit motivation. 
Lack of support for health behaviors within the fire service was one such barrier discussed in one focus group. One focus group identified the lack of physical fitness requirements as a motivation barrier; because there is no requirement from the service, firefighters lack motivation to stay healthy. Likewise, a lack of incentive from the fire service for healthy behavior also stifles motivation. Participants in one focus group explained that their paycheck does not change if they are healthy or unhealthy, so there is no motivation to become, or stay, healthy.

The final motivation barriers are those related to the job of firefighting. First, participants in one focus group did not perceive a connection between firefighting and nutrition; therefore, there were not motivated to eat healthier, because they did not feel the two were related. Additionally, a lack of motivation stemmed from the job of firefighting itself for one volunteer focus group. One volunteer firefighter felt that motivation to stay healthy for career firefighters comes from getting paid; volunteer firefighters, however, lack the same motivation because they are not paid for their services. Lastly, one focus group mentioned that not fighting many fires can decrease motivation. Since firefighters can go an extended period of time between fire calls, it is easy to forget the amount of fitness and stamina required to successfully perform the job. Consequently, the motivation to make healthy decisions in the day-to-day is lost.

Facilitators. Factors related to firefighting, other people, personal factors, departmental factors, and system factors were identified by participants as facilitators of motivation.

The most commonly discussed facilitator of motivation were factors related to the job of firefighting. Six of the eight focus groups explained that their performance while on a call can greatly increase their motivation to make healthier choices, particularly when they become easily fatigued or winded on a call. One firefighter described being motivated to make healthy changes after "a call that you go on and you use up three bottles of air and come back and you think, I've 
gotta do something! I am dying here!" Concern about the performance of firefighters next to them also can encourage healthy change. One department decided to implement mandatory physical activity each day because, as one firefighter explained,

Nobody wants to have to save each other, you know. You don't want four guys in shape and one guy not. We don't want to have to not count on that guy, you know. We've all got each other's backs. If I'm going in with him, I want to know he's physically able to drag me out of a fire if he needs to and vice versa. We don't wanna have to be worrying about our brothers falling down on us, you know, not being there, 'cause our lives are in each other's hands essentially.

Similarly, two focus groups specifically discussed feeling fatigued while on a call as motivation to eat healthier or engage in physical activity. Two focus groups also talked about competition as a great motivator for them. Other job related factors that positively influence motivation included gear not fitting, seeing unhealthy people on a call, prioritizing health, caring about health, the physical demands of the job, loving the job, and financial incentive from firefighting. Along with the job of firefighting, other people can also increase motivation. Social support was identified in two of the focus groups as a facilitator of motivation. One participant explained, "If you can't do it, then there's somebody right behind you that can, so we're all brothers and sisters, so we kind of take care of each other." That support can help encourage healthy behavior, knowing that each firefighter always has someone supporting and encouraging them. Outside of the fire department, two focus groups also talked about family support, and how important that can be in facilitating healthy living. Two focus groups mentioned the role of doctors, and that hearing a doctor encourage weight loss or health behavior change can increase the motivation to make said changes. Other ways that people facilitate motivation included 
family responsibilities, and encouragement.

Though personal, departmental, and system factors were also discussed, they were not talked about with as much frequency as the other facilitators. Personal factors that participants mentioned increase motivation included increased energy from healthy living, increased awareness of health concerns, and the importance of helping the community. System factors that can positively influence motivation included insurance benefits and incentive programs. Finally, the departmental factor discussed by two focus groups was limited staff being available at their department. If one firefighter was unable to perform their duties because of health concerns, there are limited active firefighters at their department that can step in and help. One career firefighter explained,

Especially in a small department like this, where I don't have the luxury of riding in the front right seat, which is the officer's seat, once I have 15 years in, and maybe not have to do as strenuous activity. No, if I go out here and I'm by myself, and there's somebody in a house on fire or somebody needs to be moved out of a dangerous situation, that's on me whether I'm 50 or whether I'm 25.

Knowing that staffing is so limited encouraged participants to stay as healthy as possible so they could continue performing their job.

Sleep. According to the participants, being a firefighter has significant negative effects on sleep patterns. All eight of the focus groups talked about their sleep being consistently interrupted throughout the night. Even if they do not travel to a call, should an emergency call come in during their normal sleep hours, they are awakened by their alarm, interrupting their sleep. One volunteer participant explained that in the past he would not go back to sleep after a call, "I've come home and then just took a shower, drank, made my coffee, and went to work. 
Didn't even go back to bed." Career firefighters experience similar disruptions, as one participant explained, "It's very seldom that you may get a two to three hour window of sleep without being woken up in the middle of the night." In the same way, these late night calls also lead to sleep deprivation or a lack of sleep, a topic discussed in six of the focus groups. One volunteer participant admitted that a full eight hours of sleep is not possible, saying "What they're talking about, full night's sleep, you don't get that if you work or volunteer or something like that. You know, it's just not there." Another volunteer firefighter outlined the unhealthy cyclical nature related to a lack of sleep, "It [missing sleep] takes that stress level up again, and that goes back into your eating habits, and it's just a continuing cycle. You miss your sleep, you don't eat right, and you're just on a downhill path there." Unfortunately, even when firefighters are able to sleep, two focus groups mentioned that the quality of sleep is not good. One focus group mentioned that these sleep disruptions can also impact the sleep patterns of their family members, like their children or spouses.

A number of barriers play a part in negatively impacting the sleep of firefighters. Job related factors beyond the calls themselves can negatively impact sleep. Three focus groups talked about the role of adrenaline in disrupting sleep. When a call comes in, they feel a rush of adrenaline, which does not start fading until after a call is complete, and even then the participants reported that it takes time to return to baseline. Therefore, they are not always able to finish a call and immediately go back to sleep. One participant explained, "If I get up in the middle of the night and go on something after I've been asleep, I'm so pumped up from being on whatever we've had I can't go back to sleep. So I'm usually up for the rest of the night." Also, there is a certain anticipation of calls, a factor mentioned by three focus groups. Concern about missing a call, not hearing an alarm, or wondering when the next call will come in keep 
firefighters up throughout the night. One career firefighter explained, "You're just waiting, just waiting for the tone. And you're like, oh, no, it's nothing, roll back over, try to sleep again." Another career firefighter echoed the same struggle, "You try to get to sleep, but you don't wanna sleep through a call so you do sleep on edge and every little sound you hear you're up and you're thinking did I just miss the page? Do I need to get up?" Once a call is over, if the firefighter is able to go back to sleep, two focus groups explained that sleep often does not come because they ruminate about the call they were just on. One participant explained,

Certain calls you go on, you come back home, you know, you think did I do everything I could have done? And you know, that keeps you up. You go on a bad car wreck, did we get the patient out fast enough, did we get them to the hospital. Um, was there a better way to put that fire out? And you start processing all this. Should I have went in this door, should I have went in that? And you try to stop thinking about it 'cause you're so tired and you know you have to get up and be at work at 7:00 AM the next morning. Other barriers to sleep include personal factors such as age and choosing not to sleep, and other people such as not wanting to wake family members by returning home.

Participants recognized that their disrupted sleep patterns had a negative effect on their health. Three focus groups admitted that the disrupted sleep or lack of sleep led to significant fatigue and disorientation. One participant explained, "I am tired the next morning. I'm dragging!" Another participant expressed similar struggles, "If I'm up and we've been on a car wreck for two or three hours, then like I said, I'm done for the night. I'm grouchy and I just want to lay around. I'm tired." Other effects of inconsistent sleep identified by participants included lack of recovery, loss of attention, illness, and less patience.

To balance the negative effects of the inconsistent sleeping habits, participants mentioned 
a number of coping strategies they use. Coffee and caffeine use was discussed by one focus group. Other coping strategies related to becoming immune to the lack of sleep, or adapting to the disrupted sleep as much as possible. One focus group talked about the process of getting more comfortable sleeping at the department to try and improve the quality of sleep when they are able to sleep. Regardless of the coping strategies used, one focus group discussed the importance of recognizing that each individual firefighter is going to have unique strategies to overcome their poor sleeping habits.

Stress. It is not surprising that firefighters experience stress in their daily lives. Throughout the focus groups, participants discussed multiple sources of stress that they encounter, as well as coping strategies they use to overcome that stress. Regardless of the coping strategies used, participants also discussed the negative effects of stress on their health.

Sources. The two most frequently discussed sources of stress were going on calls, and general aspects of firefighting. When talking about calls, three focus groups mentioned the difficult things that firefighters see when on a call, and how those sights and sounds can stay with them, causing them stress not only on the call, but for years after. One participant simply stated, "We've all seen things we shouldn't see." Another participant elaborated further, I've heard some people that's been firemen for 10, 15, 20, 30 years say you know, like, there's that one call that's gonna stay with them the rest of their life. It's not just gonna be a period of time, it's forever. I mean, that's something they're gonna think about, and it is, it's a big deal.

Additionally, in more rural areas, three focus groups expressed concern when a call came in because there was a high likelihood that they would know the victim of the emergency. The pressure to save someone is already high enough as a firefighter, but when the victim is a 
personal friend, co-worker, or family member, participants reported that the stress becomes exponentially higher. A career firefighter in a rural area explained,

We're such a small community, we know everybody ... When we're sitting up here and tones drop, all of our hearts stop 'cause we're like who's that gonna be? Is that gonna be my loved one, is that gonna be somebody I hang out with everyday or work with? And we're going to their house to you know, go pick them up or is their house on fire. It's tough sometimes, especially when you have to work on people that you work with everyday, or you know, love, and you gotta go pick them up. Makes it a little difficult to deal with.

Another source of stress discussed in three focus groups was the uncertainty of the call. When a call comes in, firefighters receive a general description of the emergency; however, participants explained that many times they will reach the site of the call and it will be far worse than originally described. Therefore, traveling to a call and wondering what they will be facing can cause stress. Similarly, two focus groups elaborated that certain types of calls can cause more stress than others. One focus group mentioned that fires can be a bit less stressful, but a heart attack or medical emergency, particularly if it is someone the firefighter knows, can be far more stressful. The responsibilities of their job also cause stress, a source of stress discussed in a few different ways by three focus groups. Some groups discussed the responsibilities of the job in general, another specifically focused on the responsibility of saving people, and another talked about the responsibility of being the end of the line, not being able to leave the site until a solution is reached. Other sources of stress from calls included anticipating calls, and multiple job demands on a call.

Beyond calls, participants explained the stress of firefighting in general. Half of the 
focus groups explained the stress related to the uncertainty of their schedule. One firefighter explained,

You could be going from resting, or doing a training class or whatever and going right out to hard work, you know from being fairly relaxed, to your adrenaline pumping and doing everything you have to do to save life and property ... And the call volume expecting that you run, you can go six months without seeing a fire and then you can go two back to back.

Further, firefighters never know what type of call it will be, whom they will be going to save, or how long the call will take to resolve. All of this uncertainty can cause a great amount of stress. Along with the uncertain schedules, two focus groups mentioned the physicality of firefighting as a source of stress. Another two focus groups talked about the adrenaline experienced when the alarm goes off as a source of stress. As soon as the firefighters hear the alarm, they immediately feel a physical reaction. One participant eloquently explained that when the pager goes off, "you're from zero to 70 instantly," which participants felt leads to increased stress. Other sources of stress from firefighting included job responsibilities, administrative stress, the alarm, and being overworked.

Also within firefighting, focus groups identified stressors that arise from the department. Two focus groups mentioned limited staffing as a source of stress. One participant explained the stress that limited staffing can place on firefighters while on the scene of an emergency, In other places when you've got 40 people in a department, that's, you know, that's an option for them [taking a break after going through one bottle of air]. They go through their bottles and they're done, but we, we don't have that option. We've got to keep going. 
Another participant in a different focus group explained the added stress on smaller departments compared to those in more metropolitan cities,

... You see, like New York, they have a house fire, they probably have 70 guys, where we only have, today we're fortunate and we have 12 on duty. A month from now we'll have 10 every day. So we have a house fire, we have 10 guys and that's it.

From an administrative side, one focus group explained that departments can also cause stress because of budgetary concerns, either from limited community donations or high costs such as those for workman's compensation.

People outside of the fire department can also be a source of stress for firefighters. Two focus groups brought up the public as a source of stress. One participant explained that the public can present mixed messages to firefighters which causes stress, "We get stress from the people too, because if we're going on an emergency, not to their house, we go too fast. But if we're going to their house we can't get there fast enough. And I mean it's a constant battle." Another participant described the pressure from the public while on a call, and the stress that comes from being watched so closely,

The public, you know, wants to see you at your best, you know, and whether you're feeling good that day or stressed out or whatever, you sort of have to put up, put your smile on or whatever you need to do when you're out in the public because they're looking at you all the time.

Other ways that people can cause stress for firefighters included the younger generation and the lack of work ethic, and interpersonal stress from interacting with the other firefighters at the department. Sources of stress not discussed as frequently included stress from the environment in the form of a stressful society and the economy, and stress from other jobs besides 
firefighting.

Despite the source of the stress, participants expressed that the stress experienced as a firefighter is often both mental and emotional, more than physical. One participant described the emotional stress as a rollercoaster, saying "You just know something's gonna happen, and it's, it's a rollercoaster basically is what it is. I mean, you're just, your emotions are up, and then they're down, and it takes a lot out of you." Another participant explained the mental stress, There's always those certain calls that stick with you. Anything with kids bothers a lot of people. Anytime you lose somebody in a fire, that bothers a lot of people. I mean, I've dealt with both those situations myself and it's not, it's not always the everyday stress, it's not the every shift stress your career. It's, most of the time the stress that is most detrimental to us is the one we get every six months or once every year, maybe once every 10 years if you're lucky. I mean, that's the one that I feel effects us mentally more than anything.

Regardless of whether the stress is mental or emotional, another focus group mentioned that if a firefighter is unable to cope with stress, it can lead to an inability to perform their job.

Coping. While many focus groups discussed similar sources of stress, participants outlined a number of different strategies used to cope with stress. Perhaps the most commonly discussed category of coping, however, was coping at the departmental level. Half of the focus groups described debriefing that takes place at the department after a call, providing firefighters an opportunity to vent about what they saw, what happened, and how to move forward. One participant explained that these debriefs are important because it provides an opportunity to voice an opinion,

Whatever you feel that, you know, you can voice your opinion and that let, that helps get 
rid of some of the stress, because we're all, we've all been there. I mean, most of us have been there ... And if we can just talk to each other about it ... We just more or less talk among ourselves.

Another participant explained the importance of these debriefs before going home, as they provide an opportunity to try and relax,

I think by sitting, coming back here and staying here and talking for a while, whether we even talk about the call or not, let's say it wasn't that stressful of a call, it just gets everybody in a different mindset when they're going home that they don't think about the call, they're thinking about going home and going to bed or going to work or you know, whatever it might be. I think just the talking in general makes it easier to relax.

Another powerful form of coping at the department that was talked about in three of the focus groups was joking. Laughter provides a way for firefighters to release the stress that they experience while on a call, though they understand that the public may not appreciate this form of coping or see it as acceptable. One participant explained, In a way you might think firemen have a sick sense of humor, you know, but we have to do it to keep ourselves sane because if we didn't joke about some of the things we saw and just you know, kept it in, you'd see a lot more firemen with a lot more psychological problems. I mean, you have to come back and you have to joke around, because if you don't that stress will really get to you and it will take a toll on you.

Participants later explained that the joking and poking fun is at each other, not the public; however, joking and laughing are reportedly a powerful form of stress relief for firefighters. Other coping strategies at the departmental level included social support, added staff, and trusting other's instincts. 
Another commonly discussed method of coping with stress was through different activities outside of firefighting, or getting away from firefighting in general. Some different activities used by participants for stress management included exercising, snowboarding, and skiing. Two focus groups emphasized the importance of being away from the department as a method of coping. One participant said, "You've gotta step away from this place from time to time ...You've gotta break it up." Another participant echoed the same idea, explaining, "I think just taking a couple days off, away from it. You know, going out and doing something." Regardless of what the firefighter does away from the department, participants expressed the need for time off to recover from the stress. Other forms of getting away from firefighting included escaping at home and having a different second job.

Personal coping strategies were also talked about in the focus groups. Two focus groups explained the importance of finding the individual coping strategy that works for each firefighter. Additionally, two focus groups mentioned a specific personality required to be a successful firefighter, a personality that is able to buffer stress better than others. One participant elaborated,

In my opinion, it takes a certain type of person to do this job. I mean, it is not for everybody. You gotta be able to handle stress well, and be able to make decisions, you know, be able to find ways to cope with that too, cause if you know, normal, I don't want to say normal people, but it can wear people down, and I mean you know, show the effects on a lot of people. Like pretty much everybody up here's got a pretty strong emotional side to them. You know, we don't let much beat us down, our whole crew is strong.

Firefighters in one focus group also discussed their faith as a means of coping, and ignoring the 
stress.

Mental coping strategies were another way firefighters managed their stress. Two of the focus groups outlined the importance of trusting their instincts, particularly while on a call. One participant in a rural department described,

We have to make decisions and go with them too. Like where we're alone a lot of the times, like, you gotta trust your instincts and go with it. If it doesn't work, then you gotta step back, evaluate and punt again. You don't have time, being by yourself, you don't have time to second guess and be like, uh, and look for somebody like, well, is this gonna work?

Other mental coping strategies included not ruminating on calls, not festering, adapting to the stress, and getting closure about calls.

Unexpectedly, some participants use firefighting as a method of coping with stress. Participants discussed the physicality of firefighting, wanting to go on calls, the excitement of the job, and volunteer firefighting on the side as forms of stress management. A more expected stress management technique was using food or drink to cope with stress. Two focus groups discussed their reliance on coffee, and two focus groups also mentioned using alcohol to cope with stress. Other food and drink strategies included group meals and using chew.

Effects of Stress. Similar to sleep, firefighters acknowledge that the amount of stress they experience negatively impacts their health. One focus group felt that the stress weakens their immune system. Another affect identified by a focus group was forgetfulness. A participant in one focus group mentioned that he feels significantly older than his actual age because of the stress he experiences. Overall, one focus group explained that regardless of the exact effects, many firefighters find that the effects of the stress often are not fully experienced 
until after retirement.

Potential problems from obesity. Beyond the factors discussed above, firefighters discussed potential problems on the job that can arise when a firefighter is obese. Such problems included a firefighter being a danger to themselves (e.g., potential injury, stress on the heart from adrenaline), a danger to others (e.g., injuring others, endangering others), or basic firefighting concerns (e.g., inability to perform the duties of the job, breathing on scene, and cost of new equipment if old equipment no longer fits). It is unclear, however, if awareness of these potential concerns leads to changes in each firefighter's motivation to be healthier.

Potential solutions. Firefighters across the eight focus groups agreed that health and obesity are problems in the fire service; however, less agreement was reached in discussing potential solutions. One focus group even discussed the idea that there really are no changes that could be made that would benefit the health of firefighters in West Virginia. However, other firefighters discussed four potential options: certain changes or interventions, incentive programs, education, and physical requirements.

Potential interventions. A variety of changes or interventions were discussed among different focus groups, including options related to age, changing others, changing the access and availability of healthy options, changing requirements, and personal changes. While discussing age, firefighters mentioned recruiting younger firefighters, or creating a "youth pill" to help older firefighters, as they believe that younger firefighters tend to be healthier. Building social support among firefighters in the department and developing accountability were changes that could be made to surrounding people that might help. Many of the changes to access and availability were related to food, such as limiting unhealthy options, providing coupons for produce, offering healthy food donations, making healthy foods available, or bringing in chefs for the departments. 
Beyond nutrition, one focus group discussed making facility improvements to increase the availability of opportunities for physical activity. Within focus groups, firefighters brought up the option of creating different requirements, such as yearly physicals with a doctor, mandated breaks or meal times, or even smoking and drinking cessation programs. When these changes were brought up though, there was disagreement about their potential effectiveness if such changes were mandatory. Two focus groups each discussed the importance of making personal changes, such as making changes to the individual's diet and exercise routines, or changing an individual's priorities or mindset to emphasize health and wellbeing. Unfortunately, further ideas for how to change routines and mindset were not discussed. Despite the multitude of ideas, one participant mentioned the importance of a comprehensive approach, mentioning that no intervention will be effective unless it approaches the problem of health and obesity from multiple angles.

Interventions. Along with potential changes, firefighters discussed different interventions that could be created to encourage and motivate a healthier culture. First, departmental incentives were considered. One focus group said that offering equipment to the department as incentive for showing health improvements would continue motivating them to be healthier. Two other focus groups thought having a budgetary incentive would help; offering the department money for showing health improvements of their firefighters. Beyond the department, firefighters also talked about personal incentives. Two focus groups discussed about the power of a financial incentive, or offering a bonus structure to firefighters that are able to maintain a certain level of health each year. Finally, incentives at broader, systematic levels, were brought up. Again, two focus groups said an incentive from insurance companies could help; for example, lowering premiums. Legislative incentives were discussed by one focus 
group as another potential option for positive change.

Education. Along with interventions and incentives, five focus groups discussed their openness to education. Free nutrition education was the most popular request, mentioned by four of the eight focus groups. Building awareness of the obesity and health problem within the fire service, and stress management or stress education were each discussed by two focus groups. Other opportunities for education discussed by focus groups included displaying prompts around the fire house to encourage healthy decision making, and exercise education specific to the needs and demands of firefighters.

Physical fitness requirements. The last potential solution, discussed by five focus groups, was creating physical fitness requirements for all firefighters, a requirement that does not currently exist beyond initial recruitment. However, while this solution was mentioned, most firefighters wanted to discuss the barriers to this approach; only two focus groups discussed this change as a viable option. One barrier to implementing a physical fitness requirement was the already limited staff at many departments. One focus group acknowledged that many of their firefighters would likely not pass a physical fitness test, and they would then be forced to find replacements, which they would not be able to afford. A participant explained,

In a volunteer, it [mandatory fitness requirements] would drive, we would lose members and volunteer fire departments are short-staffed now. You can't afford, we can't afford to lose any more members. Anytime we lose a member, that's a guy that's not there to back you up. The fire department invests a lot of money for everybody, with the cost of gear and training, I mean it's not an option. We cannot have a program that would, unfortunately, making them be healthy 'cause we would have to get rid of people. Similarly, one focus echoed that losing members because of mandatory fitness requirements 
could even lead to departments closing,

You go to these little departments out in the sticks and there's guys that are four, five hundred pounds that are firemen ... If they would go and have that physical done, would they pass? Probably not. So you take that little department and you take those couple guys that are overweight, that aren't fit, and what does that leave you with? Probably two or three guys. So you have two or three guys in a small department, what good is that gonna do you? None. It's not gonna be long and they're gonna be shut down.

Further, because of the time demands already put on firefighters through their training; the requirements to become a firefighter are already so great that if further requirements were set (i.e., physical fitness), firefighters are concerned that recruiting would become even more challenging, or that some current volunteers might quit. Two focus groups also talked about negative previous experiences with fitness requirements, and the rebellion that ensued after firefighters tried to meet those standards. The previous requirements that were set were eventually eliminate because people took advantage of disability programs offered after failing fitness requirements.

Career and volunteer perceptions. During the focus groups, participants began discussing the myriad differences between career and volunteer firefighters. Two of the four volunteer focus groups discussed the ease with which career firefighters can exercise because they have equipment available to them at their department. One volunteer participant explained that career firefighters have "equipment and everything in house." Another volunteer expressed, I think truly it's a lot easier for a paid guy to you know [workout]. And there's even some departments that take time outta that, your shift, that you're to be in there and working out. So, I, it's just a lot easier for the paid people to do it than it is a volunteer. 
Overall, the volunteers believe that engaging in physical activity would be significantly easier for career firefighters because of their access to equipment. Further, one volunteer focus group believed that career firefighters were "required to workout so many hours a week." While many of the career firefighters explained that this is untrue, volunteers perceived the physical activity requirement as another reason why career firefighters might be healthier and in better shape than volunteers. Finally, because career firefighters work 24-hour shifts and have access to fitness equipment and can cook together, one volunteer focus group felt that career firefighters work in a healthier environment, which again helps them live healthier lifestyles than those of volunteers.

Career firefighters also had their own perceptions of volunteer firefighters. One concern discussed by career firefighters in one focus group was that volunteers might not be as healthy as career firefighters because they are not required to spend as much time at the department. As one career firefighter explained,

It's hard to gauge them and then gauge us. They don't, I mean, we're here 24-7 where they're there one day a month or something, whatever it may be. Appreciate their service and everything, but I don't know how active they stay, or how active it is for them.

Along with the lack of time at the department, career firefighters fear that volunteers might not be learning how to stay healthy for firefighting. Another career participant described,

In West Virginia you have that all across the state, just tons of volunteer departments and they're made up of good people that just want to help. Have no idea what they're doing, or how to be in shape for what they're doing ...

However, beyond time at the department and training, career firefighters identified that volunteers have more personal responsibility for cooking than themselves, a point that volunteers discussed as well. Rather than being able to rely on other firefighters to help cook healthier 
foods at the department, volunteers are eating a vast majority of their meals at home, away from their fellow firefighters. Therefore, even if some of their peers are eating healthier, that might not translate into other volunteers making healthy change as well because they are not around that healthy behavior.

Career versus volunteer comparisons. Though career and volunteer firefighters perceived great differences between the two cultures, few differences came from the focus group discussions. The most common barriers to health behaviors such as lack of time, cost of healthy foods, stress, balancing multiple roles and jobs, lack of motivation, and disrupted sleep were echoed in both cultures. Additionally, suggestions for potential solutions were similar as well, emphasizing the importance of education and different incentives such as financial or budgetary incentives. However, when the average frequency of topics is compared between the volunteer and career focus groups, some differences in emphasis were present. For example, when discussing general factors that influence health, career firefighters were more likely to discuss generic factors and facilitators, while volunteers talked more about barriers than their career counterparts. Further, career firefighters spoke more about nutrition overall in the focus groups, particularly about facilitators to healthy eating, than volunteer firefighters. Conversely, volunteer firefighters spoke more about barriers to physical activity than the career firefighters. Discussions about stress came up more in focus groups with career firefighters overall, and career firefighters talked more about sources of stress and coping strategies more than volunteer firefighters. Finally, career firefighters, on average, discussed more potential solutions overall than the volunteer firefighters. Even though differences did exist, particularly in the access and availability of fitness equipment that career firefighters have and volunteers do not, many of the same barriers and concerns did arise. 


\section{Photovoice}

Nine participants took part in the Photovoice portion of the study; seven volunteer and two career firefighters. Between the nine participants, a total of 55 pictures were received. The average number of pictures sent per participant was six, with participants sending as few as one, and as many as 12 during the seven-day time frame. Three main themes came out of the photographs and summaries sent: nutrition, physical activity, and other miscellaneous factors.

A total of 27 pictures $(50.0 \%$ of pictures sent) focused on nutrition. Of those 27 pictures, $11(40.7 \%)$ showed the firefighter making a conscious healthy choice with whatever they were eating. For instance, one firefighter sent a picture of a salad and explained, "Taking time to eat a healthy lunch instead of fast food large salad and water." Another firefighter also sent a picture of his meal, and said, "Thursday lunch. This is a particularly health lunch, except for the fact that the chicken salad croissant was twice what you see. I saved the other half for dinner..." Another common theme related to nutrition represented in six pictures was that of time. One firefighter took a picture of an almost empty fridge and shared, "Have not spent much time at home past few weeks ..." After sending a picture of his car, another participant shared, "Yesterday was so stressful on the road from 9:30 am till 11:30 pm not much time to eat good it was McDonalds on the run." Positive motivation, conscious unhealthy choices, negative cues, and food as fuel were each represented by two pictures each. Work, availability, water, negative social pressure, fatigue, and convenience were each represented by one picture each.

Nutrition was another theme represented in 13 of the 54 pictures (24.1\%). While most themes were shown once, a few themes were represented twice. Obligation was represented in two pictures, one from a career firefighter and one from a volunteer. After sending a picture of new running shoes, a career participant said, "Just went and bought these cause you made me 
feel bad about being fat!" Conversely, a volunteer firefighter shared a picture of a snow shovel, explaining it as "forced exercise!" Another theme represented more than once was preferred activity. One participant shared a picture of a Ping Pong table and explained, "One of my favorite ways to be active without really even noticing it." The other picture showed a snowcovered mountain, and said, "Saturday, skiing at [a local] mountain." Two firefighters also each sent a picture representing the positive influence of improving weather on their physical activity. One sent a picture of the five-day forecast available on his phone and explained, "There's nothing more motivating than signs of spring in the forecast!" The other participant sent a picture of the sun through a tree and said, "Getting outside after a long and cold winter and being more active." Other pictures related to physical activity showed sub-themes of incentive, convenience, extrinsic motives, conscious healthy choices, stress management, positive cues, sedentary behavior, negative weather, and time.

The final theme shown in photos sent by participants was of other miscellaneous factors they felt impacted their health, shown in 15 of the 54 pictures sent (27.8\%). The most commonly represented theme was of time, without specific discussion about nutrition or physical activity. One picture was of a clock hanging on a wall, and the participant explained, "Never enough time. Just left the office, fundraising meeting at the fire house in an hour." Another picture representing time was of grapes, a water bottle, and a firefighter's pager, and the participant simply said, "Waiting to go on the next call." Another theme shown more than once was of tobacco use, specifically through a picture of chew ("With it not healthy. Without, eat too much, still not healthy.") and cigarettes ("It's effects are obvious!"). Sleep was represented in the miscellaneous theme as well. One participant sent a picture of one of the trucks used at their department and said, "Early morning call lack of sleep." Similarly, the second picture was also 
of a department truck and explained, "No sleep house fire last night. Up all night, work all day." Stress was the other theme represented twice. For example, one picture showed a woman's hand wearing a wedding ring, and the participant explained, "Stressful not knowing how it will go a lot of thinking depression thinking of the bad things." Other sub-themes represented in pictures and explanations included work, on-the-job safety, improving weather, social obligation, health warnings, convenience, environment, and stress management.

\section{House Tours}

After touring and mapping the eight fire houses that were visited, clear discrepancies appeared. All four career departments had fitness equipment, three of which had designated fitness rooms, and one had a treadmill and a few weights available. While the paid chiefs and firefighters discussed the ease and convenience of having fitness equipment on site, two of the four career departments had the equipment located in an area away from where the firefighters normally "hang out"; therefore, while it was on site, the equipment might not be used as much as expected. However, the two departments with fitness rooms near common areas of the house discussed the frequency with which the rooms are used. Another important point discussed at the large rural and urban career sites was that they have multiple houses throughout one city, so while one house might have a fitness room available, firefighters serving at other houses would have to travel to use the equipment, creating an additional barrier. Further, some of the chiefs and firefighters complained that the equipment was not always working, was not up-to-date, or was not sufficient for the type of exercise they would like to do. Despite the concerns and complaints, many present for the house tours liked the fact that the equipment was there, and often wanted to have more, but their budgets would not allow for it.

None of the volunteer departments had fitness equipment available at the department. 
One chief discussed an upcoming renovation and expansion, which included the addition of a fitness room. Another volunteer chief mentioned the limitations of the building they were in, and because of its size, housing any fitness equipment was not an option as they could barely fit the required firefighting gear. The volunteer firefighters that joined on the tour discussed the interest in having equipment at the department, but understood that space and/or budget restrictions made that difficult. Further, they at times doubted whether or not they would actually use the equipment, even if it was made available to them.

Another unique aspect of the career departments compared to volunteer departments was a common room with a large-screen television and numerous recliners and/or couches. This was present in all four of the career departments, and at times was in front of, or along the path to, the fitness room. One group of firefighters mentioned that the chairs were not as comfortable as they looked, but the other three admitted that the recliners and television created a major temptation to engage in sedentary behavior rather than staying active.

One commonality existed across both career and volunteer fire houses, a large kitchen. The volunteer departments often had industrial kitchens because the house would be used as an event site, or large meals would be cooked for fundraising events. Only one of the career departments had an industrial sized kitchen, but the other three had full kitchens with all of the necessary appliances (stove, oven, refrigerator, microwave) to cook healthy meals. The fact that both career and volunteer departments have access to a full kitchen, regardless of the setting, means that nutrition education could be effective in helping firefighters cook and eat healthier.

Overall, it was impressive to see how well maintained the various equipment and buildings were. Chiefs and firefighters were eager to share the pride they have in their equipment, and admitted that maintenance is key since it is rare that their budgets allow for new 
equipment (trucks, turnout gear, fitness equipment, etc.) to be purchased. However, it was also disheartening to hear how old some of the buildings and equipment were. While some trucks were from the 2000s, a few departments were still using trucks or equipment from the late 1980s or early 1990s, meaning that they were using equipment that was over 20 years old. Even though they kept the equipment in top shape, it was surprising to hear they were forced to use such old equipment because of budgetary constraints. 


\section{Appendix F}

\section{Department Maps}

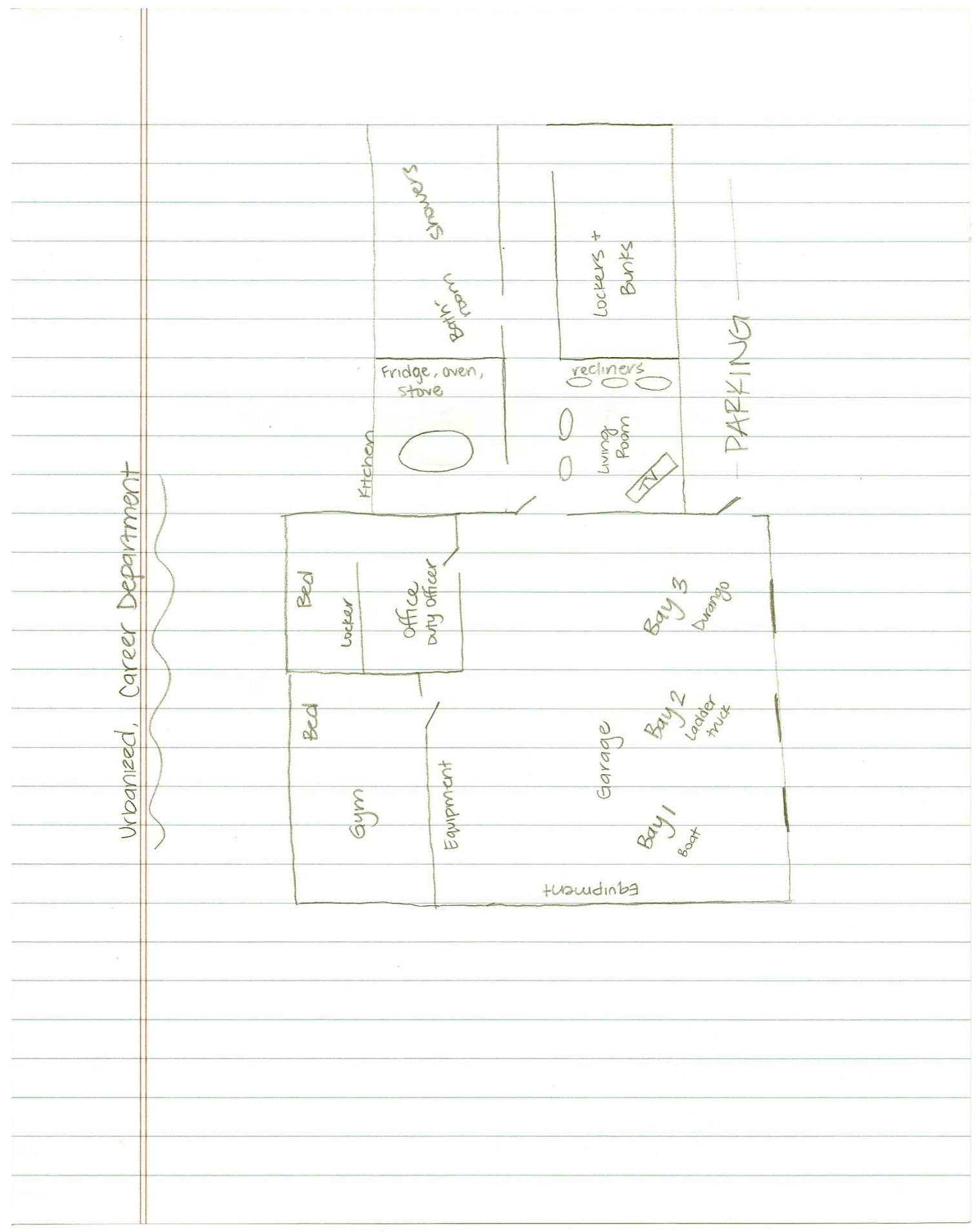




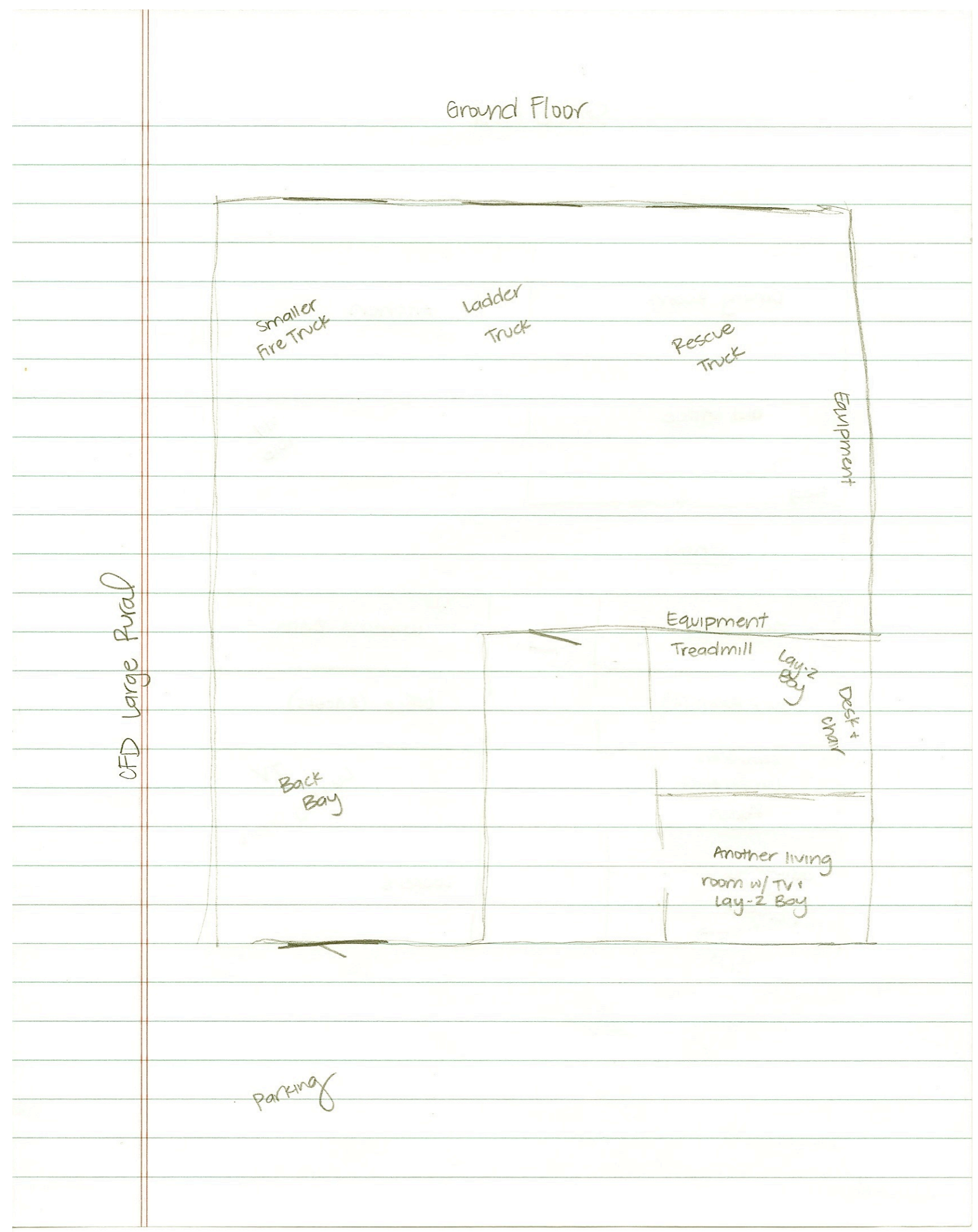




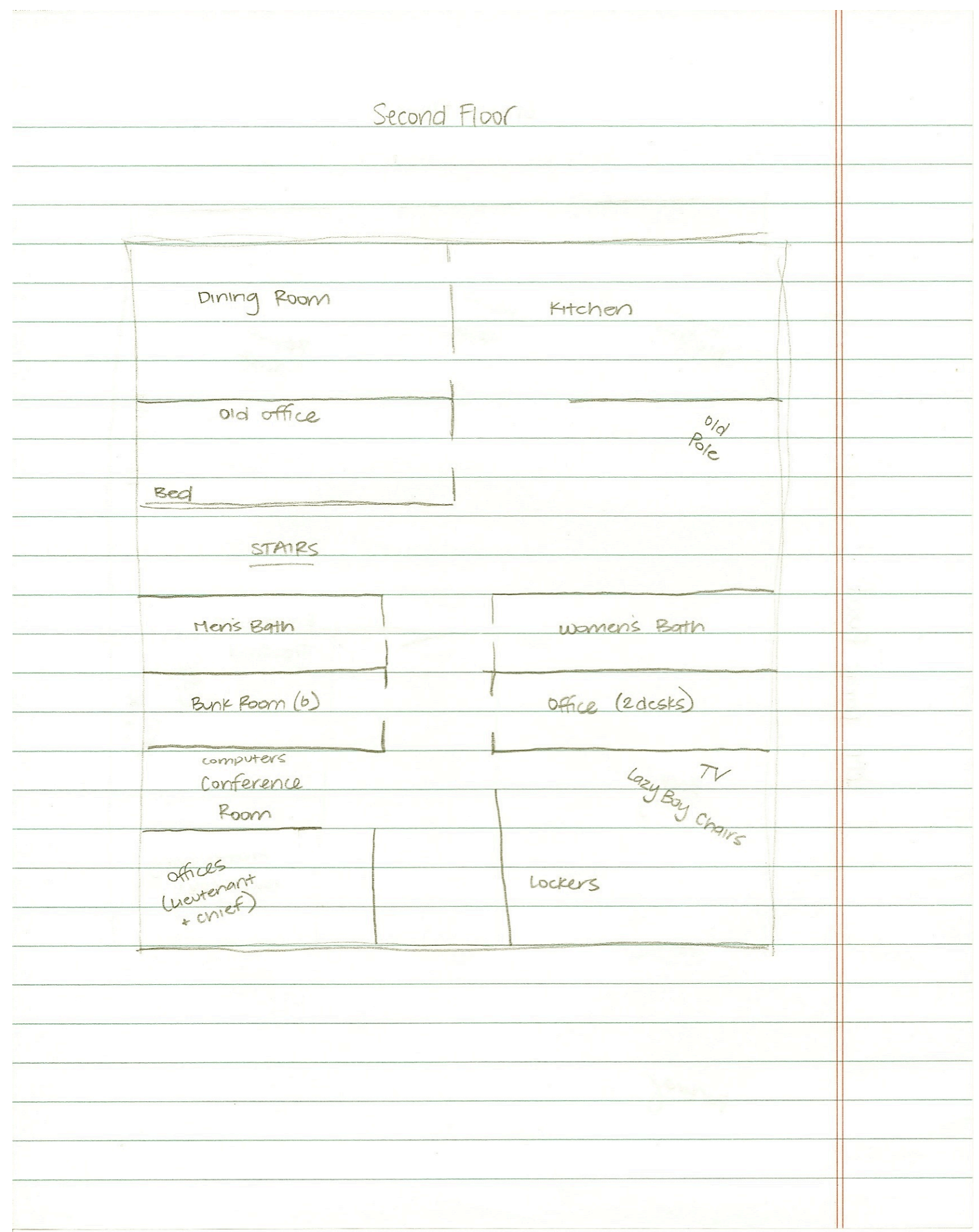




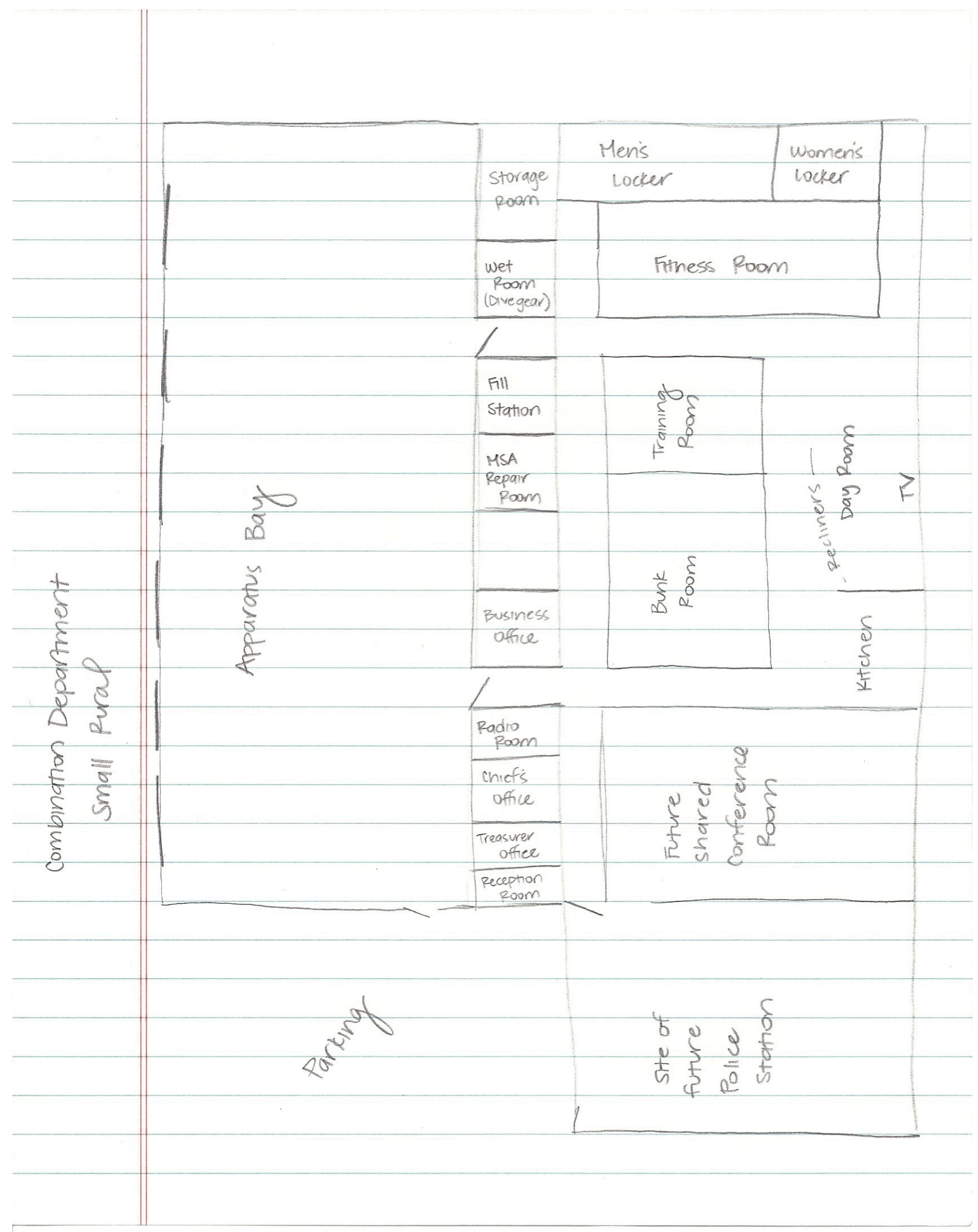




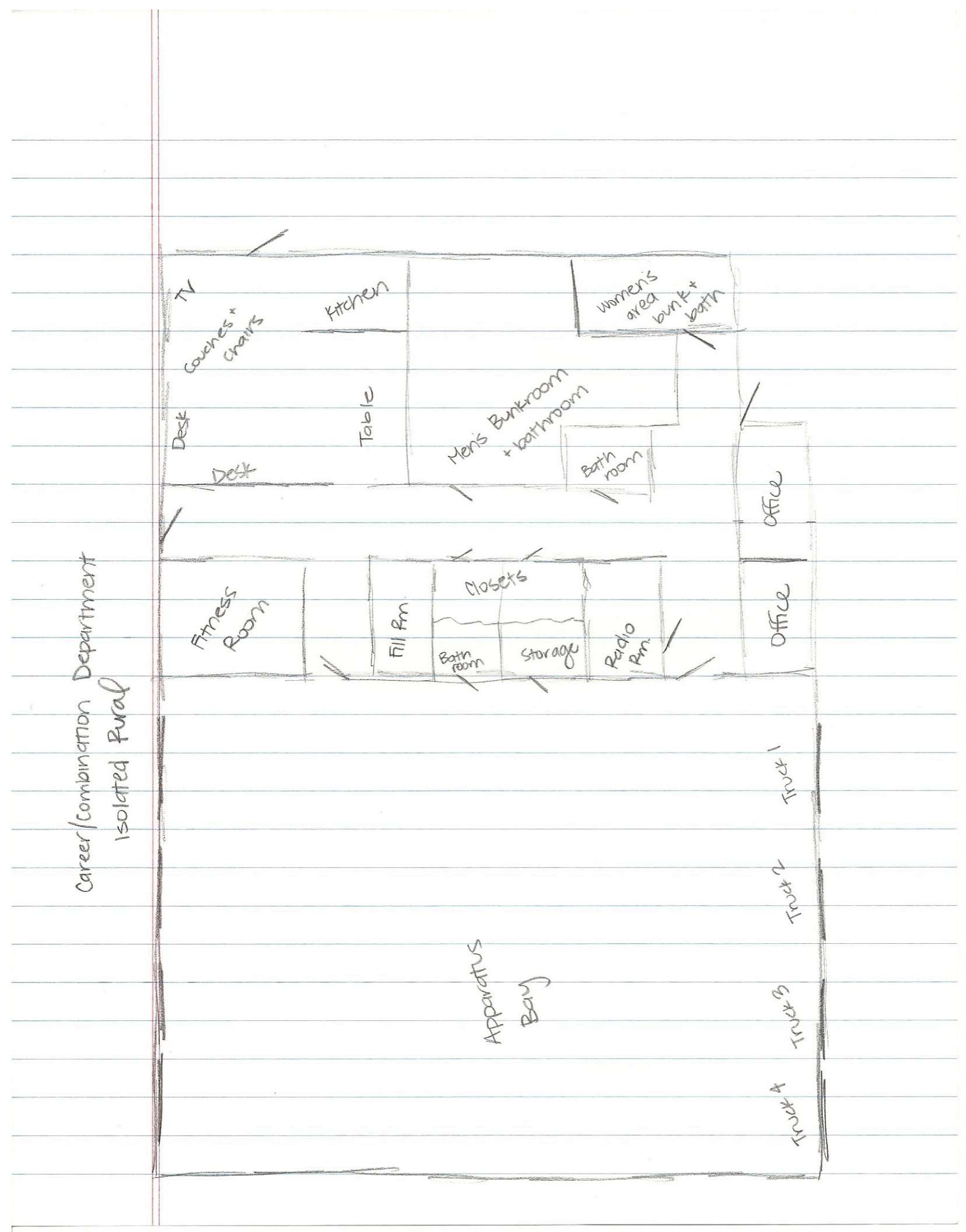




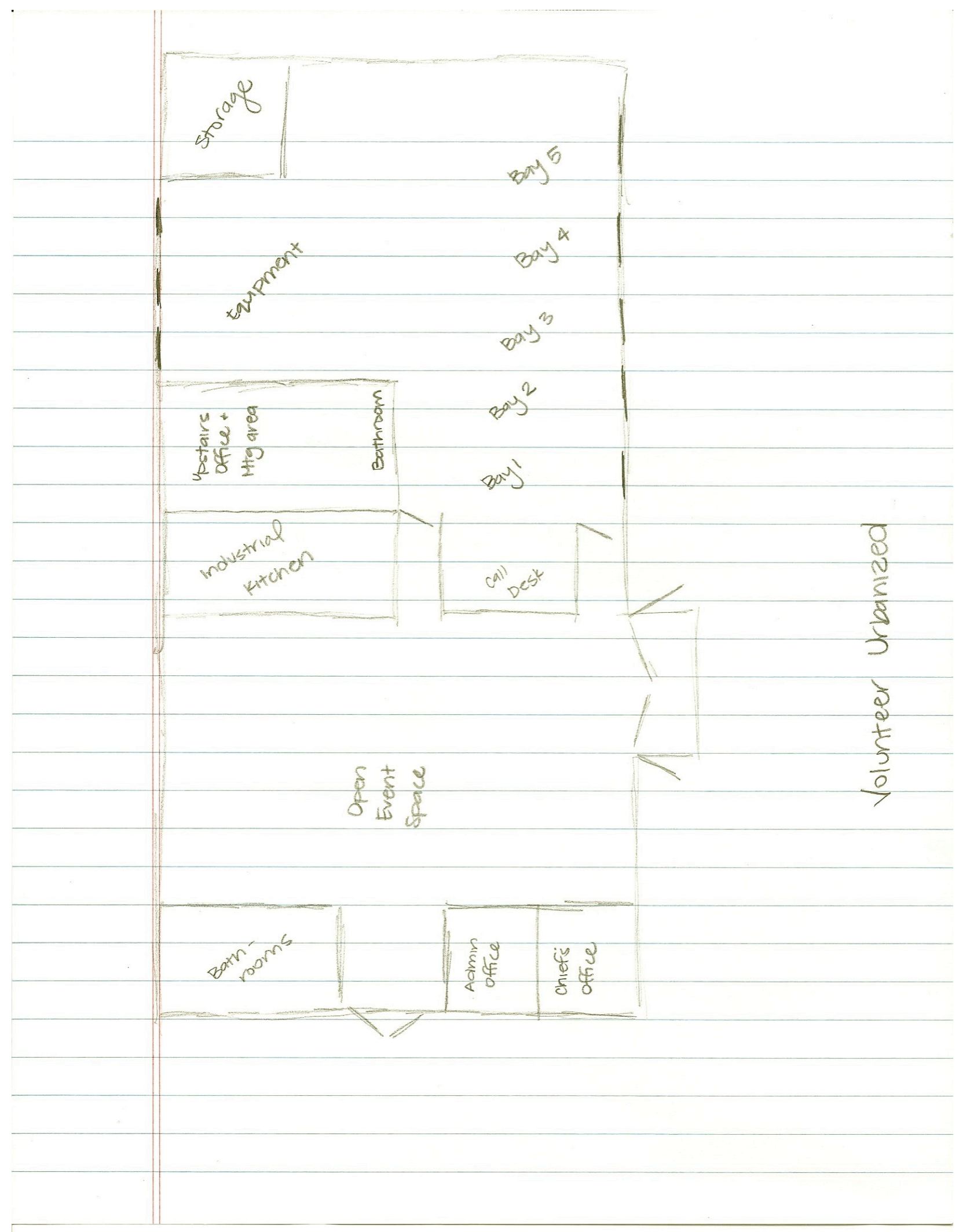




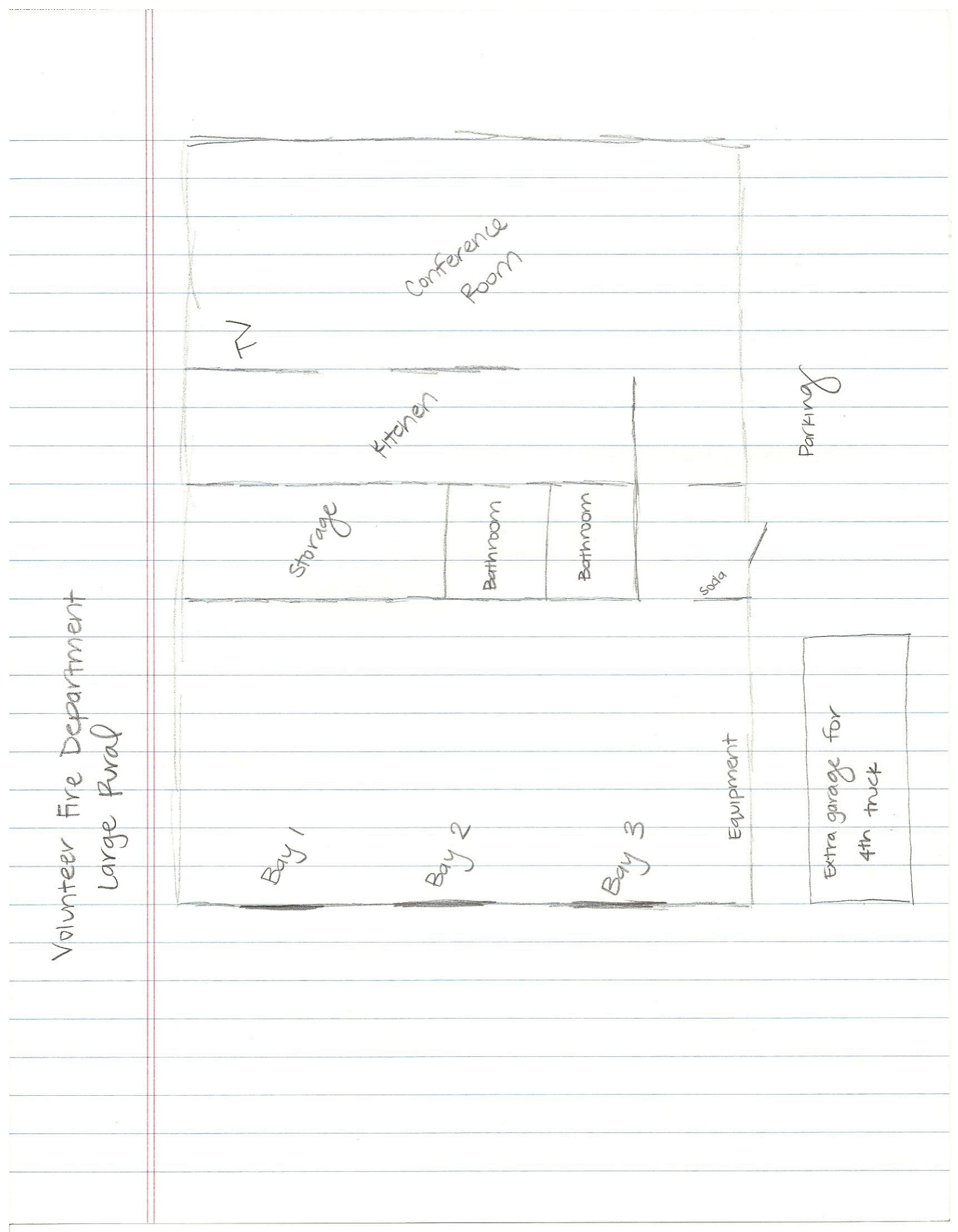




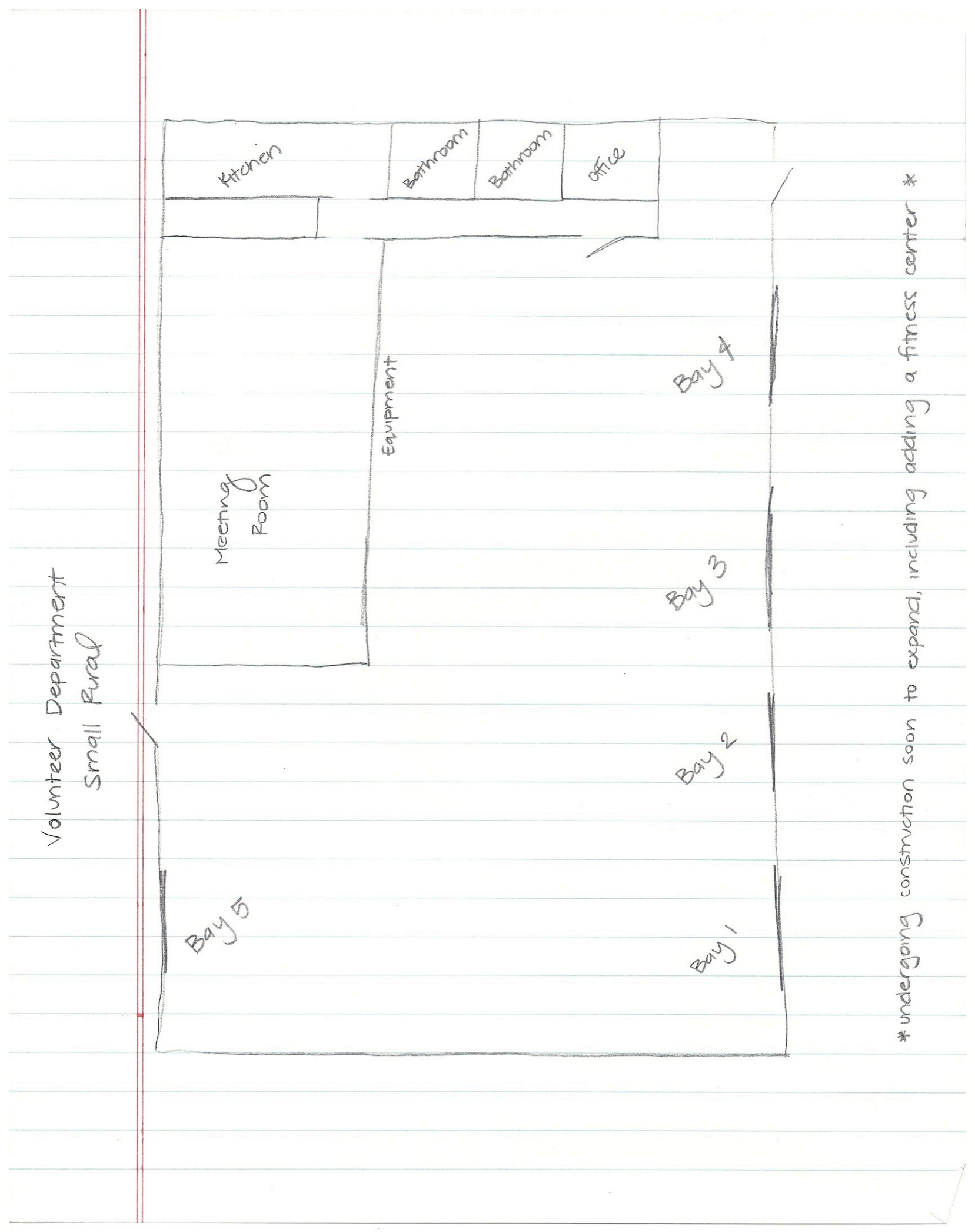




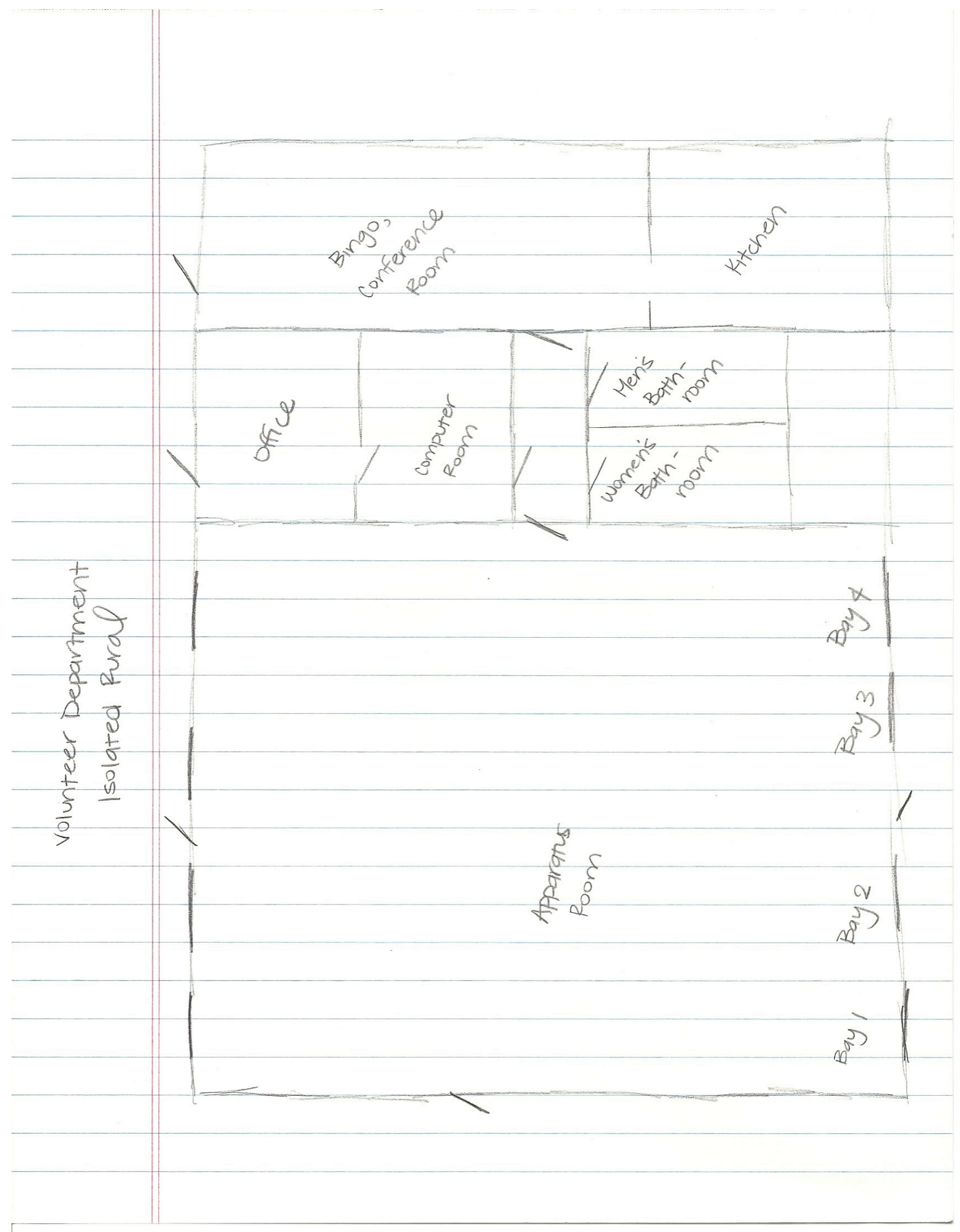

